\title{
The light element abundance distribution in NGC 5128 from planetary nebulae ${ }^{\star, \star \star}$
}

\author{
J. R. Walsh ${ }^{1}$, G. H. Jacoby ${ }^{2}$, R. F. Peletier ${ }^{3}$, and N. A. Walton ${ }^{4}$ \\ ${ }^{1}$ European Southern Observatory, Karl-Schwarzschild-Strasse 2, 85748 Garching, Germany \\ e-mail: jwalsh@eso.org \\ 2 Giant Magellan Telescope Organization/Carnegie Observatories, Pasadena, USA \\ e-mail: gjacoby@gmto.org \\ 3 Kapteyn Astronomical Institute, Postbus 800, 9700 AV Groningen, The Netherlands \\ e-mail: r.f.peletier@astro.rug.nl \\ ${ }^{4}$ Institute of Astronomy, University of Cambridge, Madingley Road, Cambridge CB3 OHA, UK \\ e-mail: naw@ast.cam.ac.uk
}

Received 5 December 2011 / Accepted 30 April 2012

\begin{abstract}
Context. Planetary nebulae in the nearest large elliptical galaxy provide light element abundances difficult or impossible to measure by other means in a stellar system very different from the galaxies in the Local Group.

Aims. The light element abundance pattern from many planetary nebulae (PNe) at a range of radial distances was measured from optical spectroscopy in the elliptical galaxy NGC 5128, which hosts the radio source Centaurus A. The PN abundances, in particular for oxygen, and the PN progenitor properties are related to the galaxy stellar properties.

Methods. PNe in NGC 5128 covering the upper 4 mag of the luminosity function were selected from a catalogue. VLT FORS1 multi-slit spectra in blue and red ranges were obtained over three fields at 3,9 and $15^{\prime}$ projected radii $(4,8$ and $17 \mathrm{kpc}$, for an adopted distance of $3.8 \mathrm{Mpc}$ ) and spectra were extracted for $51 \mathrm{PNe}$.

Accurate electron temperature and density diagnostics are usually required for abundance determination, but were not available for most of the PNe. Cloudy photoionization models were run to match the spectra by a spherical, constant density nebula ionized by a black body central star. He, N, O and Ne abundances with respect to $\mathrm{H}$ were determined and, for brighter PN, S and Ar; central star luminosities and temperatures are also derived.

Results. Emission line ratios for the $51 \mathrm{PNe}$ are entirely typical of PN such as in the Milky Way. The temperature sensitive [O III]4363 A line was weakly detected in $10 \mathrm{PNe}$, both [O II] and [O III] lines were detected in $30 \mathrm{PNe}$, and only the bright [O III]5007 ̊ line was detected in 7 PN. For 40 PNe with Cloudy models, from the upper 2 mag of the [O III] luminosity function, the most reliably estimated element, oxygen, has a mean $12+\log (\mathrm{O} / \mathrm{H})$ of 8.52 with a narrow distribution. No obvious radial gradient is apparent in $\mathrm{O} / \mathrm{H}$ over a range 2-20 kpc. Comparison of the PN abundances with the stellar population, from the spectra of the integrated stellar light on the multi-slits and existing photometric studies, suggests an average metallicity of $[\mathrm{Fe} / \mathrm{H}]=-0.4$ and $[\mathrm{O} / \mathrm{Fe}]=$ 0.25 .

Conclusions. The masses of the PN central stars in NGC 5128 deduced from model tracks imply an epoch of formation even more recent than found for the minority young population from colour magnitude studies. The PN may belong to the young tail of a recent, minor, star formation episode or derive from other evolutionary channels, perhaps involving binary stars.
\end{abstract}

Key words. galaxies: elliptical and lenticular, cD - galaxies: individual: NGC 5128 - galaxies: abundances planetary nebulae: general

\section{Introduction}

Planetary nebulae provide a multi-facetted probe of the galactic environment. As a short-lived product of the evolution of low mass stars, they sample the bulk, by both number and mass, of the stellar population of galaxies of all Hubble types. Their size makes them unresolved from the ground at distances greater than $\sim 1 \mathrm{Mpc}$ but their strong emission lines makes them easy targets for observational work, even when observed against the high stellar background of a galaxy bulge. These advantages have been exploited in a number of distinct areas. Statistics of

^ Based on observations collected at the European Organisation for Astronomical Research in the Southern Hemisphere, Chile in observing proposals 64.N-0219, 66.B-0134, 67.B-0111 and 71.B-0134.

$\star \star$ Tables 5,6 and 8-12 are available in electronic form at http://www. aanda.org planetary nebulae $(\mathrm{PNe})$ in galaxies of various types show a late time indicator of the star formation rate through the luminosity specific PN frequency $\phi$ (Ciardullo et al. 1989). The PN luminosity function (PNLF), despite theoretical difficulties, still proves to be a powerful distance method (Jacoby et al. 1992; Ciardullo et al. 2002). The radial velocity, simply measured from one emission line, provides a kinematic test particle for study of the galaxy potential and in intra-cluster gas of the cluster potential and turbulence (Arnaboldi et al. 2003). The abundances of a number of light elements can be measured from emission line ratios in PN spectra, such as $\mathrm{He}$ and $\mathrm{N}$ and, in particular, the $\alpha$-elements $\mathrm{O}, \mathrm{Ne}, \mathrm{S}$ and $\mathrm{Ar}$, which are difficult to study in all but high resolution and high signal-to-noise spectra of individual stars.

As the closest example of a large early-type galaxy, NGC 5128 (Hubble type S0p) occupies a central place in 
studies of resolved stellar populations in a galaxy different to the Milky Way and M31. This large elliptical galaxy in the Centaurus group shows signs of major activity with an active nucleus, Faranoff-Riley Class I radio lobes, presence of dust and young stars in its inner region (Graham 1979), a young ( 0.3 Gyr) tidal stream (Peng et al. 2002) and stellar shells in its outer regions (Malin et al. 1983); see Israel (1998) for an earlier review. This activity can be attributed to a minor merger which has had little influence on the bulk of the older passively evolving population (Woodley 2006), making NGC 5128 a nearby exemplar of more distant massive early-type galaxies; Harris (2010) provides a recent review of the underlying galaxy properties. The spectroscopy of globular clusters by Beasley et al. (2008) indicates typical ages of 7-8 Gyr from stellar population fitting, reinforcing the presence of a large-scale intermediate age star formation episode. Fits to deep colour-magnitude diagrams also provide evidence for a minority, much younger, population of age 2-4 Gyr (Rejkuba et al. 2011).

As representative of the low mass stars, planetary nebulae in NGC 5128 have been catalogued over a period of two decades, beginning with the catalogue of Hui et al. (1993a). 785 PN were discovered by [O III]5007 $\AA$ emission line and off-band imaging; from the PN [O III] magnitudes Hui et al. (1993b) determined a PNLF distance of $3.5 \mathrm{Mpc}$. Independent measurements from the Mira period-luminosity relation and the luminosity of the tip of the red giant branch (Rejkuba et al. 2005), surface brightness fluctuations (Tonry et al. 2001), globular cluster luminosity function (Harris et al. 1988) and 42 classical Cepheid variables (Ferrarese et al. 2007) result in distance estimates in the range 3.4 to $4.1 \mathrm{Mpc}$. Harris et al. (2010) present a comprehensive review of distance estimates to NGC 5128, and recommend a best-estimate of $3.8 \mathrm{Mpc}$, which is adopted here (distance modulus $27.90 \mathrm{mag}$ ). Peng et al. (2004) extended the original catalogue of PN and found a further 356 PN by filter imaging; 780 out of a total of 1141 were spectroscopically confirmed. Further emission line mapping and follow-up intermediate dispersion spectroscopy has extended this list to over 1200 confirmed PN (Rejkuba \& Walsh 2006). This number of PN makes NGC 5128 a rich source for statistical extra-galactic PN studies, rivalled only by the Milky Way and M 31 (Merrett et al. 2006).

Since there are both a large number of PN and they have been catalogued to large galactocentric distances (to $80 \mathrm{kpc}$ along the major axis by Peng et al. 2004), the radial velocities allow the dynamics of the halo mass distribution to be studied. Hui et al. (1995) measured [O III] radial velocities for 431 out of their 785 catalogued PN. The offset of the rotation axis from the minor axis was attributed to evidence of triaxiality; fitting the rotation curve and velocity dispersion revealed a radially increasing mass-to-light ratio and hence the presence of a dark matter halo. Peng et al. (2004) extended the PN kinematic work to $780 \mathrm{PN}$ and showed the large rotation along the major axis but with a pronounced zero-velocity twist produced by the triaxialprolate mass distribution. The kinematics of the large population of globular clusters (with 563 available radial velocities Woodley et al. 2010, 2007, 2008) show similar kinematics to the PN but with small differences, such as lower rotation amplitude (Woodley et al. 2007).

The NGC 5128 globular clusters (GCs) also provide fundamental evidence of the star formation history. Of the 605 confirmed GCs in NGC 5128 (Woodley et al. 2007, 2010), more than half have metallicity measurements and they divide roughly half and half into metal rich and metal poor above and below $[\mathrm{Fe} / \mathrm{H}]=-1.0$. The metal poor GCs $([\mathrm{Z} / \mathrm{H}] \sim-1.3)$ are older with ages similar to Milky Way GCs and the metal rich GCs (Peng et al. 2004; Beasley et al. 2008) have intermediate ages (4-8 Gyr) and metallicity $([\mathrm{Z} / \mathrm{H}] \sim-0.5)$. There are many more GC candidates (e.g. Harris et al. 2004) and the total number of GCs has been estimated at around 1300 (Harris 2010).

NGC 5128 is close enough that individual stars can be resolved with HST imaging (and from the ground with adaptive optics in the near-IR) and several studies have been obtained outside of the bright bulge where crowding is lower. Soria et al. (1996) detected the red giant branch (RGB) and the asymptotic giant branch (AGB) from intermediate age stars. The same field was followed up with NICMOS photometry and the IR colour magnitude diagram shows stars well above the tip of the red giant branch for old stars, confirming the presence of intermediate age stars (Marleau et al. 2000). Colour magnitude diagrams from HST WFPC2 were constructed for two halo fields at projected radii of 19 and $29 \mathrm{kpc}$ (Harris et al. 1999, 2000). The colour magnitude diagrams are dominated by old stars. Under the assumption that the ages of the stars in NGC 5128 are similar to those of old globular clusters in our Galaxy, the mean metallicity of the two fields was found to be similar at $[\mathrm{Fe} / \mathrm{H}] \sim-0.4$ with about one third of the stars in a metal poor component and the rest metal rich. The metallicities in the halo fields were compared by Harris et al. (2002) to the metallity distribution function (MDF) in a field at $7.4 \mathrm{kpc}$, containing a mixture of outer bulge and inner halo. A broad MDF was found as in the halo fields, peaking at $[\mathrm{Fe} / \mathrm{H}] \sim-0.4$; but subtracting the MDF of the halo fields reveals a peak at slightly higher metallicity $(\sim-0.2)$. Much deeper ACS imaging by Rejkuba et al. (2005) in a halo field at $36 \mathrm{kpc}$ reached to the horizontal branch (core helium burning population) as well as showing the red giant branch, red clump and AGB bump. Again a broad MDF was found with an average metallicity of -0.64 , but with a broad tail to higher metallicity $(>0)$. The age sensitive indicators imply an average age for the halo of $8_{-4}^{+3}$ Gyr. Comparison of the MDF in the four fields shows the peak shifting to lower metallicity with increasing projected radius but the distribution is similarly wide at all radii.

In order to determine light element abundances of PN in NGC 5128 fairly high signal-to-noise spectroscopy is required. Close to the inner bulge the stellar continuum surface brightness is too high and only the strongest few lines can be measured. However in the outer regions by selecting bright PN, diagnostic lines of $\mathrm{He}, \mathrm{Ne}, \mathrm{Ar}$ and $\mathrm{S}$ as well as the brighter lines of $\mathrm{O}$ and $\mathrm{N}$ can be measured and abundances derived. Walsh et al. (1999) performed deep ESO $3.6 \mathrm{~m}$ long slit spectra of a few selected $\mathrm{PN}$ with a long slit spectrograph and measured lines in addition to the brightest line ([O III]5007 $\AA$ ) in five $\mathrm{PN}$. O/H could be determined in two $\mathrm{PN}$ and values of $12+\log (\mathrm{O} / \mathrm{H}) \sim 8.5$ (i.e. $[\mathrm{O} / \mathrm{H}]-0.2$, adopting the Solar oxygen abundance of 8.69 from Scott et al. 2009) were derived. This initial work has been expanded to deeper spectra of many more PNe with the ESO VLT and FORS1 instrument in multi-slit mode. In Sect. 2 the observations are described and the reduction of the spectra and derivation of the abundances are presented in Sect. 3. The results for the PN line fluxes and derived abundances both for the individual PN and for summations of many spectra are presented in Sect. 4. Since the diagnostic lines for electron temperature and density, important for abundance determination, are weak or undetected, photoionization modelling of all well-detected lines was undertaken using Cloudy and is described in Sect. 5. Consideration of the lack of a gradient in the PN O abundances, comparison of the results with the stellar, photometrically-derived metallicity, and relation of the PN to the stellar populations in NGC 5128 are discussed in Sect. 6. Conclusions are collected in Sect. 7. 
Table 1. FORS1 pre-imaging observations.

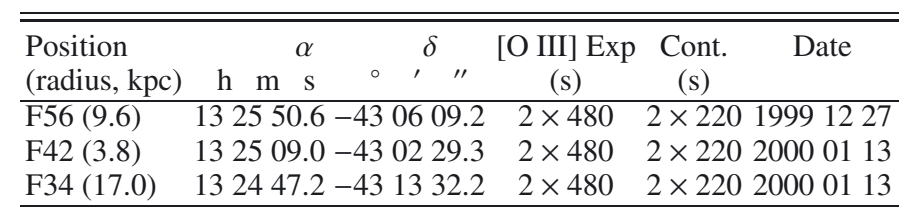

\section{Observations}

\subsection{FORS imaging and spectroscopy}

The European Southern Observatory Very Large Telescope (VLT) FORS1 instrument mounted on VLT Unit Telescope 1 was used for the observations described. FORS1 has a number of modes for imaging; for spectroscopy, long slit, multi-slit, or multi-slit mask are available (see Appenzeller et al. 1998, for details). The multi-object mode (MOS) allows 19 slitlets of height varying from 20 to $22^{\prime \prime}$ to be placed over a $6.8 \times 6.8^{\prime}$ field of view. In the regions of NGC 5128 where PNe were catalogued by Hui et al. (1993a) and (1993b), the surface density is such that there are usually enough PNe to be able to fill the slitlets, thus ensuring an optimal match between target density and instrument multiplex. This match was realised in practice for regions over the bright disk of NGC 5128, whilst in the outer regions, around $50 \%$ of the slitlets could be utilised. Three regions were selected for MOS spectroscopy to fulfill the following criteria: sample the inner and outer regions at a range of effective radii to detect any abundance gradient from the PNe; sample the major and minor axes; ensure that some of the brightest PNe were observed to maximize the probability of detecting the faintest line species; collect as many PN spectra as possible. Not all of these criteria are mutually consistent, but the three regions selected at radial offset distances of 3.4, 8.7 and $15.4^{\prime}$ provided spectra of $51 \mathrm{PNe}$ at a range of $m_{5007 \mathrm{~A}}\left(m_{5007 \mathrm{~A}}=-2.5 \log F_{\lambda}\right.$ $\left(\mathrm{erg} \mathrm{cm} \mathrm{cm}^{-2} \mathrm{~s}^{-1}\right)-13.74$; Jacoby 1989) from 23.5 to $>27.0 \mathrm{mag}$.

\subsubsection{Pre-imaging}

Direct images with FORS1 and a narrow band [O III] filter (called OIII+50, centred at $5005 \AA$ and FWHM $57 \AA$ ) were obtained in December 1999-January $2000^{1}$ in service mode. Table 1 lists details of the imaging observations whose primary purpose was to provide images for the MOS slit assignment using the FIMS tool. The standard resolution collimator was used giving a field of $6.8 \times 6.8^{\prime}$ with a pixel size of $0.2^{\prime \prime}$ per pixel. The field names are taken from those of Hui et al. (1993a). A companion filter (actually a redshifted [O III] filter OIII/6000, centred at $5109 \AA$ with $F W H M 61 \AA$ ) was also used to obtain an [O III] off-band subtraction to confirm the reality of the PNe; all the previously catalogued $\mathrm{PNe}$ in the fields were confirmed as [O III] emission line objects. Seeing at the time of observations was between 0.5 and $1.2^{\prime \prime}$.

\subsubsection{MOS spectroscopy}

FORS1 MOS observations in service mode were conducted in three sessions ${ }^{2}$. Table 2 lists the salient details in chronological order. To cover the wavelength range with useful diagnostic emission lines (essentially from [O II]3726,3729 A to beyond

\footnotetext{
${ }^{1}$ ESO programme 64.N-0219.

2 ESO programmes 64.B-0219, 66.B-0134 and 71.B-0134.
}

[O II]7320,7330 ̊), two grisms were employed: grism 600B (called GRIS_600B+12) has a dispersion of $1.2 \AA / \mathrm{pixel}$ and, for a centred MOS slitlet, a wavelength coverage of 3450 to $5900 \AA$; grism $300 \mathrm{~V}$ (named GRIS_300V+10 and used with a GG435 blocking filter) has a lower dispersion of $2.7 \AA /$ pix and a coverage of 4450 to $8650 \AA$. The overlap region between both spectra is $4500-5900 \AA$ thus allowing at least the strong [O III]4959, $5007 \AA$ lines to be used to tie the two spectra to a common flux scale. The slit width for the campaigns in 2000 and 2001 was $0.8^{\prime \prime}$ whilst the observations in 2003 had a slit width of $1.0^{\prime \prime}$. The resulting spectral resolutions are 4.8 and $6.0 \AA$ for $600 \mathrm{~B}$ and 10.7 and $13.4 \AA$ for $300 \mathrm{~V}$ observations respectively. The supporting observations of the spectrophotometric standard stars for flux calibration, which were taken with a broad slit of $5^{\prime \prime}$ width, are listed in Table 3.

\section{Reduction and analysis}

All the data frames (flats, science frames on PN and standard stars) were bias subtracted using master bias frames provided by the ESO reduction pipeline contemporaneous with the observing data. Standard IRAF ${ }^{3}$ routines were used for the reduction. Pixel-to-pixel flat fields were constructed from dome flats by extracting the 2D area of each slitlet, collapsing in the cross-dispersion direction, box car smoothing in the dispersion direction and dividing the dome flat by the smoothed version. Identical reductions were performed for the bluer (600B grism) and redder (300V grism) spectra. Wavelength calibration was achieved by fitting 4th order Chebyshev polynomials to the dependence of pixel position on wavelength for the arc lamp lines on each slitlet separately. The separate slitlet images, rebinned to an identical wavelength scale, were then recombined into a single image which was corrected for atmospheric extinction. Individual exposures were combined with clipping to remove discrepant pixels caused by detector bad pixels and cosmic ray events. Flux calibration was achieved by observations of one or more spectrophotometric standard stars, the ones applied being listed in Table 3. The standard star spectra were analysed in an identical way to the PN, except that the one slitlet pertaining to the standard star was reduced. In the few cases where a standard star was not available in the same configuration as the PN on the night of observation, a standard star from another night had to be used. In general conditions were good or photometric, and no large flux calibration discrepancies were found between spectra of the same field observed in different runs (see Table 2). Narrow band magnitudes for the spectrophotometric standards were taken from Hamuy et al. (1994) and the PN spectra were flux calibrated using iraf.noao.onedspec routines. Figure 1 shows an example of the reduced multi-slit spectra for field F56. The unequal wavelength coverage of the spectra is dependent on the relative position of the target centred slitlet within the field. Thus a target to the right edge of the field is truncated to the red but extends to lower wavelengths than a target in the centre of the FORS1 MOS field.

Figure 1 demonstrates that there can be more than one PN per slitlet; in some cases this was planned, by placing the slitlet such that two PN from the Hui et al. catalogue lay on the slit, but in some cases in the two inner fields, PN were spectroscopically detected that were not present in the

3 IRAF is distributed by the National Optical Astronomy Observatories, which are operated by the Association of Universities for Research in Astronomy, Inc., under cooperative agreement with the National Science Foundation. 
Table 2. FORS1 PN spectroscopic observations.

\begin{tabular}{|c|c|c|c|c|c|}
\hline Position & $\mathrm{h} \mathrm{m} \mathrm{s} \begin{array}{l}\delta \\
\end{array}$ & Grism & $\begin{array}{l}\text { Exp. } \\
\text { (s) }\end{array}$ & Date & $\begin{array}{c}\text { Seeing } \\
\left({ }^{\prime \prime}\right)\end{array}$ \\
\hline F56 & $132550.6-430609.2$ & $600 \mathrm{~B}$ & $4 \times 2400$ & 20000502 & $0.8-1.2$ \\
\hline F56 & $132550.6-430609.2$ & $300 \mathrm{~V}$ & $2 \times 2400$ & 20000502 & $0.8-1.2$ \\
\hline F42 & 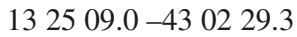 & $600 \mathrm{~B}$ & $2 \times 1500$ & 20010322 & 0.8 \\
\hline F34 & $1324 \quad 47.2-431332.2$ & $600 \mathrm{~B}$ & $2 \times 1500$ & 20010324 & 0.9 \\
\hline F34 & 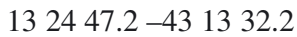 & $600 \mathrm{~B}$ & $2 \times 1500$ & 20010327 & 0.8 \\
\hline F42 & $132509.0-430229.3$ & $600 \mathrm{~B}$ & $2 \times 1500$ & 20010327 & 0.7 \\
\hline F34 & $132447.2-431332.2$ & $300 \mathrm{~V}$ & $2 \times 1320$ & 20030325 & 0.7 \\
\hline F34 & 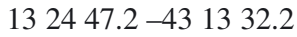 & $600 \mathrm{~B}$ & $8 \times 1320$ & 20030408 & 0.5 \\
\hline F42 & $132509.0-430229.3$ & $300 \mathrm{~V}$ & $2 \times 1320$ & 20030424 & 1.0 \\
\hline F42 & 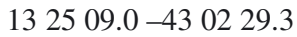 & $600 \mathrm{~B}$ & $3 \times 1320$ & 20030424 & 1.0 \\
\hline F42 & $132509.0-430229.3$ & $600 \mathrm{~B}$ & $3 \times 1320$ & 20030430 & 0.6 \\
\hline F42 & $132509.0-430229.3$ & $300 \mathrm{~V}$ & $2 \times 1320$ & 20030430 & 0.5 \\
\hline F56 & $132550.6-430609.2$ & $300 \mathrm{~V}$ & $2 \times 1320$ & 20030505 & 0.5 \\
\hline F56 & $132550.6-430609.2$ & $600 \mathrm{~B}$ & $4 \times 1320$ & 20030505 & 0.6 \\
\hline F34 & 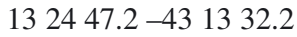 & $300 \mathrm{~V}$ & $2 \times 1320$ & 20030602 & 1.1 \\
\hline F56 & $132550.6-430609.2$ & $600 \mathrm{~B}$ & $2 \times 1320$ & 20030721 & 1.0 \\
\hline F56 & $132550.6-430609.2$ & $300 \mathrm{~V}$ & $2 \times 1340$ & 20030722 & 0.4 \\
\hline
\end{tabular}

Table 3. FORS1 spectrophotometric standard star observations.

\begin{tabular}{lccc}
\hline \hline Star & Grism & $\begin{array}{c}\text { Exp. } \\
\text { (s) }\end{array}$ & Date \\
\hline LTT 7379 & 600B & 25 & 20000502 \\
LTT 7379 & $300 \mathrm{~V}$ & 7 & 20000502 \\
LTT 7379 & $600 \mathrm{~B}$ & 25 & 20010324 \\
LTT 7379 & $600 \mathrm{~B}$ & 25 & 20010327 \\
EG 274 & $600 \mathrm{~B}$ & 30 & 20030408 \\
LTT 7379 & $600 \mathrm{~B}$ & 25 & 20030424 \\
LTT 7379 & $300 \mathrm{~V}$ & 7 & 20030424 \\
LTT 7379 & $600 \mathrm{~B}$ & 25 & 20030430 \\
LTT 7987 & $600 \mathrm{~B}$ & 100 & 20030505 \\
LTT 7987 & $300 \mathrm{~V}$ & 30 & 20030505 \\
LTT 7987 & $300 \mathrm{~V}$ & 30 & 20030602 \\
LTT 7379 & 300V & 7 & 20030722 \\
\hline
\end{tabular}

Hui et al. catalogue, on account of their faintness or proximity to another catalogued PN. These previously uncatalogued PN typically had $m_{5007 \mathrm{~A}}>27 \mathrm{mag}$. On the basis of the strongest line [O III] $5007 \AA$ - the number of PN detectable in the three fields were: 21 in F56; 21 in F42; and 9 in F34. On each slitlet, regions distinct from the PN spectra were located and used to subtract the background, with a 1 st or 2 nd order fit in the cross-dispersion direction. In determining the spectra of the PN, no attempt was made to perform a separate sky background subtraction since there is galaxy background at all slitlet positions, except perhaps for the slitlets at largest galactocentric distance in the outermost field (F34). 1D spectra of each PN were formed by summing the background subtracted signal along the slit; 7-9 pixels $\left(1.4-1.8^{\prime \prime}\right)$ were used to collect around 60 to $70 \%$ of the flux for $0.9^{\prime \prime}$ seeing (assuming a Gaussian PSF). In addition to producing 1D spectra of flux, the same extraction was performed for non-flux calibrated spectra in order to determine the random noise errors on the extracted spectra. No attempt was made to propagate other sources of error, such as flat fielding, into the resultant error vectors.

As is evident from Table 2 all fields were observed on more than one occasion. In order not to lose any information, all the flux calibrated and extracted 1D spectra were employed to form averaged spectra per PN. It was expected that the average should be formed using the inverse of the exposure time

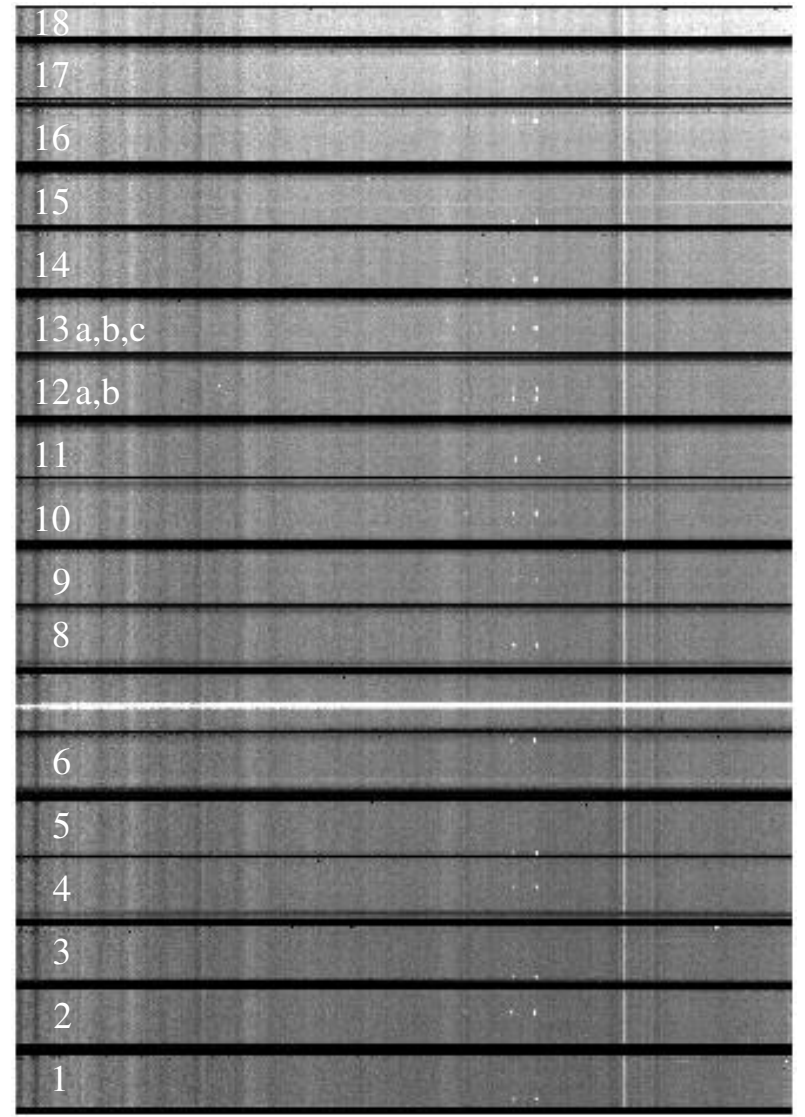

Fig. 1. The reduced MOS spectra for field F56 taken with grism 600B (data from May 2000). Eighteen of the 19 slitlets are shown for a wavelength range from about 3970 to $5520 \AA$ and the numbering of the extracted PN spectra are indicated. Two slitlets show multiple PN spectra. The higher background stellar continuum towards the top of the image corresponds to lower galactocentric distance.

as weight; however the sky transmission, airmass, seeing and moon phase could all affect the resulting noise in a spectrum, so a simple unweighted mean was finally employed. However the F56 600B spectra obtained in 2003 were found to have lower fluxes but similar signal-to-noise ratio $(\mathrm{S} / \mathrm{N})$ as the $2000 \mathrm{ob}-$ servations. These later observations were rescaled in forming 
Table 4. NGC 5128 PN without reliably measured $\mathrm{H} \beta$ fluxes

\begin{tabular}{lcccccc}
\hline \hline MOS Slitlet & $\begin{array}{c}\text { Hui et al. } \\
\text { No. }\end{array}$ & $\begin{array}{c}\alpha \\
(\mathrm{h} \mathrm{m} \mathrm{s})\end{array}$ & $\begin{array}{c}\delta \\
\left({ }^{\prime},{ }^{\prime \prime}\right)\end{array}$ & $\begin{array}{c}\text { Offsets } \\
\Delta \alpha \Delta \delta \\
(\operatorname{arcsec})\end{array}$ & $\begin{array}{c}m_{5007 \mathrm{~A}} \\
\text { mag }^{*}\end{array}$ & $\begin{array}{c}{[\mathrm{O} \mathrm{III]} / \mathrm{H} \beta} \\
\text { approx. }\end{array}$ \\
\hline F56\#7 & 5509 & 132556.41 & -430623.2 & $(+293.6,-314.4)$ & $26.2^{\dagger}$ & 29 \\
F56\#13a & & 132546.50 & -430457.3 & $(+207.3,-228.5)$ & 27.6 & 4 \\
F56\#13c & & 132545.78 & -430453.3 & $(+199.4,-224.5)$ & 27.5 & 13 \\
F42\#12a & & 132515.21 & -430312.5 & $(-135.9,-123.7)$ & 26.5 & 23 \\
F42\#14a & & 132518.20 & -430223.1 & $(-103.1,-74.3)$ & 26.8 & 22 \\
F42\#14c & 132519.24 & -430719.9 & $(-91.7,-71.1)$ & 27.1 & 9 \\
F42\#16b & 132523.13 & -430249.6 & $(-49.0,-100.8)$ & 27.2 & 9 \\
\hline
\end{tabular}

Notes. ${ }^{(*)}$ Spectroscopic magnitude, corrected for slit/pointing losses. ${ }^{(\dagger)}$ Hui et al. (1993b) list $m_{5007 \mathrm{~A}}$ of 25.76 .

the average. For each $\mathrm{PN}$, the various spectra were intercompared before averaging; if a line was found to be significantly discrepant between spectra, a careful examination of the raw 2D spectra was made to determine if a processing step, such as incorrect CR removal in the PN spectrum or the local background, had affected the emission line flux. If a line was deemed to be badly affected, the final spectrum was substituted using only the clean spectrum.

The $300 \mathrm{~V}$ spectra overlap with the $600 \mathrm{~B}$ spectra for the range 4600-5800 $\AA$ allowing the red lines (He I $5876 \AA$, $\mathrm{H} \alpha+[\mathrm{N} \mathrm{II}]$, [S II], etc.) to be placed on the same relative flux scale as the blue lines, primarily using the strong $5007 \AA$ line to scale the spectra. The errors on the final spectra were combined using Gaussian error propagation though these combination steps. The resultant spectra thus have a range of line $\mathrm{S} / \mathrm{N}$ and the magnitude of the propagated errors were checked in two ways: regions of line-free continua were chosen and the rootmean square on the mean was compared with the mean of the statistical errors for the same regions; the rms on the mean value of the fixed [O III] $5007 / 4959 \AA$ ratio was compared with the mean of the errors on this observed ratio from the Gaussian line fits (see Sect. 4.1). It was found that the naively propagated errors over-estimated the real errors on the data values as demonstrated by these two tests; the factor varied slightly between data sets but was around 1.7 for each spectrum. The statistical errors in each spectrum were amended by this amount and are those that are listed as the propagated errors on the measured line fluxes (see Table 11).

In order to examine the stellar spectra at the positions of the $\mathrm{PN}$, an attempt was made to subtract the sky background for the bluer (600B) spectra. The F34 field contains the highest proportion of sky over galaxy background, so was used to remove sky from the exposures of the other fields when F34 and other fields were observed on the same night (observing runs in 2001 and 2003 - see Table 2). The flux calibrated sky spectrum per pixel for the F34 field, excluding the PN spectra, was formed and subtracted from the F56 and F42 data. Some mismatches in terms of the sky lines were noted, particularly [N I], and some of the sky spectra produced poor subtraction in the sense of over-subtraction to short wavelengths. These problematic spectra were not used in forming a mean galactic background spectrum in the two fields F42 and F56.

\section{Results}

\subsection{Individual PN spectra}

The 1D spectra of the 51 PN in NGC 5128 were analysed by interactively fitting Gaussians to the emission lines with a linear interpolation to the underlying galaxy continuum over the line extent. Errors on the line fits were propagated from the flux errors. The extinction correction was determined by comparison with the case B values for $12000 \mathrm{~K}$ and $5000 \mathrm{~cm}^{-3}$ and the Seaton (1979) Galactic reddening law with $R=3.2$ (in the absence of other information on the appropriate reddening law to adopt for NGC 5128), in all cases where at least the $\mathrm{H} \alpha$ and $\mathrm{H} \beta$ lines were detected. The observed line fluxes (normalised to $\mathrm{H} \beta=100$ ) and errors are presented in Table 11 (available electronically). The field number and slitlet numbering of the target is provided, the number from the catalogue of Hui et al. (1993b) together with the $\Delta \alpha, \Delta \delta$ offsets (in arcsec) from the position of the nucleus (taken as the SIMBAD coordinate $\left.13^{\mathrm{h}} 25^{\mathrm{m}} 27.6^{\mathrm{s}}-43^{\circ} 01^{\prime} 08.8^{\prime \prime}(\mathrm{J} 2000)\right)$, the observed logarithmic $\mathrm{H} \beta$ line flux and error, and the $m_{5007 \mathrm{~A}}$ determined from the observed [O III] $5007 \AA$ line flux and error. The extinction correction $c$, and $E_{B-V}$ values are listed in Table 12 (available electronically). The errors on the extinction were not propagated to the dereddened line errors.

Seven PNe with weak emission lines, whose large errors on the $\mathrm{H} \beta$ line precluded reliable determination of line ratios, were also analysed but are not included in Table 11; these are listed separately in Table 4. Except for the PN F56\#7 (5509 in Hui et al. 1993b), the absolute coordinates of these faint PN were determined from the relative positions of the PN on the slitlet with respect to the PN coordinates from Hui et al. (1993b). The $[\mathrm{O} \mathrm{III}] / \mathrm{H} \beta$ ratios are listed in Table 4 where $\mathrm{H} \beta$ was detected; however given the large uncertainty on the $\mathrm{H} \beta$ line flux, these ratios are poorly determined. The PN not detected from the narrow band imaging of Hui et al. (1993b) are all faint $\left(m_{5007 \mathrm{~A}}>26.5\right)$ and are near the centre of the galaxy where the stellar continuum is high. Slit spectroscopy allows lower equivalent width emission lines to be detected and can thus probe deeper than filter imaging.

The comparison between the $m_{5007 \mathrm{~A}}$ magnitudes from this work, converting the measured $5007 \AA$ flux to $m_{5007 A}$, and those from Hui et al. (1993b) is shown in Fig. 2. For Field F56, the observed $m_{5007 \mathrm{~A}}$ magnitudes are on average $0.33 \pm 0.12 \mathrm{mag}$ brighter than the Hui et al. measurements, excluding the two faintest PN. The absolute $\mathrm{H} \beta$ flux calibration was adopted from the May 2000 600B observations; presumably the spectrophotometric standard was taken under slightly poorer conditions, leading to an apparent higher absolute flux for the PN observations. For the F42 field, the $m_{5007 \mathrm{~A}}$ magnitudes are $0.67 \pm 0.21 \mathrm{mag}$ fainter than the Hui et al. ones, which can be attributed to an offset of the slits from the PN positions, since all the F42 observations display lower $m_{5007 \mathrm{~A}}$ than the imaging observations. For the F34 field, the $m_{5007 \mathrm{~A}}$ magnitudes are on average $0.51 \pm 0.21 \mathrm{mag}$ 


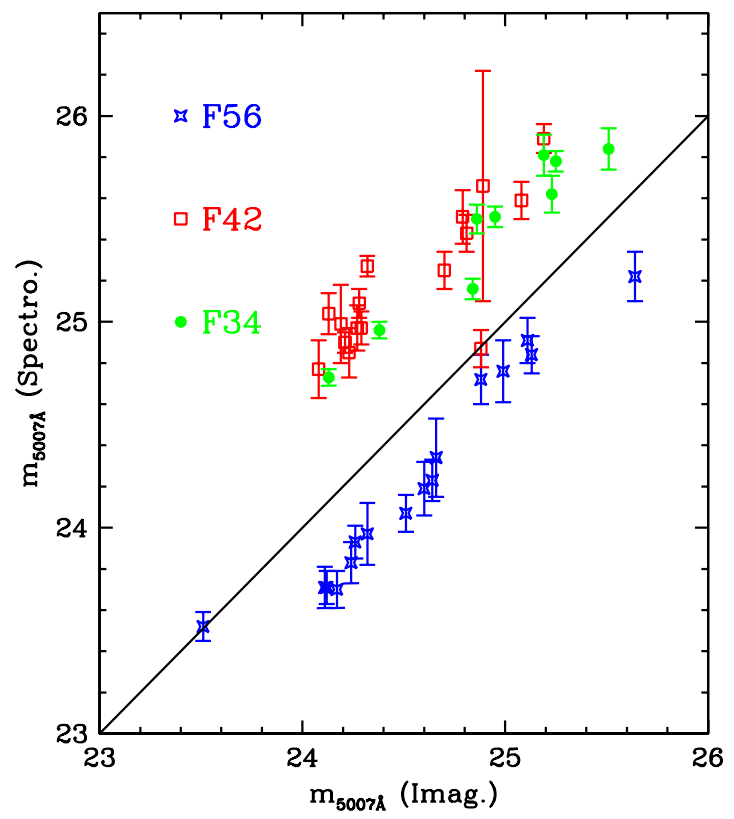

Fig. 2. Comparison of the imaging $m_{5007 \mathrm{~A}}$ magnitude from Hui et al. (1993b) with the equivalent magnitude computed from the observed [O III]5007 ̊ spectroscopic flux converted to magnitude. The PN in the three fields are shown with different symbols, as indicated.

fainter than the imaging magnitudes, which are consistent with $\mathrm{a} \approx 0.2^{\prime \prime}$ slit offset for $0.8^{\prime \prime}$ wide slits in $1.0^{\prime \prime}$ seeing (assuming a Gaussian seeing profile).

\subsection{Region-averaged PN spectra}

Single spectra consisting of the sum of all the observed brighter PN (i.e. those contained in Table 11) in each of the three fields were constructed. The primary motivation was to enable the detection of fainter diagnostic lines (such as He I, [S II], [Ar IV], etc.) which were not detected, or were marginally detected, on the individual spectra. This was also an explorative study to measure how different the derived abundances would be from the summed spectra in comparison with the ensemble of the individual PN abundances. This aspect is of interest for studies of more distant PN populations where all line detections from individual $\mathrm{PN}$ spectra have low $\mathrm{S} / \mathrm{N}$ and summed spectra are mandatory for measurement of abundances. The radial velocity of each PN was measured from the strong lines in the spectrum (principally [O III] 4959 and $5007 \AA$ and H $\alpha$ ) and employed to shift all the spectra in a single region to zero radial velocity. The spectra were then summed and the resulting emission line spectra fitted in the same way as the individual PN spectra (Sect. 4.1). Figure 2 of Walsh et al. (2005) shows the resulting three spectra. Table 5 (available electronically) lists the resulting observed fluxes. The extinction was calculated from the $\mathrm{H}$ line ratios with the same assumptions as for the individual PN spectra (Sect. 4.1) and the dereddened line fluxes are listed in Table 6 (available electronically).

The ionic abundances of the elements corresponding to the detected species can be determined from the dereddened line ratios with respect to $\mathrm{H} \beta$ (Table 6). The electron temperature of the $\mathrm{O}^{++}$emitting region can be directly measured from the $5007 / 4363 \AA$, but also required for abundances is an electron density measure. The only directly determined $N_{\mathrm{e}}$ value is from the $[\mathrm{S}$ II]6716/6731 $\AA$ ratio, which has two disadvantages in that it samples the minority low ionization nebular volume and has a relatively low critical density for collisional de-excitation. Coppeti \& Writzel (1989) show that [S II] $N_{\mathrm{e}}$ measured in PN is similar to $N_{\mathrm{e}}$ measured for higher ionization species, such as [Cl III] and [Ar IV]. Adopting a value of $N_{\mathrm{e}}$ of $5000 \mathrm{~cm}^{-3}$ for the NGC $5128 \mathrm{PN}$ appears to be fair and will not lead to bias on the abundance estimates, unless the density is very high $\left(\gtrsim 20000 \mathrm{~cm}^{-3}\right)$. In the high density case, the [O III] $5007 / 4363 \AA$ line ratio is decreased by collisional de-excitation, leading to too high a value of $T_{\mathrm{e}}$.

Table 7 lists the derived ionic abundances for the summed region spectra. The sources for the atomic data for the collisionally excited lines were taken from the compilation in Liu et al. (2000) and the routines from Storey \& Hummer (1995) were used for the recombination line emissivities of $\mathrm{H}$ and $\mathrm{He}$. The corrections for unseen stages of ionization (ionization correction factors, ICFs) were taken from Kingsburgh \& Barlow (1994). The errors in the total abundances do not take into account the errors in the electron temperature; if these are propagated the errors rise to \pm 0.10 for the $\mathrm{O}$ abundance. The $\mathrm{He} / \mathrm{H}$ abundance is only listed if the lines at 4471 and or $5876 \AA$ were detected; if He II $4686 \AA$ was detected in addition, it was added to derive the total $\mathrm{He} / \mathrm{H}$. If only $\mathrm{He}$ II was detected no $\mathrm{He} / \mathrm{H}$ is listed.

\subsection{Stellar absorption lines}

The slitlets sample a spectroscopic background consisting of sky with a substantial contribution from the galaxy continuum, except for the outermost field, F34. The regions of the slitlets not occupied by planetary nebula spectra can provide the spectrum of the stellar continuum of NGC 5128. The inner fields, F56 and F42, contain no slitlets sampling the sky free of stellar continuum, and so no simultaneous sky subtraction can be performed. For the outer field F34 however, the stellar continuum is very weak and was not detected, as shown by the absence of a gradient in the background with radial offset from the galaxy centre. The 600B F34 spectrum taken on 2003-04-08 was thus used to produce a candidate mean sky spectrum for subtraction from the spectra for the two inner fields (F56 and F42). This sky was interactively subtracted from the F42 and F56 spectra for each slitlet. Good results were found only for the F42 spectra taken in April 2001 and for the F56 field spectra taken in May 2003. These results are attributable to very differing sky contributions to the galaxy spectra at the different observation epochs. For the F56 field, the sky-subtracted data show very low continuum in the five slitlets (1-5) furthest from the galaxy centre; these slitlets were not considered for analysis of the galaxian stellar continuum. Figure 3 shows the resulting stellar continuum from fields F56 (blue line) and F42 (red line), for all the slitlets summed. Strong $\mathrm{Ca}$ II H \& $\mathrm{K}, \mathrm{H} \gamma, \mathrm{H} \beta$ and $\mathrm{Mg}$ I lines typical of an intermediate-old stellar population are clearly visible and are indicated on the figure.

The absorption lines in the individual galaxy continuum spectra for each slitlet were analysed by computing Lick indices (Worthey et al. 1994). The spectra were smoothed with a Gaussian to simulate the $\sim 8 \AA$ resolution of the Lick/IDS stellar spectra and shifted to zero velocity before the equivalent widths were computed. Errors on the indices were computed by propagating the flux errors in the equivalent width determination. A single radial velocity of $580 \mathrm{~km} \mathrm{~s}^{-1}$ was used to shift the spectra to rest wavelength. Errors of $\pm 50 \mathrm{~km} \mathrm{~s}^{-1}$ in this radial velocity cause an offset in the bounds of the Lick indices and can introduce errors up to about $0.2 \AA$ in EW. Three of the 
Table 7. NGC 5128 PN Region spectra - Diagnostics and abundances.

\begin{tabular}{l|rr|rr|rr}
\hline \hline Species & \multicolumn{2}{|c}{ F56 } & \multicolumn{2}{c}{ F42 } & \multicolumn{2}{c}{ F34 } \\
\hline & Value & \pm & Value & \pm & Value & \pm \\
\hline [O III] $(5007+4959) / 4363 \AA$ & 153.1 & 12.6 & 234.0 & 46.2 & 161.8 & 10.0 \\
[O III] $T_{\mathrm{e}}(\mathrm{K})$ & 10970 & 300 & 9620 & 560 & 10780 & 220 \\
$\mathrm{O}^{+}$ & $1.76 \mathrm{E}-5$ & $1.1 \mathrm{E}-6$ & $2.69 \mathrm{E}-5$ & $2.4 \mathrm{E}-6$ & $3.52 \mathrm{e}-5$ & $1.8 \mathrm{E}-6$ \\
$\mathrm{Ne}^{++}$ & $7.93 \mathrm{E}-5$ & $1.6 \mathrm{E}-6$ & $1.03 \mathrm{E}-4$ & $2.5 \mathrm{E}-6$ & $7.28 \mathrm{E}-5$ & $1.9 \mathrm{E}-6$ \\
$\mathrm{He}^{+}$ & 0.173 & 0.019 & 0.142 & 0.023 & 0.109 & 0.070 \\
$\mathrm{He}^{++}(4471 \AA)$ & 0.0094 & 0.0011 & 0.0081 & 0.0016 & 0.0115 & 0.0009 \\
$\mathrm{O}^{++}$ & $3.091 \mathrm{E}-4$ & $4.3 \mathrm{E}-6$ & $4.997 \mathrm{E}-4$ & $8.6 \mathrm{E}-6$ & $3.307 \mathrm{E}-4$ & $5.4 \mathrm{E}-6$ \\
$\mathrm{He}^{+}(5876 \AA)$ & 0.084 & 0.011 & 0.160 & 0.031 & & \\
$\mathrm{~N}^{+}$ & $1.83 \mathrm{E}-5$ & $2.2 \mathrm{E}-7$ & $2.28 \mathrm{E}-5$ & $6.6 \mathrm{E}-7$ & $1.32 \mathrm{E}-5$ & $6.2 \mathrm{E}-7$ \\
$\mathrm{~S}^{+}$ & $4.40 \mathrm{E}-7$ & $4.6 \mathrm{E}-8$ & $1.39 \mathrm{E}-6$ & $1.5 \mathrm{E}-7$ & & \\
$\mathrm{Ar}$ & $1.50 \mathrm{E}-6$ & $6.3 \mathrm{E}-8$ & $1.46 \mathrm{E}-6$ & $2.0 \mathrm{E}-7$ & & \\
\hline $12+\log (\mathrm{O} / \mathrm{H})$ & 8.53 & 0.01 & 8.74 & 0.01 & 8.59 & 0.02 \\
$\mathrm{He} / \mathrm{H}$ & 0.182 & 0.019 & 0.150 & 0.023 & 0.122 & 0.0070 \\
$\mathrm{Ne} / \mathrm{O}$ & 0.257 & 0.006 & 0.206 & 0.006 & 0.220 & 0.007 \\
$\mathrm{~N} / \mathrm{O}$ & 1.04 & 0.07 & 0.85 & 0.08 & 0.38 & 0.03 \\
$12+\log (\mathrm{S} / \mathrm{H})$ & 6.55 & 0.05 & 7.06 & 0.04 & & \\
$12+\log (\mathrm{Ar} / \mathrm{H})$ & 6.49 & 0.04 & 6.44 & 0.06 & & \\
\hline
\end{tabular}

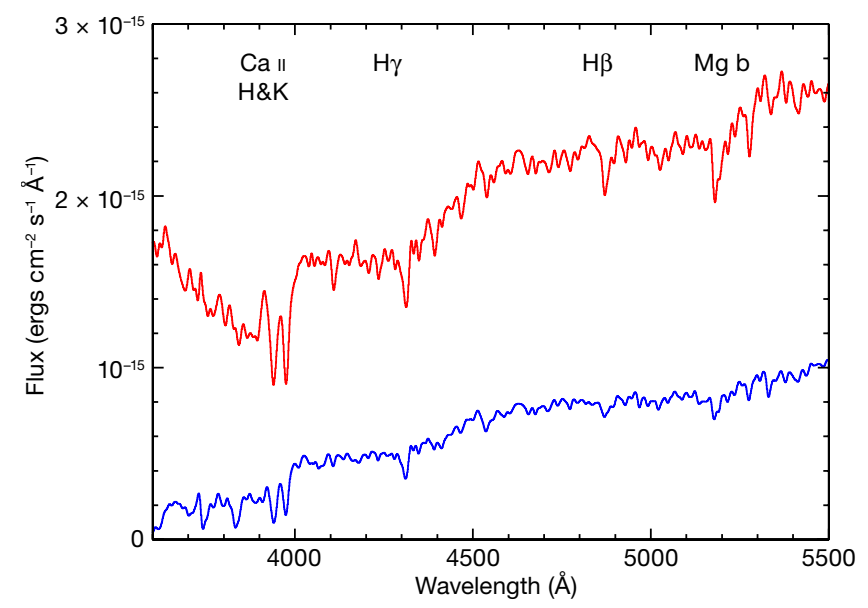

Fig. 3. The observed spectra of the galaxy stellar continuum, summed along the whole slit but excluding the positions occupied by the PN, are shown for fields F42 (upper spectrum, in red) and F56 (lower spectrum, in blue). The strongest absorption lines are identified.

Lick indices $(\mathrm{H} \beta, \mathrm{Mg} \mathrm{b}$ and the combined $\mathrm{Fe}$ index $\langle\mathrm{Fe}\rangle$, defined as $0.5^{*}\left[E W\left(\mathrm{Fe}_{5270 \mathrm{~A}}\right)+E W\left(\mathrm{Fe}_{5335 \mathrm{~A}}\right)\right]$, are plotted in Fig. 4 as a function of the projected position of the slitlet centres from the centre of the galaxy. The $\langle\mathrm{Fe}\rangle$ index shows no radial gradient nor does the $\mathrm{H} \beta$ index, although the latter shows higher scatter to larger projected distance from the nucleus. The $\mathrm{Mg}$ b index shows a weak negative radial gradient, typical of early type galaxies (cf. Davies et al. 1995).

\section{Chemical abundance determination}

\subsection{Extragalactic $P N$ abundances}

The electron temperature $\left(T_{\mathrm{e}}\right)$ is required in order to determine reliable chemical abundances for extra-galactic PNe from the collisionally excited lines on account of the exponential dependence of line emissivity with temperature. The electron density $\left(N_{\mathrm{e}}\right)$ is also required since these lines have collision cross sections dependent on density. In order to avoid large corrections for unseen ionization species a range of lines of different ionization from neutral and singly ionized up to high ionization

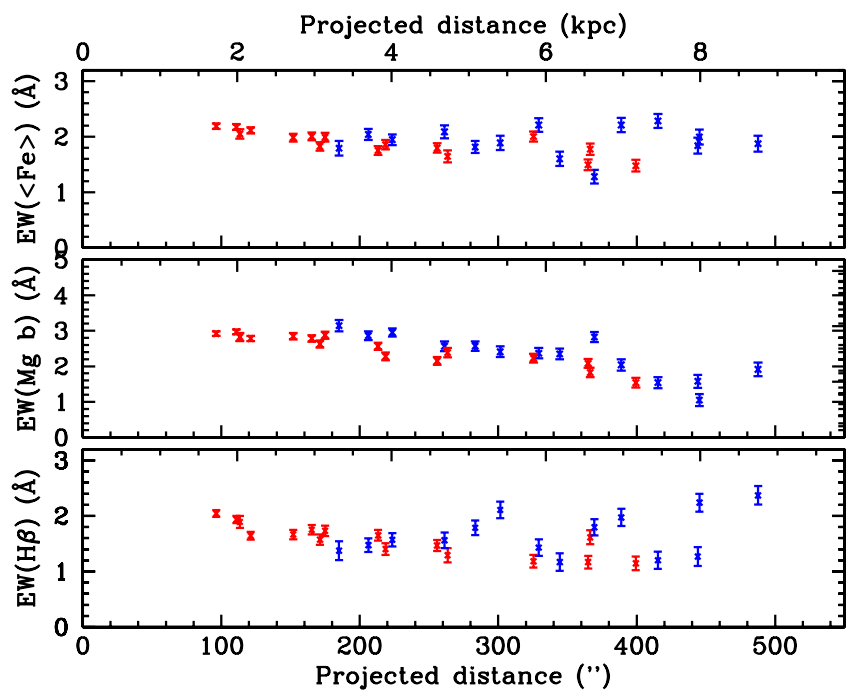

Fig. 4. The Lick indices, $\mathrm{EW}(\mathrm{H} \beta), \mathrm{EW}(\mathrm{Mg}$ b) and $\langle\mathrm{Fe}\rangle$ (defined as $0.5^{*}(E W(5270 \AA)+E W(5335 \AA))$, determined from the stellar continuum in each slitlet, are plotted as a function of the projected distance from the nucleus of NGC 5128, in arcsec (lower axis) and kpc (upper axis). Measurements for field F42 are shown in red and for field F56 in blue.

is also desirable; however the well-established empirical technique using ICFs can compensate for the lack of some ionization species from the optical wavelength range. As is typical for faint and distant PNe such as observed here, these criteria are not fully met. In the absence of measurements of weak $T_{\mathrm{e}}$ and $N_{\mathrm{e}}$ diagnostic line ratios (such as [O III] 5007/4363 $\AA$ and [S II]6716/6731 $\AA$ or [Ar IV]4711/4740 ̊), the practice adopted by Stasińska et al. (1998) was to use the upper limits to the strength of these faint lines to constrain $T_{\mathrm{e}}$ and $N_{\mathrm{e}}$ within reasonable limits, taking typical values for PN from studies in the Milky Way and Magellanic Clouds (e.g. $T_{\mathrm{e}} \sim 12000 \mathrm{~K}, N_{\mathrm{e}} \sim$ $5000 \mathrm{~cm}^{-3}$ ). Jacoby \& Ciardullo (1999) introduced a different approach to abundance determination for extra-galactic PNe (in this case for M 31): that of photoionization modelling using the very well-established Cloudy (Ferland et al. 1998) code. The gains of this latter method are that both strong lines with low 
errors, weak lines with large errors and upper limits can all be used in arriving at a satisfactory model, and the parameters of the central stars (luminosity, $\mathcal{L}$ and effective temperature, $T_{\text {eff }}$ ) are derived as part of the photoionization modelling.

Extra-galactic PNe are spatially unresolved but have known distances and absolute fluxes available though $m_{5007} \AA$ photometry. An input data set for photoionization modelling can be assembled, given some simplifying assumptions in the absence of more detailed information; in particular, the nebular geometry and information on the stellar atmosphere are not available. A black body has been shown to be an acceptable assumption for PN central stars (Howard et al. 1997), which leaves the nebular geometry. Taking a spherical shell of constant density as a baseline, Jacoby \& Ciardullo (1999), hereafter JC99, compared nebular abundances using directly measured $T_{\mathrm{e}}$ and $N_{\mathrm{e}}$ values and ICFs to check that photoionization modelling was indeed a valid approach. The approach was shown to work well. Additional complexity, such as two zone density structure and the presence of dust within the nebula, was found to be required to satisfactorily match the observed spectra in some cases.

Magrini et al. (2004) followed the same procedure as JC99 in determining abundances of PNe in M33. They also tested the method by applying it to the integrated spectra of well-observed Milky Way PNe and compared the model abundances to those from the ICF method, finding agreement within 0.15 dex for $\mathrm{O} / \mathrm{H}$ and 0.3 dex for N/H. In addition Magrini et al. (2004) compared abundances derived from Cloudy models with those from ICFs for three PNe of their M 33 sample. They also considered comparisons employing model atmospheres and an $r^{-2}$ density in the nebular shell, and found no major discrepancies. The technique, involving the development of a large number of models in working to a satisfactory match of observed and model spectrum, is labour-intensive and therefore not suitable for large samples. It also requires simplifying assumptions. The technique, however, is well suited to the present sample of PN spectra in NGC 5128 where the range of abundances is of particular interest and the data set is limited; it also serves as a probe of the star formation history.

Only 10 of the PN spectra presented in Tables 11 and 12 have detectable [O III]4363 $⿱$, enabling direct measurement of the electron temperature, and only 7 have an electron density sensitive ratio ([S II]6716/6731 $\AA$ ) measured. The typical S/N on the weak $4363 \AA$ line $(\leq 3)$ implies that $T_{\mathrm{e}}$ errors of $1250 \mathrm{~K}$ at $11000 \mathrm{~K}$ affect abundance determination, leading to errors of ${ }_{-0.13}^{+0.17}$ on $\log \left(\mathrm{O}^{++} / \mathrm{H}^{+}\right)$. For the PNe without detectable diagnostic line ratios, two approaches are possible: employ the region-averaged spectra to assign $T_{\mathrm{e}}$ and $N_{\mathrm{e}}$ values to the single PN, although these values may be substantially in error for particular nebulae; or, employ photoionization modelling of the strong lines to find the best-matching model that does not violate the upper limit constraints for the weak lines.

\subsection{Modelling procedure}

The simplest possible photoionization model was employed: a black body central star characterized by its temperature and luminosity, the latter given by the $\mathrm{H} \beta$ luminosity derived from the $m_{5007} \AA$ magnitude (Hui et al. 1993b), the reddening and the dereddened $5007 \AA / \mathrm{H} \beta$; a spherical shell with the inner radius set to a small value $(0.005 \mathrm{pc})$ to ensure high ionization emission close to the central star, but not so close that instabilities arise in the model; the outer radius set to a large value $(0.5 \mathrm{pc})$ since the nebulae are expected to be optically thick, given the high luminosity of the PNe observed in NGC 5128 (i.e. within 2 mag of the peak of the PNLF); and an initial set of abundances. For the abundances of $\mathrm{He}, \mathrm{N}, \mathrm{O}, \mathrm{Ne}, \mathrm{S}$ and $\mathrm{Ar}$, the initial values were taken from the ICF analysis for the integrated spectra listed in Table 7 and the $\mathrm{C} / \mathrm{O}$ ratio taken as 0.5 . However these values were immediately allowed to vary to fit the individual spectra (with $\mathrm{C}$ varying in lock-step with $\mathrm{O}$ ) and should not be seen as prejudicing the individual determinations from the higher $\mathrm{S} / \mathrm{N}$ integrated spectra. The initial assumption of an optically thick nebula could also have been relaxed in the modelling process but was not found to be demanded by the fits to the PN spectra. The philosophy was to aim to match the dereddened lines fluxes within the listed errors for all the spectra listed in Table 12 (except F56\#9, F56\#17, F42\#15b and F42\#17 without detected [O II] or He lines), thus $40 \mathrm{PN}$ spectra. These PNe represent the upper 2.1 mag of the PNLF.

The modelling procedure followed closely that outlined by JC99 and also Marigo et al. (2004). An initial default density of $5000 \mathrm{~cm}^{-3}$ was used. The initial estimate of $T_{\text {eff }}$ was taken from He II $4686 \AA / \mathrm{H} \beta$; in the absence of $4686 \AA$, its upper limit and the $5007 \AA / \mathrm{H} \beta$ ratio was used. The presence of $4686 \AA$ enabled $T_{\text {eff }}$ to be refined within a few runs of Cloudy; if $4686 \AA$ was not present, both $T_{\text {eff }}$ and $\mathrm{O} / \mathrm{H}$ had to be adjusted. Then He was adjusted to match the He I lines, with minor adjustments to $T_{\mathrm{e}}$ for the concomitant changes in He II flux. $\mathrm{O} / \mathrm{H}$ and the density were adjusted to match [O II]3727 $\AA$ and, if present, the [O II]7325 $\AA$ quadruplet. For large changes in $\mathrm{O}$ abundance, $\mathrm{Ne}$ and $\mathrm{C}$ were initially changed in proportion. Once the basic physical parameters ( $T_{\text {eff }}$, density, He, O) were roughly determined, $\mathrm{Ne}, \mathrm{N}, \mathrm{Ar}$ and $\mathrm{S}$ were altered to match the observed lines. In the final phase all lines and $T_{\text {eff }}$ and density were subject to small changes to improve the fit, where possible. A guide to the goodness of fit of the observed and predicted line strengths was formed, taking the sum of the line flux differences normalised by the $\mathrm{S} / \mathrm{N}$ : thus larger discrepancies for weak lines could be weighted lower. A discussion of the goodness of the matches and the estimation of errors in presented in Sect. 5.4. In general around 10 iterations per PN was enough to reach a satisfactory match to the spectrum but in more difficult cases (F56\#12b, F42\#10 and F34\#7 for example) up to 30 iterations were required. In total, $\sim 600$ Cloudy models were run to complete this analysis.

\subsection{Results and scrutiny}

Table 8 (available electronically) lists the derived abundances for $\mathrm{He}, \mathrm{N}, \mathrm{O}, \mathrm{Ne}, \mathrm{Ar}$, and $\mathrm{S}$ with respect to $\mathrm{H}$ where lines of these elements were detected, together with the stellar luminosity, black body temperature and the constant shell density. The quality of the derived parameters are broadly classified into three categories based on: He II (and or [O III]4363 A) and [O II] both detected (15 PN: grade a); [O II] but not He II detected (18 PN: grade b); neither He II nor [O II], but at least He I,

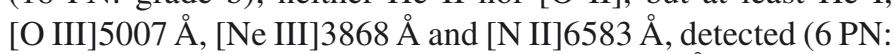
grade c). Ten of the quality "a" spectra had $4363 \AA$ detected and the models tailored to fit the strong lines (i.e. a model was not tweaked to exactly fit the $4363 \AA$ line) were found to match the $4363 \AA$ line within the $1 \sigma$ errors in all cases except one (F42\#16a; 4207 in Hui et al. 1993b). The results presented are not claimed to be unique, as the adopted geometry of the nebulae is the simplest possible and the use of a black body atmosphere does not produce the most satisfactory fits to the level of ionization in detailed photoionization modelling of Galactic PNe (e.g. Wright et al. 2011). However these simple models are 
capable of matching the observed variety of spectra with a very plausible range of abundances, central star temperatures and nebular densities.

Carbon is the most important coolant after $\mathrm{O}$, but no lines of $\mathrm{C}$ were detected so there are no direct constraints on the $\mathrm{C} / \mathrm{H}$ abundance. In the absence of other evidence, the abundance of $\mathrm{C}$ was assumed to be similar to $\mathrm{O}(\mathrm{C} / \mathrm{O}=0.5)$ and to vary in lock-step with $\mathrm{O}$ as the abundance of $\mathrm{O}$ was altered to match the spectrum. Once an adequate model had been found, tests were performed on four of the spectra, altering the $\mathrm{C}$ abundance by a factor 2.5 and refitting for the other species. A maximum difference in $12+\log (\mathrm{O} / \mathrm{H})$ of -0.10 was found for $12+\log (\mathrm{C} / \mathrm{H})$ higher by +0.40 ; for $12+\log (\mathrm{C} / \mathrm{H})$ lower by 0.40 , a maximum difference of +0.02 in $12+\log (\mathrm{O} / \mathrm{H})$ was found. Smaller changes in $\mathrm{N}$ and $\mathrm{Ne}$ abundances were required to match the spectra with these altered $\mathrm{C}$ abundances. Thus the abundances are not very sensitive to modest changes in the assumed $\mathrm{C} / \mathrm{O}$ ratio.

For the best observed PN spectrum (viz. the target with the brightest $m_{5007} \AA$, F56\#2; 5601 in Hui et al. 1993b), a set of Cloudy models were run with NLTE model atmospheres from the Tübingen compilation (Rauch 2003), and also with the presence of dust inside the ionized region. H, He and PG1159 model atmospheres were employed. While the $\mathrm{H}$ model atmosphere showed a much lower $T_{\text {eff }}$, an He or PG1159 atmosphere at $170-180 \mathrm{kK}$ produced satisfactory fits, with the resulting $\mathrm{O} / \mathrm{H}$ lower by up to 0.16 dex. Including dust with an atmosphere model required that $\mathrm{O} / \mathrm{H}$ be reduced by up to 0.05 for a gas-todust mass ratio of 1000; as the primary coolant of the nebula, the lower $\mathrm{O} / \mathrm{H}$ is compensated by the enhanced cooling from dust.

Stasińska (2002) questioned the uniqueness of abundance determinations even in the case that a direct measure of $T_{\mathrm{e}}$ from e.g. [O III] $4363 \AA$ is available. Depending on the temperature of the central star, a strong gradient in the electron temperature can exist within the nebula and a single value of $T_{\mathrm{e}}$ may not be a good representation for the bulk of the emission. The $T_{\mathrm{e}}$ gradient is far less steep for PNe with hot central stars $(\sim 100000 \mathrm{~K})$ than for cooler central stars and the use of a single $T_{\mathrm{e}}$ should yield reliable abundances. Stasińska (2002) presented photoionization models for a Milky Way Bulge PN with a central star temperature of $39000 \mathrm{~K}$ that can be equally well fit by a lower abundance $([\mathrm{O} / \mathrm{H}]=-0.31)$ and by a higher abundance model $([\mathrm{O} / \mathrm{H}]=+0.39)^{4} .95 \%$ of the PNe observed in NGC 5128 appear to have $T_{\text {eff }} \geq 60 \mathrm{kK}$, so the likelihood of a double-valued abundance is very low. However since the lowest $T_{\text {eff }}$ values derived in the Cloudy modelling (Table 7 ) are $\sim 50 \mathrm{kK}$, then uniqueness may be a more significant concern for these few objects.

A concerted attempt was made to fit the spectra of the PNe with the lowest derived $T_{\text {eff }}$ by low and high metallicity $(Z)$ models. Here there is the liberty to change the stellar temperature since the $\mathrm{He}$ II $4686 / \mathrm{H} \beta$ ratio can be taken as an upper limit based on the errors on local lines. Two PN were found that could not be distinguished in terms of lower and higher $Z$ Cloudy models. In the case of F56\#12b (a class b spectrum with the [O II] line detected but not He II), the higher $Z$ solution

\footnotetext{
4 Attempts to reproduce the photoionization fits of Stasińska (2002) using Cloudy 08.01 were not entirely successful. The lower metallicity model could be satisfactorily reproduced with a slightly higher $T_{\text {eff }}$ of $43 \mathrm{kK}$ and lower $\mathrm{O}$ of $[\mathrm{O} / \mathrm{H}]=-1.02$; however the higher metallicity model required $[\mathrm{O} / \mathrm{H}]=+0.86$ and at such high abundance the Cloudy models were very sensitive to extremely small changes in abundances of $\mathrm{O}, \mathrm{Ne}, \mathrm{N}$ and of the nebular size and density. From this numerical experiment, it is tentatively concluded that such extreme conditions are rare.
}

with $12+\log (\mathrm{O} / \mathrm{H})$ of 8.55 for a BB $T_{\text {eff }}$ of $49 \mathrm{kK}$ could not be distinguished from a model with $T_{\text {eff }}$ of $73 \mathrm{kK}$ and $12+\log (\mathrm{O} / \mathrm{H})$ of 8.06 if the density was decreased from $4.0 \times 10^{4}$ to $1.8 \times$ $10^{4} \mathrm{~cm}^{-3}$. For F42\#3 (class c spectrum without [O II] and He II lines detected), a higher $Z$ solution with $12+\log (\mathrm{O} / \mathrm{H})$ of 8.44 for $T_{\text {eff }}$ of $50 \mathrm{kK}$ was indistinguishable from that with $12+\log (\mathrm{O} / \mathrm{H})$ of 8.01 but $T_{\text {eff }}$ of $73 \mathrm{kK}$, for the same density in the shell. For the class c PN, an attempt was made to match each spectrum by a forced lower $Z$ model but no convincing matches could be found; nevertheless, one may exist given the rather weak constraints implied by these lower quality spectra. Conservatively, the higher abundance solutions were adopted (in Table 8) on the grounds that they do not differ strongly from the values for other $\mathrm{PNe}$ at similar radii.

The two PNe with alternate low $\mathrm{O} / \mathrm{H}$ values are not improbable and are comparable to the abundances of PNe in dwarf galaxies (e.g. Dopita \& Meatheringham 1991; Leisy \& Dennefeld 2006); F42\#4 also shows a similarly low value of $\mathrm{O} / \mathrm{H}$ but has a well-detected spectrum. In the context of a giant elliptical galaxy, such low abundance values are surprizing because they imply low metallicity progenitor stars. Radial velocities of these PNe (available for F56\#12b and F42\#4 from Peng et al. 2004) do not show "peculiar" velocities, being within $1 \sigma$ of the mean velocity of NGC 5128. There is no indication of these PNe being obvious "halo" objects or of resulting from a recent lowmass galaxy interloper not yet in dynamical equilibrium with the parent galaxy.

The abundances of the individual PN from Table 8 were combined and compared to the abundances derived from the integrated region spectra with empirically determined temperatures and ICFs presented in Table 7. The means were determined by weighting the individual abundances by $m_{5007 \mathrm{~A}}$. For the combined region F56, the weighted mean abundances from Table 8 for $\mathrm{O}$ agreed to within $0.01 \mathrm{dex}$ and for F34 to within $0.06 \mathrm{dex}$ (weighted means of the $\log$ and the fractional $\mathrm{O} / \mathrm{H}$ abundances were calculated with similar results). For field F42, the discrepancy on the weighted mean of the $\log \mathrm{O}$ abundance was larger, with a value up to 0.22 dex lower than the value in Table 7 . The difference for F42 is quite striking in this comparison; the electron temperature was measured to be $\sim 1000 \mathrm{~K}$ lower than for the other two regions. It is suggested that the value of $T_{\mathrm{e}}$ was underestimated in this merged spectrum due to a poorly determined [O III] 4363 Å flux.

A further check on the integrated region spectra was performed by running photoionization models for the region spectra presented in Table 6 . The abundances were compared to the empirical abundances derived using ICFs (Table 7). Satisfactory Cloudy models could not be fitted without departing from the simplifying assumptions used for the individual PN models. In particular, when the stellar temperature was chosen from the $\mathrm{He} \mathrm{II} / \mathrm{H} \beta$ ratio, this could not be made compatible with the electron temperature derived from the [O III]5007/4363 $\AA$ ratio. If He II4686 $\AA$ matched the observations within the errors, then [O III]4363 $\AA$ was predicted much too strong (higher $T_{\mathrm{e}}$ ); when [O III]4363 $\AA$ matched the model, He II was modelled too low (lower $T_{\text {eff }}$ ). The addition of modest amounts of dust inside the ionized region of the photoionization model to provide extra heating through photoelectric emission from the grains did not resolve this discrepancy. However allowing for these modelling issues, the range of $\mathrm{O} / \mathrm{H}$ was close (within $0.1 \mathrm{dex}$ ) to the empirical values listed in Table 7 for the sum of PNe in fields F56 and F34, but only within 0.2 dex for F42. These demonstrations show that summing $\mathrm{PN}$ spectra provides a useful 
indicator of the mean $\mathrm{O}$ abundance in cases when the line detections have low $\mathrm{S} / \mathrm{N}$, such as for PNe in more distant galaxies. This conclusion is subject to the condition that the PN abundances do not differ strongly, as in this case. Méndez et al. (2005) have presented lower limits on $\mathrm{O}$ and $\mathrm{Ne}$ abundances based on just such a summed spectrum for 14 PNe in NGC 4697 (an elliptical at $11 \mathrm{Mpc}$ ).

\subsection{Towards a comparative study of models}

A number of Cloudy photoionization models were run in order to arrive at an adopted model for each PN matched in Table 8. The models presented cannot be claimed to be unique but represent a feasible match to the spectra in terms of the abundances and physical conditions within the restricted set of parameters (black body atmosphere for the stars, linear density law, etc.). Magrini et al. (2004) and JC99 discussed the accuracy of photoionization models to their PN spectra. In general the "a" quality spectra of the NGC 5128 PN approach those of the fainter sample of the much closer PNe observed in M 33 and M 31 by these authors. Simply adopting the range of models within the statistical errors on the line ratios underestimates the real uncertainties on the abundance determinations. For a selection of models, independent Cloudy runs were performed by two of the authors and the results compared (cf. Jacoby et al. 1997) and with the ICF determinations in the cases where [O III]4363 $\AA$ was detected (using an adopted $N_{\mathrm{e}}$ value of $5000 \mathrm{~cm}^{-3}$ ). While a full error analysis is outside the scope of this work, a limited investigation was attempted. A range of parameter values around the adopted ones were explored to determine the sensitivity of the models, given the errors on the spectra.

It would be very time consuming to perform an investigation of the likely parameter range for all $\mathrm{PN}$ in Table 8, so two $\mathrm{PN}$ were selected. F56\#2 was chosen as a high $\mathrm{S} / \mathrm{N}$ case with both [O III] $4363 \AA$ and He II $4686 \AA$ well detected (a class "a" spectrum in Table 8) and a high stellar temperature. F34\#1 was chosen as a $\mathrm{PN}$ with lower $\mathrm{S} / \mathrm{N}$ and only a marginal detection of He II $4686 \AA$ (class "b" in Table 8) and a lower stellar temperature and representing the typical average quality of spectra. Cloudy models were run for these two exemplars varying a number of parameters about the adopted solution in Table 8: $\mathrm{O}$ abundance varied by \pm 0.2 dex; stellar black body temperature by 10-15\% (20 000 K for F56\#2, with $T_{\mathrm{BB}} 155000 \mathrm{~K}$ and $10000 \mathrm{~K}$ for F34\#1, with $T_{\mathrm{BB}} 71000 \mathrm{~K}$ ); the density by a factor of two; and a model atmosphere rather than a black body. For each modified parameter set, Cloudy models were run to match the spectra within errors as far as possible by freely varying all the other, non-displaced, parameters. Even this limited analysis involved running more than 180 separate Cloudy models.

The results of the comparison of Cloudy models are summarised in Tables 9 and 10 (available electronically). The third columns list the dereddened spectrum from Table 12 and the 4th column the spectrum resulting from the adopted model given in Table 8. The successive columns list the resulting spectra from the incremented parameters. The lower half of each table lists the stellar temperature, density and abundances corresponding to the spectrum in the upper part of the same column; values in Col. 4 being identical to those in Table 8. An overall merit factor, FoM, of the match of the model spectrum with the observed spectrum, taking account of the $\mathrm{S} / \mathrm{N}$ of the fluxes, is defined by:

$\left.F o M=\sum_{i=1}^{n}\left|\left(\left(F_{\text {obs }}-F_{\text {mod }}\right) / F_{\text {obs }}\right)\right| \times\left(F_{\text {obs }} / E r r_{\text {obs }}\right)\right)$ where $F_{\text {obs }}$ and $E r r_{\text {obs }}$ are respectively the dereddened flux and error and $F_{\text {mod }}$ the model flux; the summation is taken over the number of emission lines. Since some lines are in fixed ratio, such as $\mathrm{H}$ lines, a subset of the lines was adopted to dispense with some redundant information (except for the more important lines such as $\mathrm{H} \alpha$ and $\mathrm{H} \beta$, the weak He I lines and the [O III] $4959 \AA$ A line).

Table 9 presents the comparative models for the bright PN F56\#2 (5601 in Hui et al. 1993a). The comparison of the observed and model spectra makes it clear that the He I $5876 \AA$ line is strongly under-estimated, most probably because of the poor removal of the strong $\mathrm{Na}$ I telluric emission. In general satisfactory models could be found, except for the cases of $\mathrm{O}-0.2$ abundance (Col. 6 of Table 9) and $T_{\mathrm{BB}}-20000 \mathrm{~K}$ (Col. 8). What constituted a satisfactory match to the spectrum was not simply given by the FoM value, but certain line ratios, which act as important diagnostics of nebular conditions, e.g. He II/H $\beta$, [O III] $5007 \AA / H \beta$ and [O III] 5007/4363 $\AA$ ), were given higher weight in assessing the quality of the match. It is clear that a range of $\mathrm{O} \pm 0.2$ produces model spectra in which the important [O III] lines are not well-matched, and thus representative errors on $\mathrm{O}$ in the range 0.1 to 0.15 are suggested. Similarly the range of stellar temperature of $\pm 20000 \mathrm{~K}$ and density varying by a factor 2 also over-estimate the allowed range of some of the line ratios. However it is clear from these parameter ranges that the error bars are not necessarily symmetric. H, He and PG1159 atmospheres from Rauch et al. (2003) were input to Cloudy and although the choice of temperatures is not very extensive, a satisfactory fit with the PG1159 atmosphere for $170000 \mathrm{~K}$ was found. However this should not be considered as a best estimate since a comprehensive range of temperature and gravity was not tested.

The comparative models for the fainter PN F34\#1 (2906 in Hui et al. $\left.1993 \mathrm{a}, m_{5007 \mathrm{~A}}=24.96\right)$ are given in Table 10 . Here the number of lines is less than F56\#2, since [O III] $4363 \AA$ and no $\mathrm{He}$ I lines were detected and He II is a limit. The [O II] $7325 \AA$ line is clearly under-estimated in the measured spectrum by comparison with the model spectra. Satisfactory matches could generally be obtained with the ranges of parameters listed. The models with a range of $\mathrm{O}$ of \pm 0.2 are clearly accommodated by the data. Only the lowering of the stellar temperature by $10000 \mathrm{~K}$ (Col. 8 of 10) produced an unsatisfactory fit. A PG1 159 model atmosphere (Rauch 2003) of $70000 \mathrm{~K}$ produced a good fit (Col. 11), but the $70000 \mathrm{~K} \mathrm{He}$ model produced a similar fit with an $\mathrm{O}$ abundance lower by 0.40 dex than the black body.

From this comparison of observed and model spectra with a higher and a lower $\mathrm{S} / \mathrm{N}$, we conclude that the typical dependence of errors on the choice of Cloudy models are generally in the range of $0.15-0.20$ for the $\mathrm{O}$ abundance, around $10-15 \%$ for $\mathrm{He}$ and around 0.2 for $\mathrm{Ne}$ and $\mathrm{N}$. These values are similar to those found by Magrini et al. (2004) comparing abundances from Cloudy models with those of the ICF method. These representative abundance errors are shown in plots, such as Figs. 6 and 7.

\section{Discussion}

\subsection{Individual PN spectra and abundances}

A sample of 51 PNe in NGC 5128 have been observed over a range of projected galactocentric distances from 1.7 to $18.9 \mathrm{kpc}$ and covering the PN luminosity function from the brightest known PN in NGC $5128\left(\mathrm{PN} \mathrm{5601,} m_{5007 \mathrm{~A}}=23.51\right)$ to $4.1 \mathrm{mag}$ 
fainter. The mean $5007 \AA / \mathrm{H} \beta$ ratio is $11.00 \pm 0.55$ (rms on the mean) for the 42 spectra with the highest $\mathrm{S} / \mathrm{N}$. The extrema of the $5007 \AA / \mathrm{H} \beta$ values for this subset are $6.0( \pm 0.67)$ to $18.8( \pm 3.7)$; the value of this ratio is very sensitive to the subtraction of the underlying continuum and the variation of the $\mathrm{H} \beta$ absorption line along the slitlet. The mean logarithmic extinction correction at $\mathrm{H} \beta$ is $0.56 \pm 0.20$. The overall Galactic extinction to NGC 5128 is $E_{B-V}=0.123$ (Burstein \& Heiles 1984) or $E_{B-V}=$ 0.115 (Schlegel et al. 1998), the latter is equivalent to $c=0.17$ for a Galactic extinction law with $R=3.2$ (Seaton 1979). The lowest values of extinction measured in the PNe (Table 12) are compatible with no local extinction in NGC 5128. The histogram of $c$ values peaks at $0.45\left(E_{B-V}=0.30\right)$ and shows no obvious trend with radial offset beyond 200" from the nucleus; the five highest values occur within a radius of $200^{\prime \prime}(3.7 \mathrm{kpc})$. The frequency of higher $c$ values at lower radii is probably due to the high line of sight extinction in the vicinity of the dust lane, rather than any intrinsic PN property (e.g. high intrinsic dust content associated with young PNe, such as NGC 7027; Zhang et al. 2005).

The ratio of $5007 \AA / \mathrm{H} \beta$ does not show any significant overall gradient with projected radial offset from the galaxy centre; the highest values lie in field F42 where the stellar continuum is strongest and removal of the underlying $\mathrm{H} \beta$ is the most critical. There is no evidence for a gradient in $[\mathrm{Ne}$ III $] /[\mathrm{O}$ III $]$ implying a constant $\mathrm{O}^{++} / \mathrm{Ne}^{++}$ratio. If there is any $\mathrm{O}$ enrichment, e.g. during the third dredge-up (Péquignot et al. 2000), or depletion (through the ON cycle, Leisy \& Dennefeld 2006) occurring in the nebular envelopes of the $\mathrm{PNe}$, then this constant value of the ionic ratio implies that any alteration in $\mathrm{O}$ abundance must be accompanied by a corresponding change in Ne. Since $\mathrm{O}-\mathrm{Ne}$ correlation is not predicted by AGB evolution (Karakas \& Lattanzio 2003), the rather constant O/Ne ratio implies that $\mathrm{O}$ enrichment/depletion is not an important effect.

The mean $12+\log (\mathrm{O} / \mathrm{H})$ is $8.52 \pm 0.03$ (median value 8.48 ) and the range of $[\mathrm{O} / \mathrm{H}]$ is -0.66 to +0.31 . The mean $\mathrm{O} / \mathrm{H}$ abundances inside and outside $10 \mathrm{kpc}$ are identical. Of the three PN with the lowest $\mathrm{O} / \mathrm{H}$, two occur close to the nucleus (F42\#4 and $\mathrm{F} 42 \# 10)$ and the other is at $19.5 \mathrm{kpc}(\mathrm{F} 34 \# 2)$ and all have low [O III]/H $\beta$, without notably strong [O II]; F34\#2 has lower N/H. The PN with the highest $\mathrm{O} / \mathrm{H}$ occur in fields 42 and 56 and are distinguished by very high [O III $] 5007 / \mathrm{H} \beta$ but with large errors. The problems in removing the underlying stellar continuum from slit measurements can contribute to large uncertainty on the line fluxes for fainter nebulae in the central regions (see the discussion in Roth 2006), and very high [O III]/H $\beta$ of $>15$ must be viewed with caution since the $\mathrm{H} \beta$ may be considerably underestimated.

The mean $\mathrm{N} / \mathrm{O}$ ratio is $0.51 \pm 0.06$. Four PNe were found with high N/O ratio (F56\#8, F56\#12b, F56\#18 and F42\#4) but only F56\#12b and F56\#18 have $\mathrm{He} / \mathrm{H}$ and N/O which could be classified as characteristic of Type I nebulae (Peimbert 1978). Since F56\#12b was modelled by a lower temperature central star (the lowest of all the PN modelled, Table 8), it may be that optical depth effects and the assumption of a black body may yield misleading results. F56\#18 is perhaps a better candidate for a Type I nebula, having a relatively high stellar temperature and a higher reddening, but this PN does not possess the highest luminosity central star. The brightest PN, and incidentally the nebula with the highest luminosity star (Table 8), has N/O of 0.12 and shows no elevation of this ratio, compared for example to the mean Milky Way value of 0.47 (Kingsburgh \& Barlow 1994). The shorter timescales for PN evolution of Type I PN make them

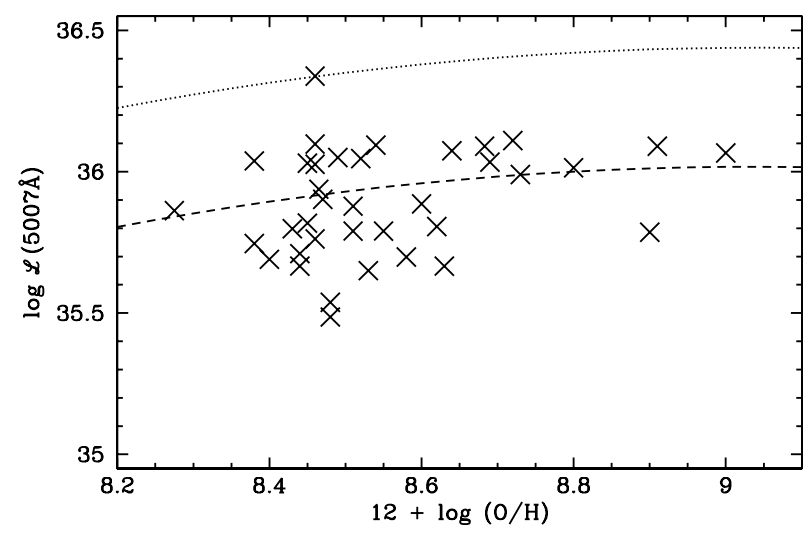

Fig. 5. The dependence of the oxygen abundance of the NGC 5128 PN sample from the Cloudy photoionization modelling (Table 8) on the observed [O III] $5007 \AA$ A luminosity (uncorrected for reddening) from the $5007 \AA$ photometry of Hui et al. (1993b). The dotted line shows the theoretical relation of Dopita et al. (1992) scaled to the brightest PN, while the dashed line shows a second order polynomial fit to all the data points.

minor contributors to a PN population luminosity function, but they contribute about $\sim 10 \%$ of Milky Way PN by number.

The mean oxygen abundances in NGC 5128 PNe are intermediate between values for the LMC $(12+\log (\mathrm{O} / \mathrm{H})$ mean 8.4 , e.g. Leisy \& Dennefeld 2006), and that of the Milky Way (mean 8.68, Kingsburgh \& Barlow 1994). A plot of the dependence of the [O III] $5007 \AA$ luminosity on $12+\log (\mathrm{O} / \mathrm{H})$ (Fig. 5) shows a tendency to increase with the oxygen abundance, in line with the theoretical relation of Dopita et al. (1992) as observationally transformed by Richer (1993; see Fig. 2 and Eq. (1)). A second order fit to the observed points demonstrates a comparable dependence of $[\mathrm{O} \mathrm{III}]$ luminosity on $(\mathrm{O} / \mathrm{H})$ to the theoretical relation and is shown in Fig. 5 by a dashed line.

The region summed spectra provide good detections of a single ionization species of $\mathrm{S}$ and $\mathrm{Ar}$ (Table 6) and lines of these species were detected in a number of the brightest PNe (Tables 11 and 12) allowing useful indications of $\mathrm{S} / \mathrm{H}$ and $\mathrm{Ar} / \mathrm{H}$ (Tables 7 and 8). S and Ar abundances serve an important purpose in that they are not considered to be affected by the AGB nucleosynthesis (e.g. Herwig 2005). Whilst O is the element which is most easily measured in PN since it is the dominant coolant, it can be destroyed by CNO cycling or synthesized during helium burning which impacts its usefulness as a metallicity indicator. Péquignot et al. (2000) have found evidence for third dredge-up $\mathrm{O}$ enhancement in a $\mathrm{PN}$ in the Sagittarius dwarf galaxy $(12+\log (\mathrm{O} / \mathrm{H})=8.36)$, but only at the level of $<0.03$ dex. By comparing the lock-step dependence of Ne and O in PN samples, Richer \& McCall (2008) conclude, in agreement with Karakas \& Lattanzio (2003), that the majority of $\mathrm{PNe}$ do not show any changes in the $\mathrm{O}$ or $\mathrm{Ne}$ abundances across the AGB and $\mathrm{PN}$ transition. The $\mathrm{Ne} / \mathrm{H}$ abundances are well correlated with the $\mathrm{O}$ abundances for the NGC 5128 PNe as shown by Fig. 6 (slope $1.22 \pm 0.09$, or $1.17 \pm 0.04$ excluding the 4 points furthest from the linear relation). This result is similar to the value found by Leisy \& Dennefeld (2006) for the LMC (slope 1.13). The relation between $\mathrm{N} / \mathrm{O}$ and $\mathrm{O} / \mathrm{H}$ is also shown in Fig. 6. There is considerable scatter with a weak but insignificant trend for an anti-correlation, as also found by Magrini et al. (2004) for PNe in M33. The mean $\mathrm{Ar} / \mathrm{H}$ abundance (6.3 for $21 \mathrm{PNe}$ ) is indistinguishable from the mean for 70 PN in the Milky Way (Kingsburgh \& Barlow 1994). 

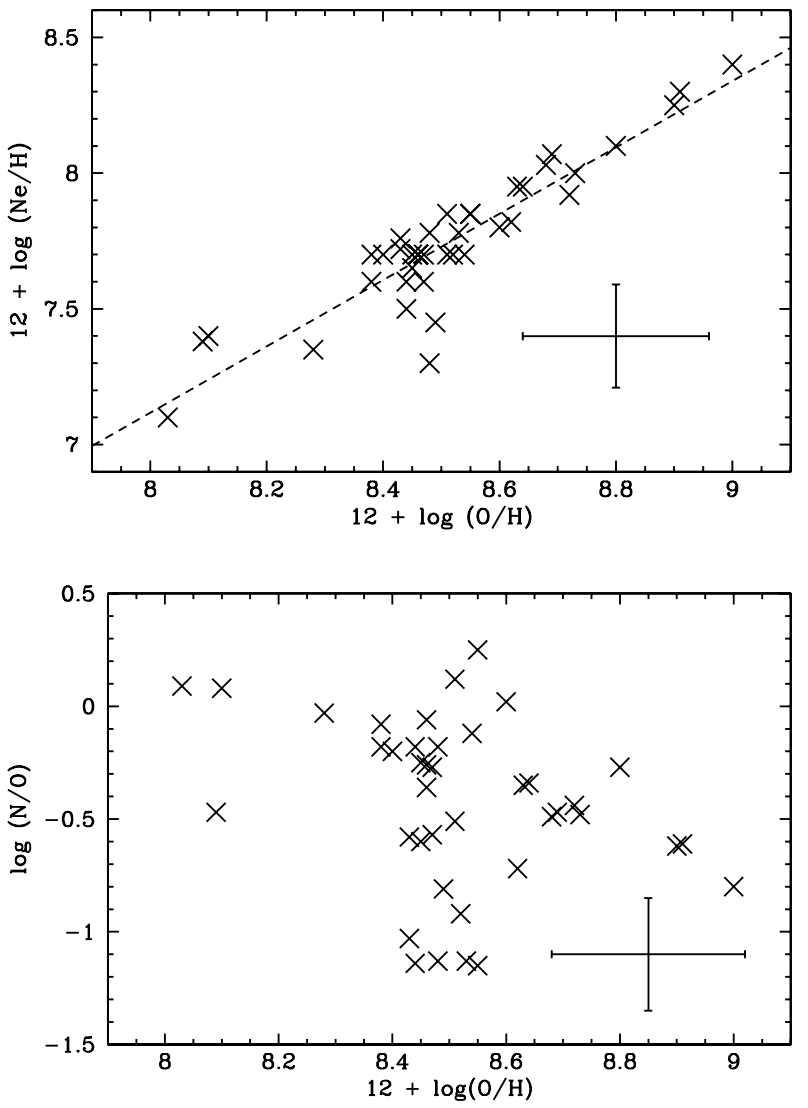

Fig. 6. The relationship between $\log (\mathrm{Ne} / \mathrm{H})$ and $\log (\mathrm{O} / \mathrm{H})$ is shown (upper) and for $\log (\mathrm{N} / \mathrm{O})$ against $\log (\mathrm{O} / \mathrm{H})$ (lower) for the individual NGC 5128 PN abundances tabulated in Table 8. The representative error bars are indicated.

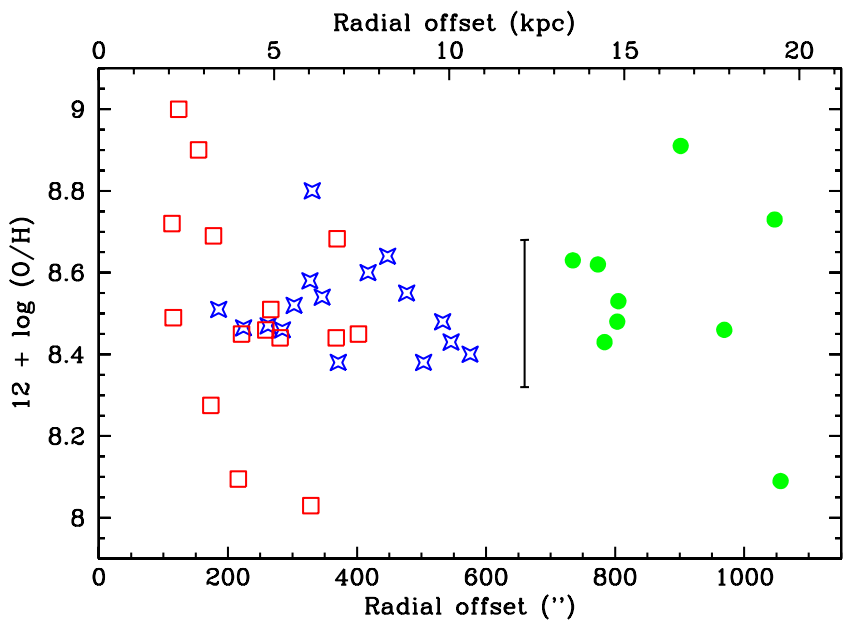

Fig. 7. The radial dependence of the oxygen abundance $(12+\log (\mathrm{O} / \mathrm{H}))$ from the observed PN modelled by Cloudy (Table 8). The points are coded for the three regions as in Fig. 2: F56 by open stars (blue); F42 as open squares (red); F34 by filled circles (green). A representative error bar on the $\mathrm{O}$ abundance is shown.

The mean $\mathrm{S}$ abundance (6.8 for $9 \mathrm{PNe}$ ) also appears to be similar to the mean value from the Kingsburgh \& Barlow (1994) sample. One is led to the surprising conclusion that most of the PN abundances in NGC 5128 are not significantly different from the mean values for Milky Way PNe.

\subsection{PN 5601}

PN 5601 (F56\#2) is the brightest known PN and the best-studied individual PN in NGC 5128, and indeed of all PN known beyond the Local Group. Walsh et al. (1999) measured long slit observations of this PN amongst a few others and the major difference between the spectrum presented in Table 11 is the $\mathrm{H} \alpha / \mathrm{H} \beta$ ratio and the higher $\mathrm{He} \mathrm{II} / \mathrm{H} \beta$ ratio. The earlier observations were taken with a fixed slit position angle over a considerable range of parallactic angle and suffer from wavelength dependent flux loss from the slit. This is demonstrated by the very low value of extinction derived. The value of extinction derived for this nebula is now seen to be quite large, but only slightly higher than the mean value for all the observed PN $\left(E_{B-V}=0.32\right)$, giving no evidence that it is intrinsically dustier than the fainter PNe. There is some expectation from examples of Milky Way PNe, that young, high mass, optically thick PN (cf. NGC 7027, Zhang et al. 2005; NGC 6302 Wright et al. 2011) have higher intrinsic dust extinction.

\subsection{Abundance gradient from $P N e$}

One of the primary aims of this study was to investigate any abundance gradient of the $\alpha$-elements, as revealed by the PN spectra, and to compare it to gradients in the stellar properties such as the metallicity distribution function. A plot of the variation of $\mathrm{O} / \mathrm{H}$ vs. projected radial offset (Fig. 7), for the PNe with $\mathrm{O} / \mathrm{H}$ values from the Cloudy models (Table 8 ), shows two particular features. First, the range of $\mathrm{O} / \mathrm{H}$ values is similar in the inner regions (projected radius, $\mathcal{R}<4 \mathrm{kpc}$ ) to the range in the outer regions $(>13 \mathrm{kpc})$. Second, there is no evidence of a gradient in $\mathrm{O} / \mathrm{H}$. A least squares fit confirms this; the linear correlation coefficient is -0.07 for 40 points. Rather, there is a common mean value of 8.52 for the inner and outer points (considered as less than and greater than $\mathcal{R}$ of $10 \mathrm{kpc}$ ). However, the highest $\mathrm{O} / \mathrm{H}$ values do occur at $\mathcal{R}<4 \mathrm{kpc}$, in the inner field F42. Although our sample is not large, the result that there is no significant $\mathrm{O} / \mathrm{H}$ gradient in NGC 5128 appears to be robust.

The distribution of $[\alpha / \mathrm{Fe}]$ vs. [Fe] has an important role in determining the enrichment history of a galaxy (e.g. Matteucci $\&$ Recchi 2001). O, as representing the $\alpha$ elements, is primarily a core collapse $\mathrm{SN}$ product released early in star formation activity, whilst Fe, as the chief product of Type Ia SNe, is released over many Gyr. Figure 8 shows the Fe vs. Mg b EW plot for the integrated spectra of regions F42 and F56 with several tracks from Thomas et al. (2003) models for selected metallicity and $[\alpha / \mathrm{Fe}]$ ratio. To be compatible with Harris et al. (2000), who calibrated their metallicities by assuming that their stars were as old as those in old Galactic globular clusters, then the models must be compared at an age of 15 Gyr. In this case the datapoints for fields F42 and F56 lie close to the tracks of $[\alpha / \mathrm{Fe}]=$ 0.3 , giving an $[\alpha / \mathrm{Fe}]$ ratio of 0.25 , and show metallicities [Z] around -0.6 (there are no tracks at metallicity intermediate between -0.33 and -1.35 in Thomas et al. 2003). If, however, the age of the stars were younger (as indicated e.g. by Rejkuba et al. 2005,2011 ), the derived metallicities would be about 0.2 higher, giving an average value of -0.4 . This would imply an oxygen abundance relative to solar of -0.15 , a value that is consistent with the mean $\mathrm{O}$ abundance of -0.17 with respect to the solar value (Scott et al. 2009).

These abundances can also be compared with the MDF from Harris \& Harris (2002) in three fields at $7.6 \mathrm{kpc} \mathrm{SW}$ of the centre, at $20 \mathrm{kpc} \mathrm{S}$ and $29 \mathrm{kpc} \mathrm{S}$ (distances rescaled to $3.8 \mathrm{Mpc}$ ). Figure 9 shows the histogram of all the $\mathrm{PN} \mathrm{O} / \mathrm{H}$ abundances 


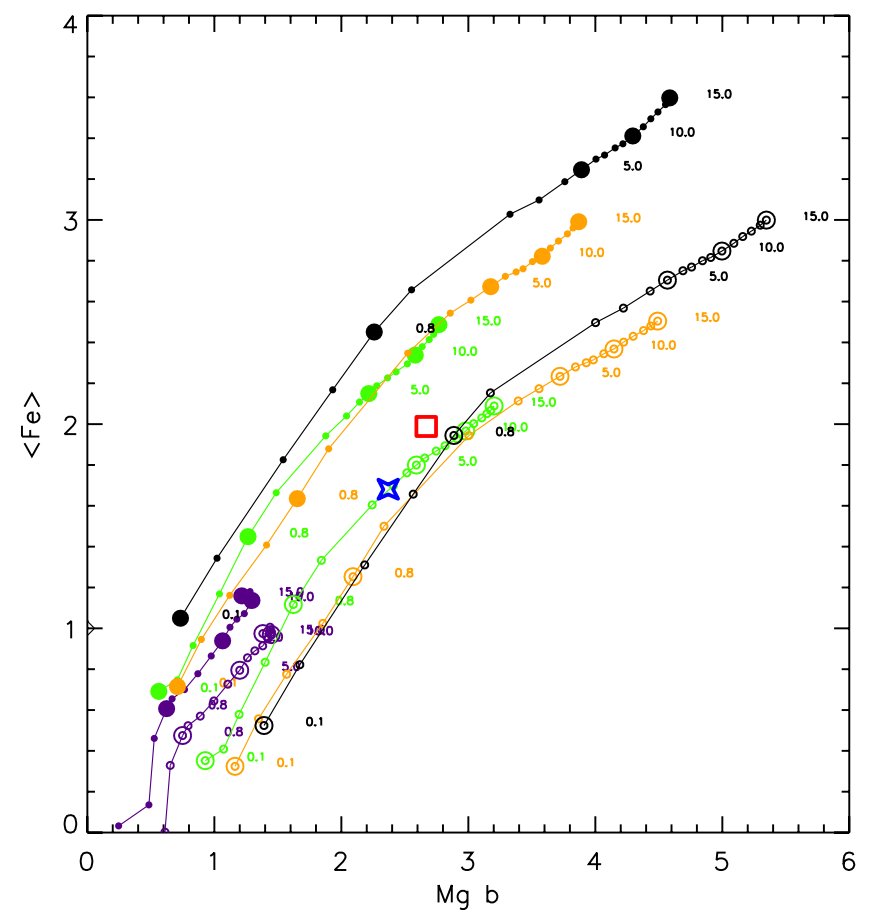

Fig. 8. The Lick index $\langle\mathrm{Fe}\rangle$ is plotted against $\mathrm{Mg} \mathrm{b}$ for the integrated spectra of the two inner fields F42 (box, in red) and F56 (open star, in blue). Two families of tracks from Thomas et al. (2003) for $[\alpha / \mathrm{Fe}]$ of 0.3 (open symbols) and 0.0 (filled symbols) are shown for metallicity [Z] of $-1.35,-0.33,0.0$ and 0.35 by lines with points. The lower set of four lines have $[\alpha / \mathrm{Fe}]=0.3$, and the upper ones $[\alpha / \mathrm{Fe}]=0$. Models with $[Z]=0.35$ are indicated in black, $[Z]=0.0$ in orange, $[Z]=-0.33$ in green and $[Z]=-1.35$ in magenta. The ages in Gyr along each track are indicated.

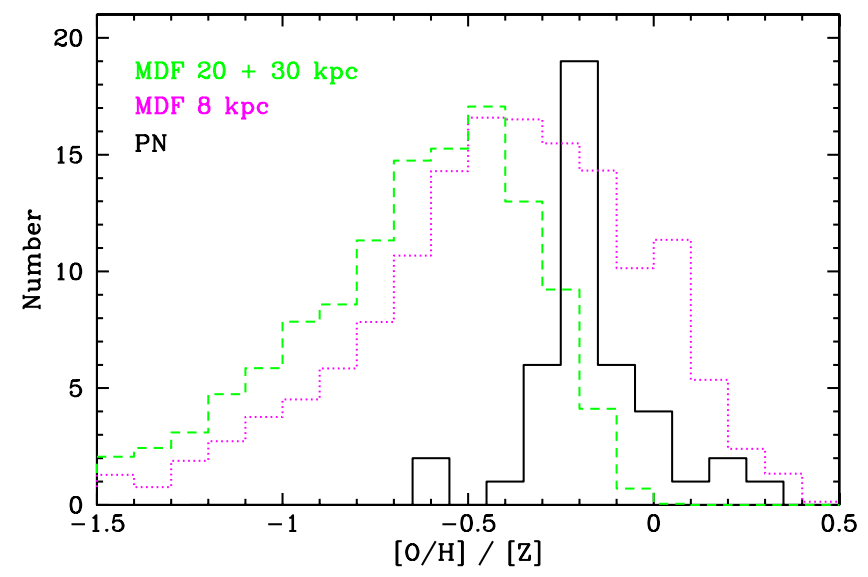

Fig. 9. The histogram of the $\log \mathrm{O} / \mathrm{H}$ abundance relative to Solar is plotted for all PN with $\mathrm{O} / \mathrm{H}$ determined from Cloudy models (Table 8) as a continuous line (black). For comparison the stellar metallicity distribution function (MDF) from Harris \& Harris (2002) is plotted in two regions: an inner region $(8 \mathrm{kpc})$ dotted (magenta); a composite of two outer regions at 20 and $30 \mathrm{kpc}$ dashed (green).

with respect to Solar overplotted against the completeness corrected MDFs in the inner field $(7.6 \mathrm{kpc})$ and two combined outer fields (Table 1 of Harris \& Harris 2002). The PN sample covers the range around the inner and outer fields of Harris et al. (2002). A shift of -0.25 is required to align the peak of the $\mathrm{PN} \mathrm{O} / \mathrm{H}$ distribution with the stellar MDF in the inner field (magenta histogram). However it is strongly apparent that the distribution of $(\mathrm{O} / \mathrm{H})$ for the $\mathrm{PNe}$ is much narrower than for the stellar metallicity, so matching the distribution peaks may not be a justifiable simplification. Splitting the PN into two samples with positions greater and less than $10 \mathrm{kpc}$ shows a shift in the peak value of $(\mathrm{O} / \mathrm{H})$ of $\sim-0.2$ for the inner field and $\sim-0.3$ for the outer field, although the number of PNe with abundances is lower in the outer field (11). For the full PN sample, with an average $\mathrm{O}$ abundance of -0.17 compared to Solar, the total metallicity is then -0.4 assuming a mean $[\alpha / \mathrm{Fe}]=0.25$. Harris et al. (2002) find an average metallicity of -0.6 in their outer fields, which becomes -0.4 assuming an age of $8 \mathrm{Gyr}$; thus taken at face value, assuming the metallicity of the field stars is that of the PN, the age of these stars in NGC 5128 is around 10 Gyr.

Both methods - matching observed spectral indices to stellar population models and comparison with photometrically derived stellar metallicity distributions - provide an average $[\mathrm{O} / \mathrm{Fe}]$ ratio of 0.25 at a stellar metallicity (viz. $[\mathrm{Fe} / \mathrm{H}]$ ) of around -0.4 , and an average age of 8 Gyr. This single value is obviously a simplification for the behaviour in a whole galaxy, but demonstrates that the $[\mathrm{O} / \mathrm{Fe}]$ vs. $[\mathrm{Fe} / \mathrm{H}]$ value for $\mathrm{NGC} 5128$ is within the range of $\alpha$-enhanced stellar metallicities for early type galaxies (cf. Thomas et al. 2003). The modelling of the colour-magnitude diagram by Rejkuba et al. (2011) also independently favours $\alpha$-enhanced stellar abundances in NGC 5128.

However it can be argued that while all the stars are represented in the integrated stellar spectra and photometric surveys, only a subset of these stars become PNe. So, the mean values of the two samples of the $\mathrm{PN}[\mathrm{O} / \mathrm{H}]$ and the stellar $[\mathrm{Fe} / \mathrm{H}]$ in Fig. 9 are not necessarily strictly comparable. In particular the PNe may derive from a younger population, on average. Only the higher $Z$ stars produce observable PN, while the stellar continuum is comprised of stars of all metallicities. Overall the occurrence of PN is highly delimited relative to all the stars in a galaxy and in addition the PN central star masses also have a very restricted range relative to all white dwarfs. Thus the $\mathrm{PNe}$ may only come from a young population; that would explain the tendency toward both higher $Z$ (Fig. 9) and higher central star masses (see Fig. 10). The old stars, which have lower $Z$ on average, fail to produce PNe because their masses are too low.

Chiappini et al. (2009) present a careful comparison of the spectroscopically determined abundances of Milky Way Bulge stars with Bulge planetary nebulae. Their sample of $166 \mathrm{PN}$ in the Bulge with well-determined abundances shows a broad distribution of $12+\log (\mathrm{O} / \mathrm{H})$ from about 8.2 to 8.9 , with a mean of 8.57 , rather similar to the mean value and range determined here in NGC 5128. However the $\mathrm{O} / \mathrm{H}$ abundance collated from various spectroscopic studies of Bulge giant stars shows a lower value by $\sim 0.3$ dex. Various suggestions are offered by Chiappini et al. (2009) for this discrepancy, but none appears to be conclusive. Comparing $\mathrm{O}$ from the Bulge $\mathrm{PN}$ with $[\mathrm{O} / \mathrm{Fe}]$ from the stars, implies $\left[\mathrm{O}_{\mathrm{PN}} / \mathrm{Fe}_{\text {Stars }}\right] \sim-0.1$. This value is not strictly comparable to that in NGC 5128 since $[\mathrm{Fe} / \mathrm{H}]$ is derived from photometry; spectroscopy of individual stars in NGC 5128 is ruled out until larger telescopes are available. Taken at face value the difference in $[\mathrm{O} / \mathrm{Fe}]$ could imply that the formation history of NGC 5128 as a classical giant elliptical, is dissimilar to that of the Bulge; however the discrepancy in $\mathrm{O}$ abundances of Bulge PN and giants found by Chiappini et al. (2009) weakens this conclusion as does the large spread in stellar Fe abundances (in both NGC 5128 and the Bulge).

\section{4. $P N$ and the star formation history}

One of the advantages of running Cloudy models to determine the PN abundances is that the central star luminosities and 


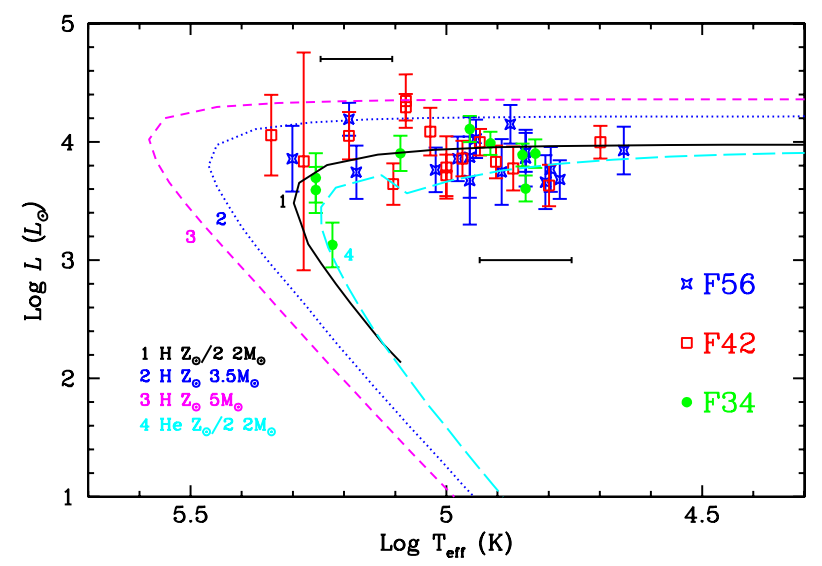

Fig. 10. An HR diagram showing the black body $\log L$ vs. $\log T_{\text {eff }}$ coordinates of the central stars of the PNe with spectra modelled by Cloudy (Table 8) and a selection of low mass $\mathrm{H}$ and He burning model tracks from Vassiliadis \& Wood (1997). The points are coded (and coloured) for the three regions (F42, F56 and F34) as in Fig. 2. Representative error bars on temperature at higher and lower temperatures are shown; the error bars in $\log L$ were computed from the errors on dereddened absolute $\mathrm{H} \beta$ flux in Table 12.

temperatures are derived. Since the PNe are optically thick, the match to the spectrum effectively provides the temperature and the $m_{5007} \AA$ photometry, combined with the reddening, provides the luminosity. Figure 10 shows the $\log L, \log T_{\text {eff }}$ points for the 40 PNe modelled (Table 8). The $\log L$ values span a range of a factor 6, but are, not surprizingly, at the high end for PNe central stars, since only the top few magnitudes of the PNLF is explored by these data. No radial trend in the central star luminosities is apparent, paralleling the lack of an $\mathrm{O}$ abundance gradient (Fig. 7). A few evolutionary tracks from Vassiliadis \& Wood (1997) for higher mass progenitor stars are shown on Fig. 10, although there is currently no information on whether the central stars are on the $\mathrm{H}$ or He burning track. For the $\mathrm{H}$ burning tracks the bulk of the points match stars with masses above $2 M_{\odot}$.

There are multiple routes in terms of stellar mass, metallicity and mode of turn-off from the AGB (H or He burning track) to reach the same PN luminosity, so relating a given $\mathrm{PN}$ in a galaxy to its progenitor star formation episode is non-trivial. Marigo et al. (2004) have modelled effects of stellar populations on the [O III] cut-off of a population of PN, as measured for the PNLF. They find that the peak [O III] luminosity is emitted by stars with initial (main sequence) masses of about $2.5 M_{\odot}$ with a very strong dependence with age. PN from higher mass progenitors, although being intrinsically luminous, evolve very rapidly and so make a minor contribution to the bright end of the PN population. In addition, Ciardullo \& Jacoby (1999) showed that the intrinsically massive and luminous progenitors are prolific dust producers, and therefore self-extinct. Thus the most massive progenitors will generally appear as rare and faint $\mathrm{PNe}$ in a galactic sample. Ciardullo et al. (2002) suggest that the progenitors of the most luminous PNe in galaxies, if they are not formed from stars younger than $\sim 1$ Gyr, may occur predominantly in binary systems.

In principle the calibration of PN luminosity vs. stellar progenitor mass can be used to identify the age of the starburst giving rise to an observed PN population. If indeed the bulk of the PN observed in this study arise in progenitors of about $2.5 M_{\odot}$, then from the grid of evolutionary tracks of low mass stars of Girardi et al. (2000) one can infer a progenitor age of $0.7 \mathrm{Gyr}$ (see Fig. 11 derived from the Girardi et al. tracks for $\left[Z / Z_{\odot}\right] 0.0$,

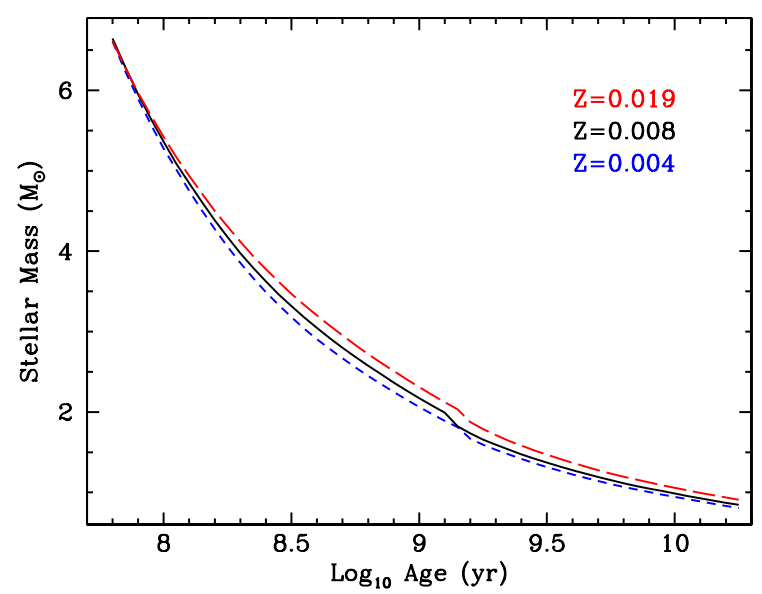

Fig. 11. The age at which a star terminates the AGB is shown as a function of the initial main sequence stellar mass for three metallicities of 0.019 (long-dashed line, red), 0.008 (Solar metallicity; continuous line, black) and 0.004 (approximately LMC metallicity; short-dashed line, blue). The curves are derived from the stellar evolution tracks of Girardi et al. (2000).

-0.4 and -0.7 and the stellar age to AGB termination). Such an age would place the $\mathrm{PN}$ in a very recent star formation episode. This seems rather unlikely since there is little evidence for an extensive stellar population so young. A young (2-4 Gyr) minority $(20-30 \%)$ component was inferred from the HST stellar photometric studies of Rejkuba et al. (2005 and 2011), but not with stars younger than 2 Gyr as appears to be required to match the PN. Younger stars are brighter and so should have been well-detected in optical photometry, ruling out their presence in NGC 5128.

If the PNe studied here belong to this minority younger population of age 2-4 Gyr, then the deduced stellar masses, as indicated by the HR diagram of the PN central stars in Fig. 10, are still unexpectedly high. However since these PN belong to the bright end of the luminosity function, there is a selection effect in favour of the more luminous (viz. highest mass) PN progenitor stars. If however the observed $\mathrm{PNe}$ arose from an intermediate age population (5-8 Gyr), the progenitor stars would only have a mass of $\sim 1.2 M_{\odot}$ and the discrepancy between their deduced masses and age is more difficult to reconcile with evolutionary tracks of single low mass stars (e.g. Vassiliadis \& Wood 1997). It would be profitable to study spectroscopically a group of lower luminosity PNe in NGC 5128 to determine if their properties differ from the high $L$ PNe presented in this study.

\section{Conclusions}

Low resolution spectroscopy of $51 \mathrm{PN}$ in NGC 5128 at a range of galactocentric distance of 2-20 kpc have been obtained with the ESO VLT and FORS1 instrument in multi-slit mode (MOS) in three fields. The PN are drawn from the upper 4 mag of the $\mathrm{PN}$ [O III] luminosity function. The emission line spectra have been analysed and lines typical of ionization by hot PN central stars have been measured. The weak [O III] $4363 \AA$ line was just detected in $20 \%$ of the PNe. In order to determine element abundances for a larger fraction of the observed $\mathrm{PNe}$, photoionization modelling was conducted with Cloudy for 40 PN with the highest quality spectra (representing the upper 2 mag of the PNLF). $\mathrm{He}, \mathrm{N}, \mathrm{O}$ and $\mathrm{Ne}$ abundances were determined for all the PNe, and $\mathrm{S}$ and Ar for about half of the sample. For the most reliably estimated element, oxygen, no radial gradient is seen and 
the slope of $\mathrm{O} / \mathrm{H}$ vs. projected radius is identical inside and outside of $10 \mathrm{kpc}$. The range of $[\mathrm{O} / \mathrm{H}]$ in the sample spanned -0.66 to +0.31 dex with the mean $12+\log \mathrm{O} / \mathrm{H}$ of 8.52 (median 8.48 ).

The PN O abundances were compared to the stellar abundance measured from Lick indices from the continuum spectroscopy (i.e. along the MOS slitlets not occupied by PN emission) and from resolved star photometry from the literature. If the stars in NGC 5128 have an average age of 8 Gyr, the stellar and PN metallicities agree in the outer parts of NGC 5128 with average values around -0.4 Solar. The deduced masses of the PN central stars imply progenitor masses above $2 M_{\odot}$, favouring their formation from a very young component of the intermediate age stellar population with age $\$ 5$ Gyr. This discrepancy between the age of the stellar population and the mass of the PN progenitor stars is in line with other studies of the high mass end of the PN luminosity function.

Acknowledgements. We would like to thank the staff of Paranal for the very efficient conduct of the service observations in programmes 64.N-0219, 66.B-0134, 67.B-0111 and 71.B-0134. We thank the anonymous referee for many helpful suggestions, including the one that led to development of the comparative study of models.

\section{References}

Appenzeller, I., Fricke, K., Fürtig, W., et al. 1998, The Messenger, 94, 1 Arnaboldi, M., Freeman, K. C., Okamura, S., et al. 2003, AJ, 125, 514 Baade, W., \& Minkowski, R. 1954, ApJ, 119, 215

Beasley, M. A., Bridges, T., \& Peng, E. W. 2008, MNRAS, 386, 1443 Burstein, D., \& Heiles, C. 1984, ApJS, 54, 33

Chiappini, C., Górny, S. K., Stasińska, G., et al. 2009, A\&A, 591, 610

Ciardullo, R., \& Jacoby, G. 1999, ApJ, 515, 191

Ciardullo, R., Jacoby, G. H., Ford, H. C., et al. 1989, ApJ, 339, 53

Ciardullo, R., Feldmeier, J. J., Jacoby, G. H., et al. 2002, ApJ, 577, 31

Ciardullo, R., Sigurdsson, S., Feldmeier, J. J., et al. 2005, ApJ, 629, 499

Coppeti, M. V. F., \& Writzel, B. C. 2002, A\&A, 382, 282

Davies, R. L., Sadler, E. M., \& Peletier, R. F. 1993, MNRAS, 262, 650

Dopita, M. A., \& Meatheringham, S. J. 1991, ApJ, 377, 480

Dopita, M. A., Jacoby, G. H., \& Vassiliadis, E. 1992, ApJ, 389, 27

Dufour, R., van den Bergh, S., Harvel, C. A., et al. 1979 AJ, 88, 296

Ferland, G. J., Korista, K. T., Verner, D. A., et al. 1998, PASP, 404, 166

Ferrarese, L., Mould, J. R., Stetson, P. B., et al. 2007, ApJ, 654, 186

Girardi, L., Bressan, A., Bertelli, G., et al. 2000, A\&AS, 141, 371

Graham, J. A. 1979, ApJ, 232, 60

Hamuy, M., Walker, A. R., Suntzeff, N. B., et al. 1994, PASP, 106, 566

Harris, G. L. H. 2010, PASA, 27, 475

Harris, W., \& Harris, G. L. H. 2002, AJ, 123, 3018

Harris, H. C., Harris, G. L. H., \& Hesser, J. E. 1988, in IAU Symp. 126, eds. J. E.

Grindlay, \& A. G. Davis Philip (Dordrecht: Kluwer), 205

Harris, G. L. H., Harris, W. E., \& Poole, G. B. 1999, AJ, 117, 855

Harris, G. L. H., \& Harris, W. E. 2000, AJ, 120, 2423

Harris, G. L. H., Harris, W. E., \& Geisler, D. 2004, AJ, 128, 723

Harris, W. E., Harris, G. L. H., Barmby, P., et al. 2006, AJ, 132, 2187

Harris, G. L. H., Rejkluba, M., \& Harris, W. E. 2010, PASA, 27, 457

Herwig, F. 2005, ARA\&A, 43, 435

Howard, J. W., Henry, R. B. C., \& McCartney, S. 1997, MNRAS, 284, 465
Hui, X., Ford, H. C., Ciardullo, R., et al. 1993a, ApJ, 414, 463

Hui, X., Ford, H. C., Ciardullo, R., et al. 1993b, ApJS, 88, 423

Hui, X., Ford, H. C., Freeman, K. C., \& Dopita, M. A. 1995, ApJ, 449, 592

Israel, F. P. 1998, ARA\&A, 8, 237

Jacoby, G. H. 1989, ApJ, 339, 39

Jacoby, G. H., \& Ciardullo, R. 1999, ApJ, 515, 169 [JC99]

Jacoby, G. H., Branch, D., Ciardullo, R., et al. 1992, PASP, 104, 599

Jacoby, G. H., Morse, J. A., Fullton, L. K., et al. 1997, AJ, 114, 2611

Karakas, A., \& Lattanzio, J. C. 2003, PASA, 20, 393

Kingsburgh, R. L., \& Barlow, M. J. 1994, MNRAS, 271, 257

Kingsburgh, R. L., \& English, J. 1992, MNRAS, 259, 635

Leisy, P., \& Dennefeld, M. 2006, A\&A, 456, 451

Liu, X.-W., Storey, P. J., Barlow, M. J., et al. 2000, MNRAS, 312, 585

Magrini, L., Perinotto, M., Mampaso, A., et al. 2004, A\&A, 426, 779

Malin, D. A., Quinn, P., \& Graham, J. 1983, ApJ, 272, L5

Marigo, P., Girardi, L., Weiss, A., et al. 2004, A\&A, 423, 995

Marleau, F. R., Graham, J. R., Liu, M. C., et al. 2000, ApJ, 120, 1779

Matteucci, F., \& Recchi, S. 2001, ApJ, 558, 351

Méndez, R. H., Thomas, D., Saglia, R. P., et al. 2005, ApJ, 627, 767

Merrett, H. R., Merrifield, M. R., Douglas, N. G., et al. 2006, MNRAS, 369, 120

Minniti, D., \& Rejkuba, M. 2002, ApJ, 575, L59

Möllenhoff, C. 1981, A\&A, 99, 341

Nicholson, R., Bland-Hawthorn, J., \& Taylor, K. 1992, ApJ, 387, 503

Peimbert, M. 1978, in Planetary Nebulae, ed. Y. Terzian, IAU Symp., 76, 215

Peng, E. W., Ford, H. C., Freeman, K. C., et al. 2002, AJ, 124, 3144

Peng, E. W., Ford, H. C., \& Freeman, K. C. 2004, ApJ, 602, 685

Péquignot, D., Walsh, J. R., Zijlstra, A. A., et al. 2000, A\&A, 361, L1

Phillips, M. M. 1981, MNRAS, 197, 659

Quillen, A. C., Brookes, M. H., Keene, J., et al. 2006, ApJ, 645, 1092

Quillen, A. Bland-Hawthorn, J., Green, J. D., et al. 2008, MNRAS, 384, 1469

Rauch, T. 2003, A\&A, 403, 709

Rejkuba, M., Walsh, J. R. 2006, in Planetary Nebulae Beyond the Milky Way, eds. L. Stanghellini, J. R. Walsh, \& N. Douglas (Springer), 292

Rejkuba, M., Greggio, L., Harris, W. E., et al. 2005, ApJ, 631, 262

Rejkuba, M., Harris, W. E., Greggio, L., et al. 2011, A\&A, 526, A123

Richer, M. G. 1993, ApJ, 389, 27

Richer, M. G., \& McCall, M. L. 2008, ApJ, 684, 1190

Roth, M. M. 2006, in Science Perspectives for 3D Spectroscopy, eds. M. Kissler-Patig, J. R. Walsh, \& M. M. Roth, ESO Astrophysics Symp. (Berlin: Springer), 291

Schlegel, D. J., Finkbeiner, D. P., \& Davis, M. 1998, ApJ, 500, 525

Scott, P., Asplund, M., Grevesse, N. et al. 2009, ApJ, 691, L119

Seaton, M. J. 1979, MNRAS, 187, 73P

Stasińska, G., Richer, M., \& McCall, M. L. 1998, A\&A, 336, 667

Soria. R., Mould, J. R., Watson, A. M., et al. 1996, ApJ, 465, 79

Stasińska, G. 2002, Rev. Mex. Astron. Astrofis., 12, 62

Storey, P. J., \& Hummer, D. G. 1995, MNRAS, 272, 41

Thomas, D., Maraston, C., \& Bender, R., 2003, MNRAS, 339, 897

Tonry, J., L., Dressler, A., Blakeslee, J. P., et al. 2001, ApJ, 546, 681

Vassiliadis, E., \& Wood, P. R. 1994, ApJS, 92, 125

Walsh, J. R., Walton, N. J., Jacoby, G. H., \& Peletier, R. 1999, A\&A, 346, 753

Walsh, J. R., Jacoby, G. H., Peletier, R., et al. 2005, in Planetary Nebulae Beyond the Milky Way, eds. L. Stanghellini, J. R. Walsh, \& N. G. Douglas (Springer), 262

Woodley, K. A. 2006, AJ, 132, 2424

Woodley, K. A., Harris, W. E., Beasley, M. A., et al. 2007, AJ, 134, 494

Woodley, K. A., Gomez, M., Harris, W. E., et al. 2010, AJ, 139, 1871

Worthey, G., Faber, S. M., Gonzalez, J. J., \& Burstein, D. 1994, ApJS, 94, 687

Wright, N. J., Barlow, M. J., Ercolano, B., \& Rauch, T. 2011, MNRAS, 418, 370

Zhang, Y., Liu, X.-W., Luo, S.-G., et al. 2005, A\&A, 442, 249

Zoccali, M., Hill, V., Lecureur, A., et al. 2008, A\&A, 486, 177

Pages 16 to 28 are available in the electronic edition of the journal at http://www . aanda. org 
A\&A 544, A70 (2012)

Table 5. Summed PN spectra of three fields in NGC 5128 - Observed line fluxes.

\begin{tabular}{|c|c|c|c|c|c|c|c|}
\hline & & \multicolumn{2}{|c|}{ 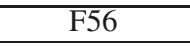 } & \multicolumn{2}{|c|}{$\overline{\mathrm{F} 42}$} & \multicolumn{2}{|c|}{$\bar{F}$ F34 } \\
\hline Species & $\lambda(\AA)$ & $F_{\mathrm{Obs}}$ & \pm & $F_{\text {Obs }}$ & \pm & $F_{\mathrm{Obs}}$ & \pm \\
\hline$[\mathrm{O}$ II] & 3727 & 28.6 & 1.7 & 23.9 & 2.2 & 54.5 & 2.9 \\
\hline [Ne III] & 3868 & 84.0 & 1.7 & 62.3 & 1.5 & 74.0 & 1.9 \\
\hline H I & 3889 & 17.3 & 4.4 & 13.3 & 1.6 & 14.3 & 1.2 \\
\hline$[\mathrm{Ne} \mathrm{III}]+\mathrm{H} \epsilon$ & 3970 & 33.5 & 1.0 & 26.9 & 1.5 & 34.4 & 1.9 \\
\hline $\mathrm{H} \delta$ & 4101 & 21.1 & 1.2 & 10.7 & 1.2 & 21.3 & 1.1 \\
\hline $\mathrm{H} \gamma$ & 4340 & 46.7 & 1.9 & 41.5 & 2.0 & 40.8 & 1.5 \\
\hline [O III] & 4363 & 8.7 & 0.7 & 6.1 & 1.3 & 8.5 & 0.6 \\
\hline $\mathrm{He} \mathrm{I}$ & 4471 & 8.0 & 1.0 & 6.5 & 1.0 & 5.0 & 3.3 \\
\hline He II & 4686 & 10.7 & 1.2 & 9.3 & 1.9 & 13.1 & 1.1 \\
\hline $\mathrm{H} \beta$ & 4861 & 100.0 & 0.0 & 100.0 & 0.0 & 100.0 & 0.0 \\
\hline [O III] & 4959 & 391.3 & 5.7 & 396.4 & 7.1 & 417.1 & 7.1 \\
\hline [O III] & 5007 & 1159.6 & 16.0 & 1213.6 & 20.2 & 1247.8 & 20.6 \\
\hline He I & 5016 & 3.7 & 0.4 & & & & \\
\hline$[\mathrm{N} \mathrm{I}]$ & 5199 & 3.2 & 0.2 & & & & \\
\hline $\mathrm{He} \mathrm{I}$ & 5876 & 14.5 & 1.9 & 29.9 & 5.6 & & \\
\hline$[\mathrm{N} \mathrm{II}]$ & 6548 & 50.3 & 1.2 & 62.7 & 4.4 & 62.7 & 4.6 \\
\hline $\mathrm{H} \alpha$ & 6562 & 390.6 & 3.8 & 422.7 & 9.8 & 412.8 & 14.7 \\
\hline [N II] & 6583 & 152.2 & 1.8 & 149.2 & 4.3 & 115.7 & 5.5 \\
\hline $\mathrm{He} \mathrm{I}$ & 6678 & 4.6 & 0.8 & 7.1 & 4.6 & & \\
\hline [S II] & 6716 & 4.5 & 0.4 & 7.3 & 2.8 & & \\
\hline [S II] & 6730 & 10.9 & 1.1 & 27.9 & 3.0 & & \\
\hline [Ar III] & 7133 & 28.5 & 1.1 & 22.9 & 3.1 & & \\
\hline$[\mathrm{O} \mathrm{II}]$ & 7325 & 27.2 & 2.4 & 19.6 & 4.6 & & \\
\hline $\log F(\mathrm{H} \beta)$ & & -15.46 & 0.01 & -15.42 & 0.01 & -15.78 & 0.01 \\
\hline$m_{5007 \mathrm{~A}}$ & & 22.24 & 0.03 & 22.10 & 0.04 & 22.98 & 0.04 \\
\hline
\end{tabular}

Table 6. NGC 5128 PN region spectra - Dereddened line fluxes.

\begin{tabular}{|c|c|c|c|c|c|c|c|}
\hline & & \multicolumn{2}{|c|}{$\overline{\overline{F 56}}$} & \multicolumn{2}{|c|}{$\overline{\bar{F} 42}$} & \multicolumn{2}{|c|}{$\overline{\bar{F} 34}$} \\
\hline Species & $\lambda(\AA)$ & $F_{\mathrm{Obs}}$ & \pm & $F_{\mathrm{Obs}}$ & \pm & $F_{\mathrm{Obs}}$ & \pm \\
\hline [O II] & 3727 & 37.0 & 2.3 & 33.0 & 3.0 & 73.8 & 3.9 \\
\hline$[\mathrm{Ne}$ III] & 3868 & 105.9 & 2.1 & 83.3 & 2.1 & 97.2 & 2.5 \\
\hline $\mathrm{H} \mathrm{I}$ & 3889 & 72.0 & 5.5 & 17.7 & 2.1 & 18.7 & 1.5 \\
\hline$[\mathrm{Ne} \mathrm{III}]+\mathrm{H} \epsilon$ & 3970 & 41.4 & 1.2 & 35.0 & 1.9 & 44.2 & 2.4 \\
\hline $\mathrm{H} \delta$ & 4101 & 25.4 & 1.4 & 13.5 & 1.4 & 26.4 & 1.4 \\
\hline $\mathrm{H} \gamma$ & 4340 & 53.1 & 1.8 & 48.7 & 2.4 & 47.4 & 1.7 \\
\hline [O III] & 4363 & 9.8 & 0.8 & 6.6 & 1.3 & 9.9 & 0.6 \\
\hline $\mathrm{He} \mathrm{I}$ & 4471 & 8.9 & 1.0 & 7.4 & 1.2 & 5.6 & 3.6 \\
\hline He II & 4686 & 11.2 & 1.3 & 9.8 & 2.0 & 13.8 & 1.1 \\
\hline $\mathrm{H} \beta$ & 4861 & 100.0 & 0.0 & 100.0 & 0.0 & 100.0 & 0.0 \\
\hline [O III] & 4959 & 381.8 & 5.6 & 384.6 & 6.9 & 405.5 & 6.9 \\
\hline [O III] & 5007 & 1118.3 & 15.4 & 1160.0 & 20.0 & 1196.3 & 19.7 \\
\hline $\mathrm{He} \mathrm{I}$ & 5016 & 3.6 & 0.4 & & & & \\
\hline$[\mathrm{N} \mathrm{I}]$ & 5199 & 3.0 & 0.2 & & & & \\
\hline $\mathrm{He} \mathrm{I}$ & 5876 & 11.6 & 1.5 & 22.8 & 4.3 & & \\
\hline [N II] & 6548 & 36.5 & 0.9 & 42.1 & 2.9 & 43.0 & 3.1 \\
\hline $\mathrm{H} \alpha$ & 6562 & 282.9 & 2.8 & 282.8 & 6.6 & 282.8 & 10.1 \\
\hline [N II] & 6583 & 109.9 & 1.3 & 99.4 & 2.9 & 79.0 & 3.7 \\
\hline $\mathrm{He} \mathrm{I}$ & 6678 & 3.3 & 0.5 & 4.7 & 3.0 & & \\
\hline [S II] & 6716 & 3.2 & 0.3 & 4.8 & 1.8 & & \\
\hline [S II] & 6730 & 7.7 & 0.8 & 18.1 & 1.9 & & \\
\hline [Ar III] & 7133 & 19.1 & 0.8 & 13.9 & 1.9 & & \\
\hline [O II] & 7325 & 17.9 & 1.6 & 11.6 & 2.7 & & \\
\hline & & 0.44 & 0.03 & 0.55 & 0.07 & 0.51 & 0.12 \\
\hline $\log F(\mathrm{H} \beta)$ & & -15.03 & 0.01 & -14.88 & 0.01 & -15.27 & 0.01 \\
\hline$m_{5007 \mathrm{~A}}$ & & 21.18 & 0.03 & 20.74 & 0.04 & 21.74 & 0.04 \\
\hline
\end{tabular}


J. R. Walsh et al.: Abundance distribution in NGC 5128 from PNe

Table 8. Cloudy photoionization modelling of 40 PN in NGC 5128.

\begin{tabular}{|c|c|c|c|c|c|c|c|c|c|c|}
\hline PN \# & $(\mathrm{He} / \mathrm{H})$ & $\begin{array}{c}\text { Elemen } \\
(\mathrm{N} / \mathrm{H})\end{array}$ & $\begin{array}{c}\text { Abun } \\
(\mathrm{O} / \mathrm{H})\end{array}$ & $\begin{array}{r}\text { ances } 12 \\
(\mathrm{Ne} / \mathrm{H})\end{array}$ & $\begin{array}{l}\log (\mathrm{A} / \mathrm{H} \\
(\mathrm{S} / \mathrm{H})^{1}\end{array}$ & $(\mathrm{Ar} / \mathrm{H})^{2}$ & $\begin{array}{c}T_{\text {eff }} \\
(\mathrm{kK})\end{array}$ & $\begin{array}{c}\log L \\
\left(L_{\odot}\right)\end{array}$ & $\begin{array}{l}\mathrm{Ne} \\
\left(\mathrm{cm}^{-3}\right)\end{array}$ & Quality $^{3}$ \\
\hline F56\#1 & 11.06 & 8.20 & 8.40 & 7.70 & 6.55 & 6.00 & 60 & 3.68 & 5000 & $\mathrm{~b}$ \\
\hline F56\#2 & 11.15 & 7.85 & 8.43 & 7.72 & & 6.00 & 155 & 4.20 & 10000 & $\mathrm{a}$ \\
\hline F56\#3 & 11.06 & 8.30 & 8.48 & 7.78 & 6.55 & 6.00 & 64 & 3.66 & 2000 & $\mathrm{~b}$ \\
\hline F56\#4 & 11.06 & 8.30 & 8.38 & 7.70 & 6.55 & 6.50 & 78 & 3.75 & 5000 & $\mathrm{~b}$ \\
\hline F56\#5 & 11.06 & 7.40 & 8.55 & 7.85 & & 6.35 & 150 & 3.74 & 10000 & c \\
\hline F56\#6 & 11.20 & 8.30 & 8.64 & 7.95 & & 6.60 & 75 & 4.15 & 6000 & b \\
\hline F56\#8 & 11.20 & 8.62 & 8.60 & 7.80 & 6.80 & 6.50 & 63 & 3.77 & 9000 & $\mathrm{~b}$ \\
\hline F56\#10 & 11.10 & 8.20 & 8.38 & 7.60 & 6.55 & 6.60 & 88 & 4.03 & 15000 & $\mathrm{~b}$ \\
\hline F56\#11 & 10.90 & 8.42 & 8.54 & 7.70 & 7.00 & 6.40 & 90 & 3.67 & 22500 & $\mathrm{a}$ \\
\hline F56\#12a & 11.10 & 8.53 & 8.80 & 8.10 & 6.55 & 6.40 & 200 & 3.86 & 10000 & $\mathrm{a}$ \\
\hline F56\#12b & 11.00 & 8.80 & 8.55 & 7.85 & & 6.50 & 45 & 3.93 & 35000 & $\mathrm{~b}$ \\
\hline F56\#13b & 11.00 & 7.60 & 8.52 & 7.70 & & 6.20 & 105 & 3.76 & 20000 & $\mathrm{a}$ \\
\hline F56\#14 & 11.06 & 8.40 & 8.46 & 7.70 & 6.55 & 6.30 & 95 & 3.86 & 15000 & $\mathrm{~b}$ \\
\hline F56\#15 & 11.06 & 7.90 & 8.47 & 7.70 & 6.55 & 6.20 & 70 & 3.86 & 10000 & $\mathrm{c}$ \\
\hline F56\#16 & 11.06 & 8.20 & 8.47 & 7.60 & 6.55 & 6.20 & 70 & 3.91 & 10000 & $\mathrm{~b}$ \\
\hline F56\#18 & 11.06 & 8.63 & 8.51 & 7.85 & 6.90 & & 90 & 3.87 & 15000 & b \\
\hline F42\#1 & 11.12 & 7.85 & 8.45 & 7.65 & 6.90 & 6.20 & 80 & 3.83 & 10000 & $\mathrm{c}$ \\
\hline F42\#2 & 11.06 & 8.19 & 8.68 & 8.03 & & & 155 & 4.05 & 10000 & $\mathrm{a}$ \\
\hline F42\#3 & 11.06 & 8.26 & 8.44 & 7.50 & & 6.40 & 50 & 3.99 & 10000 & $\mathrm{c}$ \\
\hline F42\#4 & 11.01 & 8.12 & 8.03 & 7.10 & 6.40 & 6.20 & 86 & 4.00 & 12000 & $\mathrm{a}$ \\
\hline F42\#6 & 11.15 & 7.30 & 8.44 & 7.60 & 7.10 & & 63 & 3.63 & 13000 & $\mathrm{~b}$ \\
\hline F42\#7 & 11.06 & 8.00 & 8.51 & 7.70 & & & 127 & 3.64 & 10000 & $\mathrm{~b}$ \\
\hline F42\#8 & 11.06 & 8.20 & 8.46 & 7.70 & & & 93 & 3.86 & 15000 & $\mathrm{~b}$ \\
\hline F42\#9 & 11.12 & 8.20 & 8.45 & 7.70 & & 6.50 & 74 & 3.78 & 30000 & b \\
\hline F42\#10 & 11.28 & 8.18 & 8.10 & 7.40 & 6.85 & 6.10 & 120 & 4.29 & 2500 & a \\
\hline F42\#11 & 11.06 & 8.25 & 8.28 & 7.35 & 6.85 & 6.20 & 100 & 3.72 & 10000 & $\mathrm{c}$ \\
\hline $\mathrm{F} 42 \# 12 \mathrm{~b}$ & 11.06 & 8.22 & 8.69 & 8.07 & & & 108 & 4.09 & 10000 & $\mathrm{~b}$ \\
\hline F42\#13 & 11.06 & 8.28 & 8.90 & 8.25 & & & 190 & 3.84 & 16000 & $\mathrm{a}$ \\
\hline F42\#14b & 11.06 & 8.20 & 9.00 & 8.40 & 6.70 & & 220 & 4.06 & 20000 & $\mathrm{a}$ \\
\hline F42\#16a & 11.06 & 8.28 & 8.72 & 7.92 & & & 100 & 3.78 & 14000 & $\mathrm{a}$ \\
\hline F42-18 & 11.06 & 7.68 & 8.49 & 7.45 & & & 120 & 4.35 & 10000 & $\mathrm{c}$ \\
\hline F34\#1 & 11.06 & 8.25 & 8.73 & 8.00 & & 5.65 & 71 & 3.89 & 8500 & $\mathrm{a}$ \\
\hline F34\#2 & 11.23 & 7.62 & 8.09 & 7.38 & & & 90 & 4.11 & 1500 & $\mathrm{a}$ \\
\hline F34\#4 & 11.06 & 8.10 & 8.46 & 7.70 & & 6.30 & 67 & 3.90 & 8000 & b \\
\hline F34\#7 & 11.06 & 8.30 & 8.91 & 8.30 & & 6.50 & 82 & 3.99 & 13000 & $\mathrm{a}$ \\
\hline F34\#11 & 11.16 & 7.40 & 8.53 & 7.78 & & & 180 & 3.59 & 30000 & $\mathrm{a}$ \\
\hline F34\#12 & 11.16 & 7.35 & 8.48 & 7.30 & & & 167 & 3.13 & 30000 & $\mathrm{a}$ \\
\hline F34\#14 & 11.06 & 7.90 & 8.62 & 7.82 & & & 70 & 3.61 & 20000 & $\mathrm{~b}$ \\
\hline F34\#15 & 11.06 & 7.40 & 8.43 & 7.76 & & & 123 & 3.90 & 10000 & b \\
\hline F34\#16 & 11.06 & 8.28 & 8.63 & 7.95 & 6.55 & & 180 & 3.70 & 5000 & $\mathrm{a}$ \\
\hline
\end{tabular}

Notes. ${ }^{(1)} 12+\log (\mathrm{S} / \mathrm{H})$ listed if default value of 6.55 not applied. ${ }^{(2)} 12+\log (\mathrm{Ar} / \mathrm{H})$ listed if default value of 6.00 not applied. ${ }^{(3)}$ Quality. a: He II and [O II] both detected; b: [O II] but not He II detected, or He II detected but not [O II]; c: neither He II nor [O II] detected. 
Table 9. Comparative Cloudy photoionization modelling of F56\#2.

\begin{tabular}{|c|c|c|c|c|c|c|c|c|c|c|}
\hline Species & $\lambda(\AA)$ & $\begin{array}{l}\text { Flux \& } \\
\text { error }\end{array}$ & Model & $\begin{array}{c}\text { Model } \\
\mathrm{O}+0.2 \\
\end{array}$ & $\begin{array}{l}\text { Model } \\
\mathrm{O}-0.2\end{array}$ & $\begin{array}{c}\text { Model } \\
T_{\mathrm{BB}}+20 \mathrm{kK}\end{array}$ & $\begin{array}{c}\text { Model } \\
T_{\mathrm{BB}}-20 \mathrm{kK}\end{array}$ & $\begin{array}{c}\text { Model } \\
N_{\mathrm{e}} \times 2.0\end{array}$ & $\begin{array}{c}\text { Model } \\
N_{\mathrm{e}} \times 0.5\end{array}$ & $\begin{array}{c}\text { Model } \\
\text { Mod. atmos. }\end{array}$ \\
\hline [O II] & 3727 & $56 \pm 13$ & 59 & 58 & 47 & 53 & 59 & 32 & 53 & 57 \\
\hline$[\mathrm{Ne} I \mathrm{II}]$ & 3868 & $124 \pm 13$ & 124 & 127 & 128 & 122 & 123 & 126 & 125 & 125 \\
\hline [O III] & 4363 & $28 \pm 8$ & 24 & 15 & 27 & 26 & 22 & 22 & 22 & 27 \\
\hline $\mathrm{He} \mathrm{I}$ & 4471 & $6 \pm 3$ & 6 & 6 & 6 & 3 & 11 & 6 & 9 & 6 \\
\hline He II & 4686 & $39 \pm 8$ & 39 & 38 & 34 & 46 & 30 & 37 & 30 & 38 \\
\hline $\mathrm{H} \beta$ & 4861 & $100 \pm 0$ & 100 & 100 & 100 & 100 & 100 & 100 & 100 & 100 \\
\hline [O III] & 4959 & $457 \pm 31$ & 449 & 448 & 398 & 451 & 471 & 454 & 458 & 456 \\
\hline [O III] & 5007 & $1367 \pm 90$ & 1350 & 1348 & 1199 & 1358 & 1417 & 1367 & 1377 & 1371 \\
\hline $\mathrm{He} \mathrm{I}$ & 5876 & $6 \pm 3$ & 18 & 18 & 19 & 11 & 33 & 19 & 28 & 20 \\
\hline $\mathrm{H} \alpha$ & 6562 & $281 \pm 19$ & 286 & 285 & 290 & 286 & 286 & 284 & 285 & 288 \\
\hline$[\mathrm{N} \mathrm{II}]$ & 6583 & $55 \pm 5$ & 54 & 57 & 58 & 53 & 53 & 54 & 53 & 57 \\
\hline $\mathrm{He} \mathrm{I}$ & 6678 & $7 \pm 4$ & 4 & 5 & 4 & 2 & 8 & 4 & 7 & 5 \\
\hline [Ar III] & 7133 & $8 \pm 3$ & 9 & 6 & 10 & 8 & 9 & 7 & 9 & 8 \\
\hline [O II] & 7325 & $14 \pm 4$ & 16 & 14 & 13 & 19 & 14 & 15 & 9 & 16 \\
\hline \multicolumn{11}{|l|}{ Model input } \\
\hline$T_{\mathrm{BB}}(\mathrm{kK})$ & & & 155 & 152.5 & 150 & 175 & 135 & 150 & 132.5 & $170^{\top}$ \\
\hline$N_{\mathrm{H}}\left(\mathrm{cm}^{-3}\right)$ & & & 10000 & 10000 & 10000 & 12000 & 8000 & 20000 & 5000 & 10000 \\
\hline $12+\log (\mathrm{He})$ & & & 11.15 & 11.15 & 11.20 & 11.00 & 11.35 & 11.15 & 11.30 & 11.18 \\
\hline $12+\log (\mathrm{C})$ & & & 8.40 & 9.20 & 6.60 & 8.40 & 8.40 & 8.70 & 8.40 & 8.20 \\
\hline $12+\log (\mathrm{N})$ & & & 7.85 & 8.02 & 7.84 & 7.85 & 7.88 & 8.01 & 8.05 & 7.85 \\
\hline $12+\log (\mathrm{O})$ & & & 8.43 & 8.63 & 8.23 & 8.48 & 8.43 & 8.49 & 8.40 & 8.39 \\
\hline $12+\log (\mathrm{Ne})$ & & & 7.72 & 7.96 & 7.57 & 7.75 & 7.70 & 7.78 & 7.70 & 7.67 \\
\hline $12+\log (\mathrm{Ar})$ & & & 6.00 & 6.00 & 6.15 & 6.00 & 6.00 & 6.00 & 6.00 & 5.95 \\
\hline FoM & & & 7.53 & 8.30 & 13.46 & 7.34 & 14.79 & 9.06 & 12.90 & 8.56 \\
\hline
\end{tabular}

Notes. ${ }^{(1)}$ Model atmosphere: PG1159 atmosphere with $T_{*}=170000 \mathrm{~K}$ and $\log g=7.00$ from Rauch (2003). S abundance fixed at $12+$ $\log (\mathrm{S} / \mathrm{H})=6.55$.

Table 10. Comparative Cloudy photoionization modelling of F34\#1.

\begin{tabular}{|c|c|c|c|c|c|c|c|c|c|c|}
\hline Species & $\lambda(\AA)$ & $\begin{array}{l}\text { Flux \& } \\
\text { error }\end{array}$ & Model & $\begin{array}{c}\text { Model } \\
\mathrm{O}+0.2\end{array}$ & $\begin{array}{l}\text { Model } \\
\mathrm{O}-0.2\end{array}$ & $\begin{array}{c}\text { Model } \\
T_{\mathrm{BB}}+10 \mathrm{kK}\end{array}$ & $\begin{array}{c}\text { Model } \\
T_{\mathrm{BB}}-10 \mathrm{kK}\end{array}$ & $\begin{array}{c}\text { Model } \\
N_{\mathrm{e}} \times 2.0\end{array}$ & $\begin{array}{c}\text { Model } \\
N_{\mathrm{e}} \times 0.5\end{array}$ & $\begin{array}{l}\text { Model } \\
\text { Mod. atmos. }\end{array}$ \\
\hline [O II] & 3727 & $86 \pm 9$ & 89 & 85 & 88 & 87 & 60 & 49 & 144 & 85 \\
\hline$[\mathrm{Ne}$ III $]$ & 3868 & $93 \pm 6$ & 91 & 92 & 93 & 92 & 95 & 92 & 94 & 96 \\
\hline He II & 4686 & $2 \pm 2$ & 2 & 2 & 4 & 4 & 1 & 2 & 4 & 0 \\
\hline $\mathrm{H} \beta$ & 4861 & $100 \pm 0$ & 100 & 100 & 100 & 100 & 100 & 100 & 100 & 100 \\
\hline [O III] & 4959 & $393 \pm 15$ & 384 & 391 & 384 & 393 & 248 & 385 & 387 & 390 \\
\hline [O III] & 5007 & $1164 \pm 41$ & 1156 & 1177 & 1157 & 1183 & 747 & 1157 & 1166 & 1174 \\
\hline $\mathrm{H} \alpha$ & 6562 & $283 \pm 12$ & 288 & 290 & 287 & 286 & 286 & 287 & 288 & 287 \\
\hline$[\mathrm{N} \mathrm{II}]$ & 6583 & $104 \pm 6$ & 104 & 104 & 104 & 104 & 103 & 104 & 105 & 103 \\
\hline [Ar III] & 7133 & $4 \pm 3$ & 3 & 4 & 4 & 4 & 3 & 3 & 4 & 4 \\
\hline [O II] & 7325 & $4 \pm 3$ & 17 & 16 & 17 & 17 & 8 & 16 & 17 & 14 \\
\hline \multicolumn{11}{|l|}{ Model input } \\
\hline$T_{\mathrm{BB}}(\mathrm{kK})$ & & & 71 & 68 & 78 & 81 & 61 & 67.5 & 80 & $70^{1}$ \\
\hline$N_{H}\left(\mathrm{~cm}^{-3}\right)$ & & & 8500 & 10000 & 7500 & 8500 & 8500 & 17000 & 4250 & 7000 \\
\hline $12+\log (\mathrm{He} / \mathrm{H})$ & & & 11.06 & 11.06 & 11.06 & 10.90 & 11.30 & 11.06 & 10.90 & 11.20 \\
\hline $12+\log (\mathrm{C})$ & & & 8.60 & 8.50 & 8.40 & 8.80 & 8.50 & 8.60 & 8.30 & 8.20 \\
\hline $12+\log (\mathrm{N})$ & & & 8.25 & 8.38 & 8.13 & 8.16 & 8.50 & 8.40 & 8.03 & 8.18 \\
\hline $12+\log (\mathrm{O})$ & & & 8.73 & 8.93 & 8.53 & 8.60 & 9.00 & 8.78 & 8.54 & 8.60 \\
\hline $12+\log (\mathrm{Ne})$ & & & 8.00 & 8.23 & 7.78 & 7.84 & 8.60 & 8.07 & 7.77 & 7.88 \\
\hline $12+\log (\mathrm{Ar})$ & & & 5.65 & 5.85 & 5.55 & 5.60 & 5.90 & 6.65 & 5.55 & 5.60 \\
\hline FoM & & & 6.44 & 5.79 & 6.48 & 6.74 & 26.62 & 10.20 & 12.90 & 5.30 \\
\hline
\end{tabular}

Notes. ${ }^{(1)}$ Model atmosphere: PG1159 atmosphere with $T_{*}=70000 \mathrm{~K}$ and $\log g=7.00$ from Rauch (2003). S abundance fixed at $12+$ $\log (\mathrm{S} / \mathrm{H})=6.55$. 
J. R. Walsh et al.: Abundance distribution in NGC 5128 from PNe

Table 11. NGC 5128 PN spectra - observed line fluxes.

\begin{tabular}{|c|c|c|c|c|c|c|c|c|c|c|c|}
\hline \multirow[b]{2}{*}{ Species } & \multirow[b]{2}{*}{$\lambda(\AA)$} & \multicolumn{2}{|c|}{$\begin{array}{c}\text { F56 \#1 } \\
5615 \\
(+297.6,-492.5) \\
\end{array}$} & \multicolumn{2}{|c|}{$\begin{array}{c}\text { F56 \#2 } \\
5601 \\
(+284.3,-465.9) \\
\end{array}$} & \multicolumn{2}{|c|}{$\begin{array}{c}\text { F56 \#3 } \\
5621 \\
(+287.4,-448.7) \\
\end{array}$} & \multicolumn{2}{|c|}{$\begin{array}{c}\text { F56 \#4 } \\
5611 \\
(+298.7,-405.0) \\
\end{array}$} & \multicolumn{2}{|c|}{$\begin{array}{c}\text { F56 \#5 } \\
5608 \\
(+268.9,-394.1) \\
\end{array}$} \\
\hline & & $F_{\mathrm{Obs}}$ & \pm & $F_{\text {Obs }}$ & \pm & $F_{\text {Obs }}$ & \pm & $F_{\mathrm{Obs}}$ & \pm & $F_{\text {Obs }}$ & \pm \\
\hline$[\mathrm{O} \mathrm{II}]$ & 3727 & 38 & 32 & 43 & 10 & 84 & 20 & & & & \\
\hline [Ne III] & 3868 & 38 & 14 & 97 & 10 & 55 & 16 & 72 & 19 & 112 & 60 \\
\hline$[\mathrm{Ne} \mathrm{III}]+\mathrm{H} \epsilon$ & 3970 & & & 29 & 6 & & & & & & \\
\hline $\mathrm{H} \delta$ & 4101 & & & 22 & 8 & & & & & & \\
\hline $\mathrm{H} \gamma$ & 4340 & 36 & 13 & 48 & 9 & 43 & 14 & 31 & 13 & 46 & 48 \\
\hline [O III] & 4363 & & & 24 & 7 & & & & & & \\
\hline He I & 4471 & & & 5 & 3 & & & & & & \\
\hline $\mathrm{He}$ II & 4686 & & & 37 & 7 & & & & & & \\
\hline $\mathrm{H} \beta$ & 4861 & 100 & 0 & 100 & 0 & 100 & 0 & 100 & 0 & 100 & 0 \\
\hline [O III] & 4959 & 298 & 27 & 469 & 32 & 276 & 35 & 332 & 49 & 549 & 64 \\
\hline [O III] & 5007 & 867 & 69 & 1421 & 93 & 812 & 92 & 965 & 135 & 1606 & 174 \\
\hline He I & 5876 & & & 8 & 4 & & & 24 & 7 & & \\
\hline$[\mathrm{N} \mathrm{II}]$ & 6548 & 44 & 7 & 23 & 6 & 72 & 15 & 74 & 14 & & \\
\hline $\mathrm{H} \alpha$ & 6562 & 392 & 32 & 396 & 26 & 440 & 51 & 412 & 58 & 420 & 47 \\
\hline$[\mathrm{N}$ II $]$ & 6583 & 147 & 14 & 78 & 7 & 284 & 34 & 246 & 35 & 25 & 10 \\
\hline He I & 6678 & & & 10 & 6 & & & & & & \\
\hline [S II $]$ & 6716 & & & & & 39 & 30 & 21 & 8 & & \\
\hline [S II $]$ & 6730 & 13 & 7 & & & 20 & 11 & 11 & 6 & & \\
\hline [Ar III] & 7133 & 17 & 10 & 12 & 4 & 21 & 11 & 59 & 13 & 39 & 34 \\
\hline [O II] & 7325 & & & 22 & 6 & 104 & 35 & 28 & 12 & & \\
\hline $\log F(\mathrm{H} \beta)$ & & -16.37 & 0.03 & -16.06 & 0.03 & -16.50 & 0.05 & -16.39 & 0.06 & -16.59 & 0.05 \\
\hline \multirow[t]{2}{*}{$m_{5007 \mathrm{~A}}$} & & 24.84 & 0.09 & 23.52 & 0.07 & 25.22 & 0.12 & 24.76 & 0.15 & 24.72 & 0.12 \\
\hline & & \multicolumn{2}{|c|}{$\begin{array}{c}\text { F56 \#6 } \\
5602 \\
(+247.7,-372.5) \\
\end{array}$} & \multicolumn{2}{|c|}{$\begin{array}{c}\text { F56 \#8 } \\
5425 \\
(+260.2,-326.0) \\
\end{array}$} & \multicolumn{2}{|c|}{$\begin{array}{c}\text { F56 \#9 } \\
5456 \\
(+235.0,-312.2) \\
\end{array}$} & \multicolumn{2}{|c|}{$\begin{array}{c}\text { F56 \#10 } \\
5416 \\
(+241.5,-281.6) \\
\end{array}$} & \multicolumn{2}{|c|}{$\begin{array}{c}\text { F56\#11 } \\
5409 \\
(+240.2,-279.3) \\
\end{array}$} \\
\hline Species & $\lambda(\AA)$ & $F_{\text {Obs }}$ & \pm & $F_{\text {Obs }}$ & \pm & $F_{\mathrm{Obs}}$ & \pm & $F_{\text {Obs }}$ & \pm & $F_{\mathrm{Obs}}$ & \pm \\
\hline$[\mathrm{O} \mathrm{II}]$ & 3727 & 42 & 9 & 38 & 10 & & & 24 & 15 & 29 & 5 \\
\hline [Ne III] & 3868 & 75 & 12 & 49 & 13 & & & 69 & 11 & 96 & 9 \\
\hline$[\mathrm{Ne} \mathrm{III}]+\mathrm{H} \epsilon$ & 3970 & 52 & 8 & 31 & 16 & & & 53 & 31 & 47 & 23 \\
\hline $\mathrm{H} \delta$ & 4101 & 13 & 10 & 27 & 18 & & & 27 & 7 & 27 & 10 \\
\hline $\mathrm{H} \gamma$ & 4340 & 43 & 7 & 42 & 10 & & & 43 & 8 & 48 & 9 \\
\hline [O III] & 4363 & & & & & & & & & 15 & 4 \\
\hline He I & 4471 & & & & & & & & & & \\
\hline He II & 4686 & & & & & & & & & & \\
\hline $\mathrm{H} \beta$ & 4861 & 100 & 0 & 100 & 0 & 100 & 0 & 100 & 0 & 100 & 0 \\
\hline [O III] & 4959 & 432 & 35 & 292 & 27 & 263 & 108 & 382 & 29 & 476 & 37 \\
\hline [O III] & 5007 & 1273 & 100 & 865 & 78 & 794 & 311 & 1179 & 87 & 1370 & 103 \\
\hline $\mathrm{He} \mathrm{I}$ & 5876 & 14 & 3 & 33 & 8 & & & 26 & 8 & 11 & 7 \\
\hline [N II] & 6548 & 39 & 5 & 76 & 9 & & & 29 & 5 & 59 & 11 \\
\hline $\mathrm{H} \alpha$ & 6562 & 468 & 37 & 362 & 33 & 301 & 123 & 388 & 29 & 336 & 77 \\
\hline$[\mathrm{N} \mathrm{II}]$ & 6583 & 149 & 13 & 233 & 22 & 51 & 36 & 113 & 10 & 161 & 35 \\
\hline $\mathrm{He} \mathrm{I}$ & 6678 & 16 & 5 & 18 & 11 & & & & & & \\
\hline [S II] & 6716 & & & 13 & 4 & & & & & & \\
\hline [S II] & 6730 & & & 19 & 5 & & & 12 & 6 & 10 & 3 \\
\hline [Ar III] & 7133 & 56 & 9 & 39 & 8 & & & 51 & 8 & 31 & 7 \\
\hline [O II] & 7325 & 29 & 12 & 46 & 16 & & & 25 & 19 & & \\
\hline $\log F(\mathrm{H} \beta)$ & & -16.08 & 0.03 & -16.13 & 0.04 & -17.37 & 0.19 & -16.14 & 0.03 & -16.12 & 0.03 \\
\hline \multirow[t]{2}{*}{$m_{5007 \mathrm{~A}}$} & & 23.70 & 0.09 & 24.23 & 0.10 & 27.43 & 0.48 & 23.93 & 0.08 & 23.71 & 0.08 \\
\hline & & \multicolumn{2}{|c|}{$\begin{array}{c}\text { F56 \#12a } \\
5418 \\
(+221.9,-245.1)\end{array}$} & \multicolumn{2}{|c|}{$\begin{array}{c}\text { F56\#12b } \\
5438 \\
(+220.2,-242.0) \\
\end{array}$} & $\begin{array}{r}\text { F56 } \\
5 \\
(+201.5 \\
\end{array}$ & $\begin{array}{l}13 \mathrm{~b} \\
3 \\
225.7) \\
\end{array}$ & $\begin{array}{r}\mathrm{F} 5 \\
5 \\
(+183 . \\
\end{array}$ & $\begin{array}{l}14 \\
217.4) \\
\end{array}$ & $\begin{array}{r}\mathrm{F} 5 \\
5 \\
(+169.1 \\
\end{array}$ & $\begin{array}{l}15 \\
200.2) \\
\end{array}$ \\
\hline Species & $\lambda(\AA)$ & $F_{\text {Obs }}$ & \pm & $F_{\text {Obs }}$ & \pm & $F_{\text {Obs }}$ & \pm & $F_{\mathrm{Obs}}$ & \pm & $F_{\text {Obs }}$ & \pm \\
\hline$[\mathrm{O}$ II] & 3727 & 77 & 52 & 15 & 11 & 31 & 12 & 28 & 11 & & \\
\hline$[\mathrm{Ne} \mathrm{III}]$ & 3868 & 129 & 27 & 32 & 11 & 82 & 14 & 84 & 11 & 55 & 12 \\
\hline$[\mathrm{Ne} \mathrm{III}]+\mathrm{H} \epsilon$ & 3970 & 66 & 49 & 27 & 14 & 40 & 13 & 50 & 40 & & \\
\hline $\mathrm{H} \delta$ & 4101 & & & 15 & 8 & & & 26 & 9 & 26 & 13 \\
\hline
\end{tabular}


Table 11. continued.

\begin{tabular}{|c|c|c|c|c|c|c|c|c|c|c|c|}
\hline $\mathrm{H} \gamma$ & 4340 & \multirow[t]{3}{*}{41} & \multirow[t]{3}{*}{13} & \multirow[t]{3}{*}{36} & \multirow[t]{3}{*}{9} & \multirow[t]{3}{*}{40} & \multirow[t]{3}{*}{14} & \multirow[t]{4}{*}{30} & \multirow[t]{4}{*}{11} & \multirow[t]{4}{*}{ 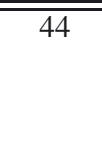 } & \multirow[t]{3}{*}{13} \\
\hline [O III] & 4363 & & & & & & & & & & \\
\hline He I & 4471 & & & & & & & & & & \\
\hline He II & 4686 & 57 & 13 & & & 13 & 11 & & & & \\
\hline $\mathrm{H} \beta$ & 4861 & 100 & 0 & 100 & 0 & 100 & 0 & 100 & 0 & 100 & 0 \\
\hline [O III] & 4959 & 684 & 97 & 180 & 20 & 529 & 50 & 445 & 41 & 366 & 45 \\
\hline [O III] & 5007 & 1956 & 271 & 516 & 51 & 1540 & 140 & 1336 & 121 & 1083 & 128 \\
\hline $\mathrm{He} \mathrm{I}$ & 5876 & 20 & 11 & 16 & 8 & 15 & 6 & 23 & 6 & & \\
\hline [N II] & 6548 & 111 & 19 & 77 & 9 & & & 67 & 9 & 24 & 8 \\
\hline $\mathrm{H} \alpha$ & 6562 & 420 & 59 & 365 & 36 & 383 & 36 & 382 & 35 & 415 & 49 \\
\hline$[\mathrm{N} \mathrm{II}]$ & 6583 & 335 & 48 & 266 & 26 & 34 & 7 & 194 & 19 & 63 & 10 \\
\hline He I & 6678 & & & & & 11 & 4 & & & & \\
\hline [S II] & 6716 & & & & & & & & & & \\
\hline [S II] & 6730 & 12 & 9 & & & & & 11 & 5 & 20 & 8 \\
\hline [Ar III] & 7133 & 31 & 8 & 32 & 7 & 19 & 8 & 33 & 7 & 21 & 8 \\
\hline$[\mathrm{O} \mathrm{II}]$ & 7325 & 69 & 20 & 20 & 12 & & & 58 & 15 & & \\
\hline $\log F(\mathrm{H} \beta)$ & & -16.37 & 0.06 & -16.17 & 0.04 & -16.22 & 0.04 & -16.11 & 0.04 & -16.21 & 0.05 \\
\hline \multirow{2}{*}{$m_{5007 \mathrm{~A}}$} & & 23.97 & 0.15 & 24.91 & 0.11 & 23.83 & 0.10 & 23.71 & 0.10 & 24.19 & 0.13 \\
\hline & & \multicolumn{2}{|c|}{$\begin{array}{c}\text { F56 \#16 } \\
5419 \\
(+139.3,-175.8) \\
\end{array}$} & \multicolumn{2}{|c|}{$\begin{array}{c}\text { F56 \#17 } \\
5428 \\
(+146.7,-145.7) \\
\end{array}$} & \multicolumn{2}{|c|}{$\begin{array}{c}\text { F56 \#18 } \\
5422 \\
(+107.6,-151.4) \\
\end{array}$} & & & & \\
\hline Species & $\lambda(\AA)$ & $F_{\mathrm{Obs}}$ & \pm & $F_{\mathrm{Obs}}$ & \pm & $F_{\mathrm{Obs}}$ & \pm & & & & \\
\hline [O II] & 3727 & 41 & 16 & & & 26 & 18 & & & & \\
\hline$[\mathrm{Ne} \mathrm{III}]$ & 3868 & 48 & 12 & 26 & 10 & 88 & 21 & & & & \\
\hline$[\mathrm{Ne} \mathrm{III}]+\mathrm{H} \epsilon$ & 3970 & 16 & 7 & 18 & 10 & 29 & 15 & & & & \\
\hline $\mathrm{H} \delta$ & 4101 & & & & & & & & & & \\
\hline $\mathrm{H} \gamma$ & 4340 & 37 & 7 & 30 & 8 & & & & & & \\
\hline [O III] & 4363 & & & & & & & & & & \\
\hline He I & 4471 & 20 & 7 & & & & & & & & \\
\hline $\mathrm{He}$ II & 4686 & & & & & & & & & & \\
\hline $\mathrm{H} \beta$ & 4861 & 100 & 0 & 100 & 0 & 100 & 0 & & & & \\
\hline [O III] & 4959 & 369 & 31 & 275 & 34 & 416 & 74 & & & & \\
\hline [O III] & 5007 & 1063 & 84 & 861 & 101 & 1315 & 230 & & & & \\
\hline He I & 5876 & 24 & 9 & 30 & 12 & 28 & 13 & & & & \\
\hline$[\mathrm{N} \mathrm{III}]$ & 6548 & 36 & 7 & & & 102 & 23 & & & & \\
\hline $\mathrm{H} \alpha$ & 6562 & 415 & 34 & 356 & 42 & 447 & 79 & & & & \\
\hline [N II] & 6583 & 147 & 14 & & & 323 & 58 & & & & \\
\hline $\mathrm{He} \mathrm{I}$ & 6678 & & & & & & & & & & \\
\hline [S II] & 6716 & & & & & & & & & & \\
\hline [S II $]$ & 6730 & 11 & 6 & & & 30 & 11 & & & & \\
\hline [Ar III] & 7133 & 24 & 8 & & & & & & & & \\
\hline$[\mathrm{O} \mathrm{II}]$ & 7325 & & & & & & & & & & \\
\hline $\log F(\mathrm{H} \beta)$ & & -16.15 & 0.03 & -16.19 & 0.05 & -16.35 & 0.08 & & & & \\
\hline \multirow[t]{2}{*}{$m_{5007 \mathrm{~A}}$} & & 24.07 & 0.09 & 24.41 & 0.13 & 24.34 & 0.19 & & & & \\
\hline & & $\begin{array}{r}\mathrm{F} 4 \\
4 \\
(-393 \\
\end{array}$ & $\begin{array}{l}1 \\
-84.9) \\
\end{array}$ & $\begin{array}{r}\mathrm{F} 4 \\
4 \\
(-362 . \\
\end{array}$ & $\begin{array}{l}2 \\
2 \\
-71.3) \\
\end{array}$ & $\begin{array}{r}\mathrm{F} 4 \\
4 \\
(-345.6 \\
\end{array}$ & $\begin{array}{l}83 \\
726.0) \\
\end{array}$ & $\begin{array}{r}\mathrm{F} \\
4 \\
(-318 \\
\end{array}$ & $\begin{array}{l}4 \\
79.3) \\
\end{array}$ & $\begin{array}{r}\mathrm{F} 4 \\
0 \\
(-278 \\
\end{array}$ & $\begin{array}{l}6 \\
34.0) \\
\end{array}$ \\
\hline Species & $\lambda(\AA)$ & $F_{\mathrm{Obs}}$ & \pm & $F_{\text {Obs }}$ & \pm & $F_{\mathrm{Obs}}$ & \pm & $F_{\mathrm{Obs}}$ & \pm & $F_{\text {Obs }}$ & \pm \\
\hline$[\mathrm{O} \mathrm{II}]$ & 3727 & & & 75 & 37 & & & 21 & 6 & 44 & 34 \\
\hline$[\mathrm{Ne} \mathrm{III]}]$ & 3868 & 61 & 8 & 134 & 17 & 19 & 4 & 28 & 4 & 42 & 7 \\
\hline$[\mathrm{Ne} \mathrm{III}]+\mathrm{H} \epsilon$ & 3970 & 33 & 6 & 51 & 8 & & & 20 & 3 & & \\
\hline $\mathrm{H} \delta$ & 4101 & 17 & 5 & & & 19 & 10 & 17 & 4 & & \\
\hline $\mathrm{H} \gamma$ & 4340 & 37 & 6 & 45 & 29 & 43 & 7 & 42 & 5 & 37 & 7 \\
\hline [O III] & 4363 & & & & & & & & & & \\
\hline $\mathrm{He} \mathrm{I}$ & 4471 & & & & & & & 5 & 2 & & \\
\hline $\mathrm{He}$ II & 4686 & & & 36 & 8 & & & & & & \\
\hline $\mathrm{H} \beta$ & 4861 & 100 & 0 & 100 & 0 & 100 & 0 & 100 & 0 & 100 & 0 \\
\hline [O III] & 4959 & 350 & 24 & 625 & 60 & 182 & 13 & 222 & 12 & 289 & 25 \\
\hline [O III] & 5007 & 1078 & 70 & 1872 & 177 & 582 & 38 & 663 & 33 & 778 & 64 \\
\hline $\mathrm{He} \mathrm{I}$ & 5876 & 28 & 8 & & & & & 7 & 2 & 32 & 11 \\
\hline$[\mathrm{N} \mathrm{II}]$ & 6548 & & & & & 65 & 9 & & & & \\
\hline
\end{tabular}


J. R. Walsh et al.: Abundance distribution in NGC 5128 from PNe

Table 11. continued.

\begin{tabular}{|c|c|c|c|c|c|c|c|c|c|c|c|}
\hline $\mathrm{H} \alpha$ & 6562 & 367 & 25 & 462 & 46 & 437 & 29 & 361 & 18 & 349 & 30 \\
\hline$[\mathrm{N} \mathrm{II}]$ & 6583 & 78 & 7 & 231 & 26 & 203 & 15 & 147 & 8 & 18 & 7 \\
\hline [S II] & 6716 & 20 & 5 & & & & & 6 & 2 & & \\
\hline [S II] & 6730 & 27 & 7 & & & & & 12 & 4 & 26 & 11 \\
\hline [Ar III] & 7133 & 23 & 6 & & & 37 & 8 & 30 & 5 & & \\
\hline [O II] & 7325 & & & & & & & & & 37 & 6 \\
\hline $\log F(\mathrm{H} \beta)$ & & -16.56 & 0.03 & -16.78 & 0.04 & -16.62 & 0.03 & -16.43 & 0.02 & -16.62 & 0.04 \\
\hline \multirow{2}{*}{$m_{5007 \mathrm{~A}}$} & & 25.09 & 0.07 & 25.04 & 0.10 & 25.89 & 0.07 & 25.27 & 0.05 & 25.59 & 0.09 \\
\hline & & \multicolumn{2}{|c|}{$\begin{array}{c}\text { F42 \#7 } \\
0519 \\
(-253.4,-81.8) \\
\end{array}$} & \multicolumn{2}{|c|}{$\begin{array}{c}\text { F42 \#8 } \\
4217 \\
(-229.7,-119.5) \\
\end{array}$} & \multicolumn{2}{|c|}{$\begin{array}{c}\text { F42 \#9 } \\
4244 \\
(-209.0,-72.3) \\
\end{array}$} & \multicolumn{2}{|c|}{$\begin{array}{c}\text { F42 \#10 } \\
4211 \\
(-179.6,-119.8) \\
\end{array}$} & \multicolumn{2}{|c|}{$\begin{array}{c}\mathrm{F} 42 \# 11 \\
0509 \\
(-165.4,-52.4) \\
\end{array}$} \\
\hline Species & $\lambda(\AA)$ & $F_{\mathrm{Obs}}$ & \pm & $F_{\mathrm{Obs}}$ & \pm & $F_{\mathrm{Obs}}$ & \pm & $F_{\mathrm{Obs}}$ & \pm & $F_{\mathrm{Obs}}$ & \pm \\
\hline$[\mathrm{O} \mathrm{II}]$ & 3727 & & & 40 & 7 & 12 & 8 & 48 & 4 & & \\
\hline$[\mathrm{Ne} \mathrm{III}]$ & 3868 & 80 & 9 & 78 & 8 & 66 & 10 & 54 & 5 & 45 & 7 \\
\hline$[\mathrm{Ne} \mathrm{III}]+\mathrm{H} \epsilon$ & 3970 & 36 & 7 & 31 & 7 & 27 & 10 & 18 & 3 & & \\
\hline $\mathrm{H} \delta$ & 4101 & 20 & 6 & 18 & 6 & & & 22 & 5 & 19 & 5 \\
\hline $\mathrm{H} \gamma$ & 4340 & 40 & 7 & 39 & 7 & 50 & 33 & 44 & 7 & 43 & 10 \\
\hline [O III] & 4363 & & & & & & & 10 & 6 & & \\
\hline He I & 4471 & & & & & & & & & & \\
\hline He II & 4686 & 23 & 8 & & & & & 20 & 4 & & \\
\hline $\mathrm{H} \beta$ & 4861 & 100 & 0 & 100 & 0 & 100 & 0 & 100 & 0 & 100 & 0 \\
\hline [O III] & 4959 & 512 & 42 & 431 & 31 & 339 & 30 & 271 & 14 & 313 & 26 \\
\hline [O III] & 5007 & 1522 & 121 & 1315 & 90 & 1081 & 90 & 827 & 41 & 973 & 78 \\
\hline $\mathrm{He} \mathrm{I}$ & 5876 & & & & & 29 & 12 & & & & \\
\hline$[\mathrm{N} \mathrm{II}]$ & 6548 & & & & & & & 58 & 20 & 117 & 24 \\
\hline $\mathrm{H} \alpha$ & 6562 & 410 & 34 & 390 & 28 & 412 & 37 & 447 & 23 & 372 & 33 \\
\hline [N II] & 6583 & 142 & 16 & 161 & 14 & 118 & 14 & 278 & 15 & 226 & 21 \\
\hline $\mathrm{He} \mathrm{I}$ & 6678 & & & & & & & 15 & 4 & & \\
\hline [S II] & 6716 & & & & & & & 22 & 5 & & \\
\hline [S II] & 6730 & & & & & & & 37 & 7 & 16 & 7 \\
\hline [Ar III] & 7133 & & & & & 48 & 10 & 29 & 6 & 84 & 25 \\
\hline$[\mathrm{O} \mathrm{II}]$ & 7325 & & & & & 72 & 31 & & & & \\
\hline $\log F(\mathrm{H} \beta)$ & & -16.63 & 0.03 & -16.60 & 0.03 & -16.70 & 0.04 & -16.38 & 0.02 & -16.59 & 0.04 \\
\hline \multirow[t]{2}{*}{$m_{5007 \mathrm{~A}}$} & & 24.87 & 0.09 & 24.97 & 0.08 & 25.43 & 0.09 & 24.90 & 0.05 & 25.25 & 0.09 \\
\hline & & \multicolumn{2}{|c|}{$\begin{array}{c}\mathrm{F} 42 \# 12 \mathrm{~b} \\
4214 \\
(-135.0,-115.2) \\
\end{array}$} & \multicolumn{2}{|c|}{$\begin{array}{c}\mathrm{F} 42 \# 13 \\
4248 \\
(-115.2,-103.1) \\
\end{array}$} & \multicolumn{2}{|c|}{$\begin{array}{c}\mathrm{F} 42 \# 14 \mathrm{~b} \\
4210 \\
(-99.8,-73.0) \\
\end{array}$} & \multicolumn{2}{|c|}{$\begin{array}{c}\mathrm{F} 42 \# 15 \mathrm{~b} \\
4245 \\
(-68.7,-65.2) \\
\end{array}$} & \multicolumn{2}{|c|}{$\begin{array}{c}\text { F42 \#16a } \\
4207 \\
(-48.4,-102.4) \\
\end{array}$} \\
\hline Species & $\lambda(\AA)$ & $F_{\text {Obs }}$ & \pm & $F_{\mathrm{Obs}}$ & \pm & $F_{\text {Obs }}$ & \pm & $F_{\text {Obs }}$ & \pm & $F_{\text {Obs }}$ & \pm \\
\hline [O II] & 3727 & & & 56 & 32 & 50 & 38 & & & 65 & 14 \\
\hline$[\mathrm{Ne} \mathrm{III}]$ & 3868 & 114 & 15 & 151 & 80 & 160 & 32 & 43 & 9 & 100 & 16 \\
\hline$[\mathrm{Ne} \mathrm{III}]+\mathrm{H} \epsilon$ & 3970 & 64 & 10 & 53 & 32 & & & & & 34 & 10 \\
\hline $\mathrm{H} \delta$ & 4101 & & & & & & & & & & \\
\hline $\mathrm{H} \gamma$ & 4340 & 40 & 12 & & & & & 31 & 17 & 44 & 15 \\
\hline [O III] & 4363 & & & & & & & & & 19 & 8 \\
\hline $\mathrm{He} \mathrm{I}$ & 4471 & & & & & & & & & & \\
\hline He II & 4686 & 14 & 12 & 31 & 24 & 58 & 29 & & & & \\
\hline $\mathrm{H} \beta$ & 4861 & 100 & 0 & 100 & 0 & 100 & 0 & 100 & 0 & 100 & 0 \\
\hline [O III] & 4959 & 569 & 57 & 690 & 356 & 796 & 140 & 270 & 33 & 574 & 74 \\
\hline [O III] & 5007 & 1747 & 172 & 2185 & 1125 & 2438 & 426 & 872 & 101 & 1749 & 224 \\
\hline He I & 5876 & & & & & & & & & & \\
\hline$[\mathrm{N} \mathrm{II}]$ & 6548 & & & & & & & & & 64 & 16 \\
\hline $\mathrm{H} \alpha$ & 6562 & 504 & 51 & 507 & 263 & 493 & 89 & 432 & 51 & 385 & 52 \\
\hline [N II] & 6583 & 197 & 23 & 246 & 130 & 171 & 36 & & & 192 & 30 \\
\hline $\mathrm{He} \mathrm{I}$ & 6678 & & & & & & & & & & \\
\hline [S II $]$ & 6716 & & & & & & & & & & \\
\hline [S II] & 6730 & & & & & & & 35 & 10 & & \\
\hline [Ar III] & 7133 & & & & & & & & & & \\
\hline$[\mathrm{O} \mathrm{II}]$ & 7325 & & & & & & & & & 44 & 19 \\
\hline $\log F(\mathrm{H} \beta)$ & & -16.73 & 0.04 & -17.10 & 0.22 & -16.88 & 0.08 & -16.64 & 0.05 & -16.65 & 0.06 \\
\hline$m_{5007 \mathrm{~A}}$ & & 24.97 & 0.11 & 25.66 & 0.56 & 24.99 & 0.19 & 25.51 & 0.13 & 24.77 & 0.14 \\
\hline
\end{tabular}


Table 11. continued.

\begin{tabular}{|c|c|c|c|c|c|c|c|c|c|c|c|}
\hline \multirow[b]{2}{*}{ Species } & \multirow[b]{2}{*}{$\lambda(\AA)$} & \multicolumn{2}{|c|}{$\begin{array}{c}4264 \\
(-13.9,-166.9) \\
\end{array}$} & \multicolumn{2}{|c|}{$\begin{array}{c}4212 \\
(-5.9,-115.2) \\
\end{array}$} & & & & & & \\
\hline & & $F_{\mathrm{Obs}}$ & \pm & $F_{\mathrm{Obs}}$ & \pm & & & & & & \\
\hline$[\mathrm{O} \mathrm{II}]$ & 3727 & \multirow{8}{*}{34} & \multirow{7}{*}{8} & \multirow{4}{*}{40} & \multirow{3}{*}{9} & & & & & & \\
\hline [Ne III] & 3868 & & & & & & & & & & \\
\hline$[\mathrm{Ne} \mathrm{III}]+\mathrm{H} \epsilon$ & 3970 & & & & & & & & & & \\
\hline $\mathrm{H} \delta$ & 4101 & & & & \multirow{3}{*}{11} & & & & & & \\
\hline $\mathrm{H} \gamma$ & 4340 & & & \multirow{3}{*}{46} & & & & & & & \\
\hline [O III] & 4363 & & & & & & & & & & \\
\hline $\mathrm{He} \mathrm{I}$ & 4471 & & & & & & & & & & \\
\hline $\mathrm{He}$ II & 4686 & & & 17 & 14 & & & & & & \\
\hline $\mathrm{H} \beta$ & 4861 & 100 & 0 & 100 & 0 & & & & & & \\
\hline [O III] & 4959 & 210 & 16 & 553 & 61 & & & & & & \\
\hline [O III] & 5007 & 608 & 42 & 1616 & 175 & & & & & & \\
\hline He I & 5876 & & & & & & & & & & \\
\hline$[\mathrm{N} \mathrm{II}]$ & 6548 & 51 & 11 & & & & & & & & \\
\hline $\mathrm{H} \alpha$ & 6562 & 495 & 36 & 583 & 65 & & & & & & \\
\hline$[\mathrm{N} \mathrm{II}]$ & 6583 & 191 & 18 & 69 & 16 & & & & & & \\
\hline $\mathrm{He} \mathrm{I}$ & 6678 & & & & & & & & & & \\
\hline [S II $]$ & 6716 & & & & & & & & & & \\
\hline [S II] & 6730 & & & & & & & & & & \\
\hline [Ar III] & 7133 & & & & & & & & & & \\
\hline [O II] & 7325 & & & & & & & & & & \\
\hline $\log F(\mathrm{H} \beta)$ & & -16.61 & 0.03 & -16.65 & 0.05 & & & & & & \\
\hline \multirow{2}{*}{$m_{5007 \mathrm{~A}}$} & & 25.81 & 0.07 & 24.85 & 0.12 & & & & & & \\
\hline & & \multicolumn{2}{|c|}{$\begin{array}{c}\text { F34 \#1 } \\
2606 \\
(-497.0,-921.7) \\
\end{array}$} & \multicolumn{2}{|c|}{$\begin{array}{c}\text { F34 \#2 } \\
2603 \\
(-548.8,-902.6)\end{array}$} & \multicolumn{2}{|c|}{$\begin{array}{c}\text { F34 \#4 } \\
2607 \\
-446.9,-860.0) \\
\end{array}$} & $\begin{array}{r}\mathrm{F} 3 \\
3 \\
(-433.1 \\
\end{array}$ & $\begin{array}{l}7 \\
790.8) \\
\end{array}$ & $\begin{array}{r}\mathrm{F} 3 \\
3 \\
(-409 . \\
\end{array}$ & $\begin{array}{l}11 \\
693.3) \\
\end{array}$ \\
\hline Species & $\lambda(\AA)$ & $F_{\mathrm{Obs}}$ & \pm & $F_{\mathrm{Obs}}$ & \pm & $F_{\text {Obs }}$ & \pm & $F_{\text {Obs }}$ & \pm & $F_{\text {Obs }}$ & \pm \\
\hline$[\mathrm{O} \mathrm{II}]$ & 3727 & 64 & 7 & 64 & 7 & 48 & 9 & 55 & 28 & 30 & 11 \\
\hline$[\mathrm{Ne} \mathrm{III}]$ & 3868 & 72 & 5 & 45 & 5 & 49 & 5 & 98 & 6 & 95 & 11 \\
\hline$[\mathrm{Ne} \mathrm{III}]+\mathrm{H} \epsilon$ & 3970 & 25 & 4 & & & 35 & 6 & 39 & 5 & 28 & 7 \\
\hline $\mathrm{H} \delta$ & 4101 & 23 & 4 & 16 & 4 & 24 & 4 & 25 & 5 & 24 & 6 \\
\hline $\mathrm{H} \gamma$ & 4340 & 35 & 3 & 44 & 5 & 46 & 5 & 43 & 6 & 49 & 8 \\
\hline [O III] & 4363 & & & 9 & 4 & & & 8 & 2 & 18 & 9 \\
\hline He I & 4471 & & & 17 & 4 & & & 9 & 3 & 18 & 7 \\
\hline He II & 4686 & 2 & 2 & 8 & 4 & & & $\mathrm{t}$ & & 48 & 9 \\
\hline $\mathrm{H} \beta$ & 4861 & 100 & 0 & 100 & 0 & 100 & 0 & 100 & 0 & 100 & 0 \\
\hline [O III] & 4959 & 404 & 15 & 261 & 13 & 319 & 16 & 535 & 21 & 530 & 45 \\
\hline [O III] & 5007 & 1214 & 43 & 782 & 37 & 972 & 45 & 1591 & 62 & 1574 & 130 \\
\hline $\mathrm{He} \mathrm{I}$ & 5876 & & & 32 & 9 & & & & & & \\
\hline$[\mathrm{N} \mathrm{III]}$ & 6548 & 33 & 9 & & & & & & & & \\
\hline $\mathrm{H} \alpha$ & 6562 & 410 & 17 & 528 & 28 & 454 & 24 & 447 & 20 & 421 & 43 \\
\hline$[\mathrm{N} \mathrm{II]}$ & 6583 & 152 & 9 & 108 & 12 & 151 & 11 & 116 & 9 & 27 & 11 \\
\hline $\mathrm{He} \mathrm{I}$ & 6678 & & & 26 & 6 & & & & & 8 & 8 \\
\hline [S II] & 6716 & & & & & & & & & & \\
\hline [S II] & 6730 & & & & & & & & & & \\
\hline [Ar III] & 7133 & 6 & 4 & & & 39 & 10 & 37 & 7 & & \\
\hline [O II $]$ & 7325 & 6 & 5 & & & 33 & 16 & & & & \\
\hline $\log F(\mathrm{H} \beta)$ & & -16.56 & 0.02 & -16.70 & 0.03 & -16.69 & 0.03 & -16.59 & 0.03 & -16.94 & 0.05 \\
\hline$m_{5007 \mathrm{~A}}$ & & 24.96 & 0.04 & 25.78 & 0.05 & 25.51 & 0.05 & 24.73 & 0.04 & 25.62 & 0.09 \\
\hline & & $\begin{array}{r}\mathrm{F} 3 \\
3 \\
(-424.4 \\
\end{array}$ & $\begin{array}{l}12 \\
5 \\
682.1) \\
\end{array}$ & $\begin{array}{r}\mathrm{F} 3 \\
3 \\
(-458, \\
\end{array}$ & $\begin{array}{l}14 \\
5 \\
-622.8) \\
\end{array}$ & $\begin{array}{r}\mathrm{F} 3 \\
3 \\
(-490.7 \\
\end{array}$ & $\begin{array}{l}15 \\
611.0) \\
\end{array}$ & $\begin{array}{r}\mathrm{F} 3 \\
3 \\
(-434.5 \\
\end{array}$ & $\begin{array}{l}16 \\
592.1) \\
\end{array}$ & & \\
\hline Species & $\lambda(\AA)$ & $F_{\mathrm{Obs}}$ & \pm & $F_{\mathrm{Obs}}$ & \pm & $F_{\text {Obs }}$ & \pm & $F_{\text {Obs }}$ & \pm & & \\
\hline [O II] & 3727 & 48 & 16 & 38 & 4 & & & 138 & 16 & & \\
\hline$[\mathrm{Ne} \mathrm{III}]$ & 3868 & 34 & 7 & 66 & 5 & 93 & 9 & 111 & 14 & & \\
\hline$[\mathrm{Ne} \mathrm{III}]+\mathrm{H} \epsilon$ & 3970 & & & 30 & 4 & & & 46 & 9 & & \\
\hline $\mathrm{H} \delta$ & 4101 & & & 15 & 10 & 20 & 6 & & & & \\
\hline $\mathrm{H} \gamma$ & 4340 & 35 & 8 & 47 & 5 & 45 & 6 & 46 & 12 & & \\
\hline [O III] & 4363 & & & 7 & 3 & 13 & 5 & 25 & 8 & & \\
\hline
\end{tabular}


J. R. Walsh et al.: Abundance distribution in NGC 5128 from PNe

Table 11. continued.

\begin{tabular}{ll|cc|cc|cc|cc|c}
\hline \hline $\mathrm{He}$ I & 4471 & & & 5 & 3 & & & & & \\
$\mathrm{He}$ II & 4686 & 45 & 9 & & & 21 & 15 & 47 & 12 & \\
$\mathrm{H} \beta$ & 4861 & 100 & 0 & 100 & 0 & 100 & 0 & 100 & 0 & \\
{$[\mathrm{O} \mathrm{III}]$} & 4959 & 477 & 45 & 403 & 18 & 455 & 30 & 532 & 50 & \\
{$[\mathrm{O} \mathrm{III}]$} & 5007 & 1426 & 132 & 1193 & 53 & 1349 & 87 & 1647 & 151 & \\
$\mathrm{He} \mathrm{I}$ & 5876 & 49 & 25 & & & & & & & \\
{$[\mathrm{~N} \mathrm{II}]$} & 6548 & & & & & & & 88 & 13 & \\
$\mathrm{H} \alpha$ & 6562 & 321 & 30 & 380 & 19 & 475 & 34 & 458 & 48 & \\
{$[\mathrm{~N} \mathrm{III}]$} & 6583 & 21 & 8 & 59 & 7 & 43 & 11 & 418 & 44 & \\
$\mathrm{He} \mathrm{I}$ & 6678 & & & & & & & & & \\
{$[\mathrm{~S} \mathrm{II}]$} & 6716 & & & & & & & & & \\
{$[\mathrm{~S} \mathrm{II}]$} & 6730 & & & & & & & & & \\
{$[\mathrm{Ar} \mathrm{III}]$} & 7133 & & & & & & & & & \\
{$[\mathrm{O} \mathrm{II}]$} & 7325 & 57 & 26 & & & & & & & \\
\hline $\log F(\mathrm{H} \beta)$ & & -16.98 & 0.05 & -16.63 & 0.03 & -16.83 & 0.04 & -17.03 & 0.04 & \\
$m_{5007 \mathrm{~A}}$ & & 25.84 & 0.10 & 25.16 & 0.05 & 25.50 & 0.07 & 25.81 & 0.10 & \\
\hline
\end{tabular}


Table 12. NGC 5128 PN spectra - extinction corrected line fluxes.

\begin{tabular}{|c|c|c|c|c|c|c|c|c|c|c|c|}
\hline \multirow[b]{2}{*}{ Species } & \multirow[b]{2}{*}{$\lambda(\AA)$} & \multicolumn{2}{|c|}{$\begin{array}{c}\text { F56\#1 } \\
5615 \\
(+297.6,-492.5)\end{array}$} & \multicolumn{2}{|c|}{$\begin{array}{c}\text { F56 \#2 } \\
5601 \\
(+284.3,-465.9) \\
\end{array}$} & \multicolumn{2}{|c|}{$\begin{array}{c}\text { F56 \#3 } \\
5621 \\
(+287.4,-448.7) \\
\end{array}$} & \multicolumn{2}{|c|}{$\begin{array}{c}\text { F56 \#4 } \\
5611 \\
(+298.7,-405.0) \\
\end{array}$} & \multicolumn{2}{|c|}{$\begin{array}{c}\text { F56 \#5 } \\
5608 \\
(+268.9,-394.1) \\
\end{array}$} \\
\hline & & $F_{\text {Dered }}$ & \pm & $F_{\text {Dered }}$ & \pm & $F_{\text {Dered }}$ & \pm & $F_{\text {Dered }}$ & \pm & $F_{\text {Dered }}$ & \pm \\
\hline [O II] & 3727 & 50 & 42 & 56 & 13 & 120 & 29 & & & & \\
\hline$[\mathrm{Ne}$ III] $]$ & 3868 & 49 & 18 & 124 & 13 & 76 & 22 & 95 & 25 & 150 & 81 \\
\hline$[\mathrm{Ne} \mathrm{III}]+\mathrm{H} \epsilon$ & 3970 & & & 37 & 8 & & & & & 72 & 42 \\
\hline $\mathrm{H} \delta$ & 4101 & & & 27 & 10 & & & & & & \\
\hline $\mathrm{H} \gamma$ & 4340 & 41 & 15 & 58 & 10 & 51 & 17 & 35 & 14 & & \\
\hline [O III] & 4363 & & & 28 & 8 & & & & & & \\
\hline $\mathrm{He} \mathrm{I}$ & 4471 & & & 6 & 3 & & & & & & \\
\hline $\mathrm{He}$ II & 4686 & & & 39 & 8 & & & & & & \\
\hline $\mathrm{H} \beta$ & 4861 & 100 & 0 & 100 & 0 & 100 & 0 & 100 & 0 & 100 & 0 \\
\hline [O III] & 4959 & 291 & 26 & 457 & 31 & 268 & 34 & 323 & 47 & 533 & 62 \\
\hline [O III] & 5007 & 836 & 66 & 1367 & 90 & 772 & 87 & 925 & 129 & 1536 & 166 \\
\hline He I & 5876 & & & 6 & 3 & & & 18 & 5 & & \\
\hline$[\mathrm{N} \mathrm{II}]$ & 6548 & 31 & 5 & 18 & 4 & 46 & 10 & 51 & 10 & & \\
\hline $\mathrm{H} \alpha$ & 6562 & 282 & 23 & 281 & 19 & 282 & 32 & 282 & 40 & 281 & 32 \\
\hline$[\mathrm{N} \mathrm{II}]$ & 6583 & 105 & 10 & 55 & 5 & 181 & 21 & 168 & 24 & 17 & 7 \\
\hline $\mathrm{He} \mathrm{I}$ & 6678 & & & 7 & 4 & & & & & & \\
\hline [S II $]$ & 6716 & & & & & 24 & 19 & 14 & 5 & & \\
\hline [S II] & 6730 & 9 & 5 & & & 13 & 7 & 7 & 4 & & \\
\hline [Ar III] & 7133 & 11 & 6 & 8 & 3 & 12 & 6 & 37 & 8 & 24 & 21 \\
\hline$[\mathrm{O} \mathrm{II}]$ & 7325 & & & 14 & 4 & 58 & 20 & 17 & 7 & & \\
\hline $\mathrm{c}$ & & 0.46 & 0.11 & 0.48 & 0.09 & 0.62 & 0.16 & 0.53 & 0.20 & 0.56 & 0.16 \\
\hline $\log F(\mathrm{H} \beta)$ & & -15.91 & 0.03 & -15.58 & 0.03 & -15.88 & 0.05 & -15.86 & 0.06 & -16.03 & 0.05 \\
\hline \multirow[t]{2}{*}{$m_{5007 \mathrm{~A}}$} & & 23.73 & 0.09 & 22.37 & 0.07 & 23.73 & 0.12 & 23.49 & 0.15 & 23.37 & 0.12 \\
\hline & & \multicolumn{2}{|c|}{$\begin{array}{c}\text { F56 \#6 } \\
5602 \\
47.7,-372.5) \\
\end{array}$} & \multicolumn{2}{|c|}{$\begin{array}{c}\text { F56 \#8 } \\
5425 \\
(+260.2,-326.0) \\
\end{array}$} & \multicolumn{2}{|c|}{$\begin{array}{c}\text { F56 \#9 } \\
5456 \\
(+235.0,-312.2) \\
\end{array}$} & \multicolumn{2}{|c|}{$\begin{array}{c}\text { F56 \#10 } \\
5416 \\
(+241.5,-281.6) \\
\end{array}$} & \multicolumn{2}{|c|}{$\begin{array}{c}\text { F56\#11 } \\
5409 \\
(+240.2,-279.3) \\
\end{array}$} \\
\hline Species & $\lambda(\AA)$ & $F_{\text {Dered }}$ & \pm & $F_{\text {Dered }}$ & \pm & $F_{\text {Dered }}$ & \pm & $F_{\text {Dered }}$ & \pm & $F_{\text {Dered }}$ & \pm \\
\hline$[\mathrm{O} \mathrm{II}]$ & 3727 & 63 & 13 & 46 & 12 & & & 31 & 19 & 33 & 6 \\
\hline [Ne III] & 3868 & 109 & 17 & 59 & 15 & & & 87 & 14 & 109 & 11 \\
\hline$[\mathrm{Ne} \mathrm{III}]+\mathrm{H} \epsilon$ & 3970 & 73 & 11 & 36 & 19 & & & 66 & 38 & 52 & 26 \\
\hline $\mathrm{H} \delta$ & 4101 & 18 & 13 & 32 & 20 & & & 33 & 9 & 30 & 11 \\
\hline $\mathrm{H} \gamma$ & 4340 & 53 & 8 & 47 & 11 & & & 49 & 10 & 51 & 10 \\
\hline [O III] & 4363 & & & & & & & & & & \\
\hline $\mathrm{He} \mathrm{I}$ & 4471 & & & & & & & & & & \\
\hline $\mathrm{He}$ II & 4686 & & & & & & & & & & \\
\hline $\mathrm{H} \beta$ & 4861 & 100 & 0 & 100 & 0 & 100 & 0 & 100 & 0 & 100 & 0 \\
\hline [O III] & 4959 & 416 & 34 & 286 & 27 & 262 & 107 & 373 & 29 & 470 & 36 \\
\hline [O III] & 5007 & 1203 & 94 & 841 & 76 & 789 & 309 & 1137 & 84 & 1344 & 101 \\
\hline $\mathrm{He} \mathrm{I}$ & 5876 & 10 & 2 & 28 & 7 & & & 21 & 6 & 10 & 6 \\
\hline$[\mathrm{N} \mathrm{II}]$ & 6548 & 23 & 3 & 59 & 7 & & & 21 & 4 & 49 & 10 \\
\hline $\mathrm{H} \alpha$ & 6562 & 281 & 22 & 282 & 26 & 282 & 115 & 281 & 21 & 282 & 64 \\
\hline [N II] & 6583 & 89 & 8 & 181 & 17 & 48 & 34 & 81 & 7 & 135 & 29 \\
\hline $\mathrm{He} \mathrm{I}$ & 6678 & 10 & 3 & 14 & 9 & & & & & & \\
\hline [S II] & 6716 & & & 10 & 3 & & & & & & \\
\hline [S II] & 6730 & & & 14 & 4 & & & 10 & 5 & 9 & 2 \\
\hline [Ar III] & 7133 & 30 & 5 & 29 & 6 & & & 34 & 5 & 25 & 6 \\
\hline [O II $]$ & 7325 & 19 & 8 & 33 & 11 & & & 16 & 12 & & \\
\hline $\mathrm{c}$ & & 0.71 & 0.11 & 0.35 & 0.13 & 0.09 & 0.30 & 0.45 & 0.11 & 0.24 & 0.32 \\
\hline $\log F(\mathrm{H} \beta)$ & & -15.37 & 0.03 & -15.78 & 0.04 & -17.37 & 0.19 & -15.69 & 0.03 & -15.88 & 0.03 \\
\hline \multirow[t]{2}{*}{$m_{5007 \mathrm{~A}}$} & & 21.99 & 0.09 & 23.39 & 0.10 & 27.44 & 0.43 & 23.93 & 0.08 & 23.13 & 0.08 \\
\hline & & \multicolumn{2}{|c|}{$\begin{array}{c}\text { F56 \#12a } \\
5418 \\
(+221.9,-245.1) \\
\end{array}$} & \multicolumn{2}{|c|}{$\begin{array}{c}\text { F56 \#12b } \\
5438 \\
(+220.2,-242.0) \\
\end{array}$} & $\begin{array}{r}\mathrm{F} 56 \\
5 \\
(+201.5 \\
\end{array}$ & $\begin{array}{l}3 \mathrm{~b} \\
225.7)\end{array}$ & $\begin{array}{r}\mathrm{F} 56 \\
5 \\
(+183.5 \\
\end{array}$ & $\begin{array}{l}14 \\
8 \\
-217.4) \\
\end{array}$ & $\begin{array}{r}\mathrm{F} 5 \\
5 \\
(+169.1 \\
\end{array}$ & $\begin{array}{l}15 \\
1 \\
-200.2) \\
\end{array}$ \\
\hline Species & $\lambda(\AA)$ & $F_{\text {Dered }}$ & \pm & $F_{\text {Dered }}$ & \pm & $F_{\text {Dered }}$ & \pm & $F_{\text {Dered }}$ & \pm & $F_{\text {Dered }}$ & \pm \\
\hline$[\mathrm{O} I \mathrm{II}]$ & 3727 & 106 & 72 & 19 & 14 & 40 & 16 & 36 & 14 & & \\
\hline$[\mathrm{Ne}$ III $]$ & 3868 & 172 & 35 & 39 & 13 & 102 & 17 & 105 & 14 & 73 & 16 \\
\hline
\end{tabular}


J. R. Walsh et al.: Abundance distribution in NGC 5128 from PNe

Table 12. continued.

\begin{tabular}{|c|c|c|c|c|c|c|c|c|c|c|c|}
\hline$[\mathrm{Ne} \mathrm{III}]+\mathrm{H} \epsilon$ & 3970 & \multirow[t]{2}{*}{85} & \multirow[t]{2}{*}{$\overline{633}$} & 32 & 16 & \multirow[t]{2}{*}{48} & \multirow[t]{2}{*}{15} & \multirow{2}{*}{$\begin{array}{l}61 \\
30\end{array}$} & 49 & \multirow[b]{2}{*}{32} & \multirow[b]{2}{*}{16} \\
\hline $\mathrm{H} \delta$ & 4101 & & & 18 & 9 & & & & 11 & & \\
\hline $\mathrm{H} \gamma$ & 4340 & \multirow[t]{3}{*}{48} & \multirow[t]{3}{*}{15} & \multirow[t]{4}{*}{40} & \multirow[t]{4}{*}{10} & \multirow[t]{3}{*}{46} & \multirow[t]{3}{*}{16} & \multirow[t]{4}{*}{35} & 11 & 52 & 15 \\
\hline [O III] & 4363 & & & & & & & & & & \\
\hline He I & 4471 & & & & & & & & & & \\
\hline He II & 4686 & 60 & 14 & & & 14 & 11 & & & & \\
\hline $\mathrm{H} \beta$ & 4861 & 100 & 0 & 100 & 0 & 100 & 0 & 100 & 0 & 100 & 0 \\
\hline [O III] & 4959 & 665 & 94 & 177 & 19 & 517 & 48 & 435 & 40 & 356 & 44 \\
\hline [O III] & 5007 & 1872 & 259 & 502 & 49 & 1488 & 136 & 1292 & 117 & 1037 & 123 \\
\hline $\mathrm{He} \mathrm{I}$ & 5876 & 15 & 9 & 14 & 7 & 12 & 5 & 19 & 5 & & \\
\hline$[\mathrm{N} \mathrm{II}]$ & 6548 & 75 & 13 & 60 & 7 & & & 50 & 7 & 17 & 6 \\
\hline $\mathrm{H} \alpha$ & 6562 & 283 & 40 & 282 & 28 & 281 & 26 & 283 & 26 & 282 & 34 \\
\hline$[\mathrm{N} \mathrm{II}]$ & 6583 & 225 & 32 & 205 & 20 & 25 & 5 & 143 & 14 & 42 & 7 \\
\hline $\mathrm{He} \mathrm{I}$ & 6678 & & & & & 8 & 3 & & & & \\
\hline [S II $]$ & 6716 & & & & & & & & & & \\
\hline [S II $]$ & 6730 & 8 & 6 & & & & & 8 & 4 & 13 & 6 \\
\hline [Ar III] & 7133 & 19 & 5 & 23 & 5 & 13 & 6 & 23 & 5 & 13 & 5 \\
\hline [O II] & 7325 & 42 & 13 & 13 & 8 & & & 37 & 10 & & \\
\hline $\mathrm{c}$ & & 0.55 & 0.20 & 0.36 & 0.14 & 0.43 & 0.13 & 0.42 & 0.13 & 0.54 & 0.17 \\
\hline $\log F(\mathrm{H} \beta)$ & & -15.83 & 0.06 & -15.81 & 0.04 & -15.79 & 0.04 & -15.69 & 0.04 & -15.67 & 0.05 \\
\hline$m_{5007 \mathrm{~A}}$ & & 22.64 & 0.15 & 24.04 & 0.11 & 22.79 & 0.10 & 22.70 & 0.10 & 24.19 & 0.13 \\
\hline & & $\begin{array}{r}\text { F5 } \\
5 \\
(+139.3 \\
\end{array}$ & $\begin{array}{l}16 \\
9 \\
-175.8) \\
\end{array}$ & $\begin{array}{r}\mathrm{F} 5 \\
5 \\
(+146.7 \\
\end{array}$ & $\begin{array}{l}17 \\
145.7) \\
\end{array}$ & $\begin{array}{r}\mathrm{F} 5 \\
5 \\
(+107.6 \\
\end{array}$ & $\begin{array}{l}18 \\
151.4) \\
\end{array}$ & & & & \\
\hline Species & $\lambda(\AA)$ & $F_{\text {Dered }}$ & \pm & $F_{\text {Dered }}$ & \pm & $F_{\text {Dered }}$ & \pm & & & & \\
\hline [O II] & 3727 & 57 & 21 & & & 37 & 26 & & & & \\
\hline [Ne III] & 3868 & 63 & 16 & 31 & 12 & 123 & 30 & & & & \\
\hline$[\mathrm{Ne} \mathrm{III}]+\mathrm{H} \epsilon$ & 3970 & 21 & 9 & 21 & 12 & 40 & 20 & & & & \\
\hline $\mathrm{H} \delta$ & 4101 & & & & & & & & & & \\
\hline $\mathrm{H} \gamma$ & 4340 & 43 & 9 & 34 & 9 & & & & & & \\
\hline [O III] & 4363 & & & & & & & & & & \\
\hline $\mathrm{He} \mathrm{I}$ & 4471 & 22 & 8 & & & & & & & & \\
\hline $\mathrm{He}$ II & 4686 & & & & & & & & & & \\
\hline $\mathrm{H} \beta$ & 4861 & 100 & 0 & 100 & 0 & 100 & 0 & & & & \\
\hline [O III] & 4959 & 359 & 30 & 270 & 33 & 402 & 72 & & & & \\
\hline [O III] & 5007 & 1018 & 81 & 840 & 98 & 1249 & 218 & & & & \\
\hline $\mathrm{He} \mathrm{I}$ & 5876 & 19 & 7 & 26 & 11 & 20 & 10 & & & & \\
\hline$[\mathrm{N} \mathrm{II}]$ & 6548 & 25 & 5 & & & 64 & 15 & & & & \\
\hline $\mathrm{H} \alpha$ & 6562 & 282 & 23 & 283 & 33 & 282 & 50 & & & & \\
\hline$[\mathrm{N} \mathrm{II}]$ & 6583 & 100 & 10 & & & 203 & 36 & & & & \\
\hline $\mathrm{He} \mathrm{I}$ & 6678 & & & & & & & & & & \\
\hline [S II $]$ & 6716 & & & & & & & & & & \\
\hline [S II] & 6730 & 7 & 4 & & & 18 & 7 & & & & \\
\hline [Ar III] & 7133 & 15 & 5 & & & & & & & & \\
\hline$[\mathrm{O} \mathrm{II}]$ & 7325 & & & & & & & & & & \\
\hline $\mathrm{c}$ & & 0.54 & 0.11 & 0.32 & 0.17 & 0.64 & 0.25 & & & & \\
\hline $\log F(\mathrm{H} \beta)$ & & -15.61 & 0.03 & -15.87 & 0.05 & -15.71 & 0.08 & & & & \\
\hline$m_{5007 \mathrm{~A}}$ & & 22.77 & 0.09 & 23.63 & 0.13 & 22.90 & 0.19 & & & & \\
\hline & & $\begin{array}{r}\mathrm{F} 4 \\
4 \\
(-393 . \\
\end{array}$ & $\begin{array}{l}1 \\
4 \\
-84.9) \\
\end{array}$ & $\begin{array}{r}\mathrm{F} 4 \\
4 \\
(-362 . \\
\end{array}$ & $\begin{array}{l}2 \\
-71.3) \\
\end{array}$ & $\begin{array}{r}\mathrm{F} 4 \\
4 \\
(-345.6 \\
\end{array}$ & $\begin{array}{l}3 \\
126.0) \\
\end{array}$ & $\begin{array}{r}\mathrm{F} 4 \\
4 \\
(-318 \\
\end{array}$ & $\begin{array}{l}4 \\
6 \\
-79.3) \\
\end{array}$ & $\begin{array}{r}\mathrm{F} \\
0 \\
(-278 \\
\end{array}$ & $\begin{array}{l}6 \\
-34.0) \\
\end{array}$ \\
\hline Species & $\lambda(\AA)$ & $F_{\text {Dered }}$ & \pm & $F_{\text {Dered }}$ & \pm & $F_{\text {Dered }}$ & \pm & $F_{\text {Dered }}$ & \pm & $F_{\text {Dered }}$ & \pm \\
\hline [O II] & 3727 & & & 111 & 56 & & & 26 & 7 & 52 & 40 \\
\hline [Ne III] & 3868 & 74 & 10 & 192 & 24 & 26 & 5 & 34 & 5 & 50 & 9 \\
\hline$[\mathrm{Ne} \mathrm{III}]+\mathrm{H} \epsilon$ & 3970 & 40 & 8 & 71 & 11 & & & 24 & 4 & & \\
\hline $\mathrm{H} \delta$ & 4101 & 20 & 6 & & & 24 & 12 & 20 & 4 & & \\
\hline $\mathrm{H} \gamma$ & 4340 & 41 & 7 & 55 & 36 & 52 & 8 & 47 & 6 & 41 & 7 \\
\hline [O III] & 4363 & & & & & & & & & & \\
\hline He I & 4471 & & & & & & & 5 & 3 & & \\
\hline $\mathrm{He}$ II & 4686 & & & 39 & 9 & & & & & & \\
\hline $\mathrm{H} \beta$ & 4861 & 100 & 0 & 100 & 0 & 100 & 0 & 100 & 0 & 100 & 0 \\
\hline
\end{tabular}


Table 12. continued.

\begin{tabular}{|c|c|c|c|c|c|c|c|c|c|c|c|}
\hline [O III] & 4959 & 343 & 23 & 602 & 58 & 176 & 13 & 218 & 12 & 284 & 25 \\
\hline [O III] & 5007 & 1046 & 68 & 1772 & 168 & 554 & 36 & 645 & 32 & 760 & 63 \\
\hline $\mathrm{He} \mathrm{I}$ & 5876 & 24 & 6 & & & & & 6 & 2 & 28 & 10 \\
\hline [N II] & 6548 & & & & & 42 & 6 & & & & \\
\hline $\mathrm{H} \alpha$ & 6562 & 281 & 19 & 281 & 28 & 282 & 19 & 281 & 14 & 281 & 24 \\
\hline$[\mathrm{N} \mathrm{II}]$ & 6583 & 60 & 6 & 140 & 16 & 131 & 10 & 114 & 6 & 15 & 6 \\
\hline$[\mathrm{S}$ II $]$ & 6716 & 15 & 4 & & & & & 5 & 2 & & \\
\hline [S II] & 6730 & 20 & 6 & & & & & 9 & 3 & 21 & 8 \\
\hline [Ar III] & 7133 & 16 & 4 & & & 22 & 5 & 22 & 4 & & \\
\hline [O II] & 7325 & & & & & & & & & 28 & 5 \\
\hline $\mathrm{c}$ & & 0.37 & 0.09 & 0.69 & 0.14 & 0.61 & 0.09 & 0.35 & 0.07 & 0.30 & 0.12 \\
\hline $\log F(\mathrm{H} \beta)$ & & -16.19 & 0.03 & -16.09 & 0.04 & -16.01 & 0.03 & -16.08 & 0.02 & -16.32 & 0.04 \\
\hline \multirow[t]{2}{*}{$m_{5007 \mathrm{~A}}$} & & 24.19 & 0.07 & 23.37 & 0.10 & 24.41 & 0.07 & 24.43 & 0.05 & 24.86 & 0.09 \\
\hline & & \multicolumn{2}{|c|}{$\begin{array}{c}\mathrm{F} 42 \# 7 \\
0519 \\
(-253.4,-81.8) \\
\end{array}$} & \multicolumn{2}{|c|}{$\begin{array}{c}\mathrm{F} 42 \# 8 \\
4217 \\
(-229.7,-119.5) \\
\end{array}$} & \multicolumn{2}{|c|}{$\begin{array}{c}\text { F42 \#9 } \\
4244 \\
(-209.0,-72.3) \\
\end{array}$} & \multicolumn{2}{|c|}{$\begin{array}{c}\mathrm{F} 42 \# 10 \\
4211 \\
(-179.6,-119.8) \\
\end{array}$} & \multicolumn{2}{|c|}{$\begin{array}{c}\mathrm{F} 42 \# 11 \\
0509 \\
(-165.4,-52.4) \\
\end{array}$} \\
\hline Species & $\lambda(\AA)$ & $F_{\text {Dered }}$ & \pm & $F_{\text {Dered }}$ & \pm & $F_{\text {Dered }}$ & \pm & $F_{\text {Dered }}$ & \pm & $F_{\text {Dered }}$ & \pm \\
\hline$[\mathrm{O}$ II] & 3727 & & & 52 & 9 & 16 & 11 & 69 & 6 & & \\
\hline$[\mathrm{Ne}$ III $]$ & 3868 & 105 & 12 & 99 & 10 & 87 & 13 & 76 & 6 & 55 & 9 \\
\hline$[\mathrm{Ne} \mathrm{III}]+\mathrm{H} \epsilon$ & 3970 & 46 & 9 & 38 & 9 & 34 & 13 & 24 & 5 & & \\
\hline $\mathrm{H} \delta$ & 4101 & 25 & 7 & 22 & 7 & & & 28 & 7 & 22 & 6 \\
\hline $\mathrm{H} \gamma$ & 4340 & 47 & 8 & 44 & 8 & 59 & 38 & 52 & 9 & 48 & 11 \\
\hline [O III] & 4363 & & & & & & & 12 & 7 & & \\
\hline He I & 4471 & & & & & & & & & & \\
\hline $\mathrm{He}$ II & 4686 & 24 & 8 & & & & & 21 & 5 & & \\
\hline $\mathrm{H} \beta$ & 4861 & 100 & 0 & 100 & 0 & 100 & 0 & 100 & 0 & 100 & 0 \\
\hline [O III] & 4959 & 498 & 41 & 421 & 30 & 330 & 29 & 262 & 14 & 306 & 26 \\
\hline [O III] & 5007 & 1460 & 116 & 1268 & 87 & 1036 & 86 & 786 & 39 & 944 & 76 \\
\hline He I & 5876 & & & & & 23 & 9 & & & & \\
\hline$[\mathrm{N} \mathrm{II}]$ & 6548 & & & & & & & 37 & 13 & 89 & 18 \\
\hline $\mathrm{H} \alpha$ & 6562 & 282 & 23 & 283 & 21 & 282 & 25 & 282 & 15 & 281 & 25 \\
\hline$[\mathrm{N} \mathrm{II}]$ & 6583 & 97 & 11 & 116 & 10 & 80 & 10 & 175 & 10 & 170 & 16 \\
\hline $\mathrm{He} \mathrm{I}$ & 6678 & & & & & & & 9 & 3 & & \\
\hline [S II $]$ & 6716 & & & & & & & 14 & 3 & & \\
\hline$[\mathrm{S} \mathrm{II}]$ & 6730 & & & & & & & 23 & 4 & 12 & 5 \\
\hline [Ar III] & 7133 & & & & & 30 & 7 & 17 & 3 & 59 & 17 \\
\hline$[\mathrm{O} \mathrm{II}]$ & 7325 & & & & & 44 & 19 & & & & \\
\hline $\mathrm{c}$ & & 0.52 & 0.12 & 0.45 & 0.10 & 0.53 & 0.13 & 0.64 & 0.07 & 0.39 & 0.12 \\
\hline $\log F(\mathrm{H} \beta)$ & & -16.11 & 0.03 & -16.15 & 0.03 & -16.17 & 0.04 & -15.74 & 0.02 & -16.20 & 0.04 \\
\hline \multirow[t]{2}{*}{$m_{5007 \mathrm{~A}}$} & & 23.61 & 0.09 & 23.88 & 0.08 & 24.15 & 0.09 & 23.36 & 0.05 & 24.31 & 0.09 \\
\hline & & \multicolumn{2}{|c|}{$\begin{array}{c}\mathrm{F} 42 \# 12 \mathrm{~b} \\
4214 \\
(-135.0,-115.2) \\
\end{array}$} & \multicolumn{2}{|c|}{$\begin{array}{c}\mathrm{F} 42 \# 13 \\
4248 \\
(-115.2,-103.1)\end{array}$} & \multicolumn{2}{|c|}{$\begin{array}{c}\mathrm{F} 42 \# 14 \mathrm{~b} \\
4210 \\
(-99.8,-73.0) \\
\end{array}$} & \multicolumn{2}{|c|}{$\begin{array}{c}\mathrm{F} 42 \# 15 \mathrm{~b} \\
4245 \\
(-68.7,-65.2) \\
\end{array}$} & \multicolumn{2}{|c|}{$\begin{array}{c}\text { F42 \#16 } \\
4207 \\
(-48.4,-102.4)\end{array}$} \\
\hline Species & $\lambda(\AA)$ & $F_{\text {Dered }}$ & \pm & $F_{\text {Dered }}$ & \pm & $F_{\text {Dered }}$ & \pm & $F_{\text {Dered }}$ & \pm & $F_{\text {Dered }}$ & \pm \\
\hline [O II] & 3727 & & & 91 & 52 & 78 & 60 & & & 83 & 18 \\
\hline [Ne III] & 3868 & 174 & 22 & 230 & 122 & 240 & 48 & 59 & 13 & 125 & 20 \\
\hline$[\mathrm{Ne} \mathrm{III}]+\mathrm{H} \epsilon$ & 3970 & 94 & 14 & 78 & 46 & & & & & 42 & 12 \\
\hline $\mathrm{H} \delta$ & 4101 & & & & & & & & & & \\
\hline $\mathrm{H} \gamma$ & 4340 & 51 & 15 & & & & & 37 & 20 & 50 & 17 \\
\hline [O III] & 4363 & & & & & & & & & 21 & 9 \\
\hline He I & 4471 & & & & & & & & & & \\
\hline $\mathrm{He}$ II & 4686 & 15 & 13 & 33 & 26 & 62 & 32 & & & & \\
\hline $\mathrm{H} \beta$ & 4861 & 100 & 0 & 100 & 0 & 100 & 0 & 100 & 0 & 100 & 0 \\
\hline [O III] & 4959 & 545 & 55 & 660 & 341 & 763 & 135 & 261 & 32 & 561 & 73 \\
\hline [O III] & 5007 & 1637 & 161 & 2046 & 1053 & 2290 & 401 & 831 & 96 & 1688 & 216 \\
\hline $\mathrm{He} \mathrm{I}$ & 5876 & & & & & & & & & & \\
\hline$[\mathrm{N} \mathrm{II}]$ & 6548 & & & & & & & & & 46 & 12 \\
\hline $\mathrm{H} \alpha$ & 6562 & 282 & 29 & 282 & 146 & 281 & 51 & 281 & 33 & 281 & 38 \\
\hline$[\mathrm{N} \mathrm{II}]$ & 6583 & 110 & 13 & 136 & 72 & 97 & 21 & & & 139 & 22 \\
\hline He I & 6678 & & & & & & & & & & \\
\hline [S II] & 6716 & & & & & & & & & & \\
\hline
\end{tabular}


J. R. Walsh et al.: Abundance distribution in NGC 5128 from PNe

Table 12. continued.

\begin{tabular}{|c|c|c|c|c|c|c|c|c|c|c|c|}
\hline $\begin{array}{l}{[\mathrm{S} \mathrm{II}]} \\
{[\mathrm{Ar} \text { III }]} \\
{[\mathrm{O} \mathrm{III}]}\end{array}$ & $\begin{array}{l}6730 \\
7133 \\
7325\end{array}$ & & & & & & & 22 & 6 & 29 & 12 \\
\hline $\mathrm{c}$ & & 0.81 & 0.14 & 0.82 & 0.72 & 0.78 & 0.25 & 0.60 & 0.17 & 0.44 & 0.19 \\
\hline $\log F(\mathrm{H} \beta)$ & & -15.92 & 0.04 & -16.28 & 0.22 & -16.10 & 0.08 & -16.04 & 0.05 & -16.21 & 0.06 \\
\hline \multirow[t]{2}{*}{$m_{5007 \mathrm{~A}}$} & & 23.01 & 0.11 & 23.69 & 0.56 & 23.11 & 0.19 & 24.06 & 0.13 & 23.71 & 0.14 \\
\hline & & \multicolumn{4}{|c|}{$\begin{array}{cc}\text { F42 \#17 } & \text { F42 \#18 } \\
4264 & 4212 \\
(-13.9,-166.9) & (-5.9,-115.2) \\
\end{array}$} & & & & & & \\
\hline Species & $\lambda(\AA)$ & $F_{\text {Dered }}$ & \pm & $F_{\text {Dered }}$ & \pm & & & & & & \\
\hline$[\mathrm{O} \mathrm{II}]$ & 3727 & \multirow{8}{*}{43} & \multirow{7}{*}{10} & \multirow{4}{*}{67} & \multirow{4}{*}{14} & & & & & & \\
\hline$[\mathrm{Ne} \mathrm{III}]$ & 3868 & & & & & & & & & & \\
\hline$[\mathrm{Ne} \mathrm{III}]+\mathrm{H} \epsilon$ & 3970 & & & & & & & & & & \\
\hline $\mathrm{H} \delta$ & 4101 & & & & & & & & & & \\
\hline $\mathrm{H} \gamma$ & 4340 & & & \multirow[t]{3}{*}{61} & \multirow[t]{3}{*}{14} & & & & & & \\
\hline [O III] & 4363 & & & & & & & & & & \\
\hline He I & 4471 & & & & & & & & & & \\
\hline $\mathrm{He}$ II & 4686 & & & 19 & 15 & & & & & & \\
\hline $\mathrm{H} \beta$ & 4861 & 100 & 0 & 100 & 0 & & & & & & \\
\hline [O III] & 4959 & 201 & 16 & 524 & 58 & & & & & & \\
\hline [O III] & 5007 & 571 & 39 & 1490 & 161 & & & & & & \\
\hline $\mathrm{He} \mathrm{I}$ & 5876 & & & & & & & & & & \\
\hline$[\mathrm{N} \mathrm{II}]$ & 6548 & 29 & 6 & & & & & & & & \\
\hline $\mathrm{H} \alpha$ & 6562 & 281 & 20 & 283 & 32 & & & & & & \\
\hline$[\mathrm{N} \mathrm{III}]$ & 6583 & 108 & 10 & 33 & 8 & & & & & & \\
\hline He I & 6678 & & & & & & & & & & \\
\hline [S II] & 6716 & & & & & & & & & & \\
\hline [S II $]$ & 6730 & & & & & & & & & & \\
\hline [Ar III] & 7133 & & & & & & & & & & \\
\hline [O II] & 7325 & & & & & & & & & & \\
\hline $\mathrm{c}$ & & 0.79 & 0.10 & 1.01 & 0.16 & & & & & & \\
\hline $\log F(\mathrm{H} \beta)$ & & -15.82 & 0.03 & -15.64 & 0.05 & & & & & & \\
\hline \multirow[t]{2}{*}{$m_{5007 \mathrm{~A}}$} & & 23.91 & 0.07 & 22.41 & 0.12 & & & & & & \\
\hline & & \multicolumn{2}{|c|}{$\begin{array}{c}\text { F34 \#1 } \\
2606 \\
(-497.0,-921.7)\end{array}$} & \multicolumn{2}{|c|}{$\begin{array}{c}\text { F34 \#2 } \\
2603 \\
(-548.8,-902.6)\end{array}$} & \multicolumn{2}{|c|}{$\begin{array}{c}\text { F34 \#4 } \\
2607 \\
-446.9,-860.0) \\
\end{array}$} & $\begin{array}{r}\mathrm{F} 3 \\
34 \\
(-433.1 \\
\end{array}$ & $790.8)$ & $\begin{array}{r}\mathrm{F} 3 \\
3 \\
(-409 . \\
\end{array}$ & $693.3)$ \\
\hline Species & $\lambda(\AA)$ & $F_{\text {Dered }}$ & \pm & $F_{\text {Dered }}$ & \pm & $F_{\text {Dered }}$ & \pm & $F_{\text {Dered }}$ & \pm & $F_{\text {Dered }}$ & \pm \\
\hline [O II] & 3727 & 86 & 9 & 106 & 12 & 71 & 13 & 80 & 41 & 41 & 15 \\
\hline [Ne III] & 3868 & 93 & 6 & 71 & 7 & 70 & 7 & 137 & 8 & 127 & 15 \\
\hline$[\mathrm{Ne} \mathrm{III}]+\mathrm{H} \epsilon$ & 3970 & 31 & 5 & & & 48 & 8 & 53 & 7 & 36 & 9 \\
\hline $\mathrm{H} \delta$ & 4101 & 28 & 5 & 23 & 6 & 31 & 6 & 33 & 7 & 31 & 7 \\
\hline $\mathrm{H} \gamma$ & 4340 & 41 & 4 & 56 & 7 & 56 & 7 & 52 & 7 & 57 & 10 \\
\hline [O III] & 4363 & & & 11 & 5 & & & 9 & 3 & 21 & 11 \\
\hline $\mathrm{He} \mathrm{I}$ & 4471 & & & 20 & 5 & & & 10 & 3 & 20 & 8 \\
\hline He II & 4686 & 2 & 2 & 8 & 4 & & & $\mathrm{t}$ & & 51 & 10 \\
\hline $\mathrm{H} \beta$ & 4861 & 100 & 0 & 100 & 0 & 100 & 0 & 100 & 0 & 100 & 0 \\
\hline [O III] & 4959 & 393 & 15 & 249 & 13 & 307 & 15 & 517 & 21 & 514 & 43 \\
\hline [O III] & 5007 & 1164 & 41 & 729 & 34 & 922 & 43 & 1512 & 59 & 1505 & 124 \\
\hline $\mathrm{He} \mathrm{I}$ & 5876 & & & 21 & 6 & & & & & & \\
\hline [N II] & 6548 & 23 & 6 & & & & & & & & \\
\hline $\mathrm{H} \alpha$ & 6562 & 283 & 12 & 281 & 15 & 281 & 15 & 282 & 12 & 282 & 28 \\
\hline$[\mathrm{N} \mathrm{II}]$ & 6583 & 104 & 6 & 57 & 6 & 93 & 7 & 73 & 6 & 18 & 8 \\
\hline $\mathrm{He} \mathrm{I}$ & 6678 & & & 13 & 3 & & & & & 5 & 5 \\
\hline [S II] & 6716 & & & & & & & & & & \\
\hline [S II] & 6730 & & & & & & & & & & \\
\hline [Ar III] & 7133 & 4 & 3 & & & 21 & 5 & 21 & 4 & & \\
\hline [O II] & 7325 & 4 & 3 & & & 17 & 9 & & & & \\
\hline $\mathrm{c}$ & & 0.52 & 0.06 & 0.88 & 0.07 & 0.67 & 0.08 & 0.64 & 0.06 & 0.56 & 0.14 \\
\hline $\log F(\mathrm{H} \beta)$ & & -16.04 & 0.02 & -15.82 & 0.03 & -16.02 & 0.03 & -15.95 & 0.03 & -16.38 & 0.05 \\
\hline$m_{5007 \mathrm{~A}}$ & & 23.70 & 0.04 & 23.66 & 0.05 & 23.89 & 0.05 & 23.18 & 0.04 & 24.27 & 0.09 \\
\hline & & $\overline{\text { F34 }}$ & & F3 & & & & & & & \\
\hline
\end{tabular}


A\&A 544, A70 (2012)

Table 12. continued.

\begin{tabular}{|c|c|c|c|c|c|c|c|c|c|c|}
\hline \multirow[b]{2}{*}{ Species } & \multirow[b]{2}{*}{$\lambda(\AA)$} & \multicolumn{2}{|c|}{$\begin{array}{c}3405 \\
(-424.4,-682.1)\end{array}$} & \multicolumn{2}{|c|}{$\begin{array}{c}3505 \\
(-458,3,-622.8)\end{array}$} & \multicolumn{2}{|c|}{$\begin{array}{c}3504 \\
(-490.7,-611.0)\end{array}$} & \multicolumn{2}{|c|}{$\begin{array}{c}3506 \\
(-434.5,-592.1)\end{array}$} & \\
\hline & & $F_{\text {Dered }}$ & \pm & $F_{\text {Dered }}$ & \pm & $F_{\text {Dered }}$ & \pm & $F_{\text {Dered }}$ & \pm & \\
\hline [O II] & 3727 & 53 & 17 & 48 & 6 & & & 203 & 24 & \\
\hline [Ne III] & 3868 & 37 & 7 & 82 & 6 & 135 & 14 & 157 & 19 & \\
\hline$[\mathrm{Ne} \mathrm{III}]+\mathrm{H} \epsilon$ & 3970 & & & 36 & 5 & & & 64 & 13 & \\
\hline $\mathrm{H} \delta$ & 4101 & & & 18 & 13 & 26 & 8 & & & \\
\hline $\mathrm{H} \gamma$ & 4340 & 37 & 8 & 53 & 6 & 55 & 8 & 55 & 15 & \\
\hline [O III] & 4363 & & & 8 & 3 & 16 & 6 & 30 & 10 & \\
\hline $\mathrm{He} \mathrm{I}$ & 4471 & & & 5 & 3 & & & & & \\
\hline $\mathrm{He}$ II & 4686 & 46 & 9 & & & 23 & 16 & 50 & 13 & \\
\hline $\mathrm{H} \beta$ & 4861 & 100 & 0 & 100 & 0 & 100 & 0 & 100 & 0 & \\
\hline [O III] & 4959 & 473 & 45 & 394 & 18 & 438 & 29 & 513 & 48 & \\
\hline [O III] & 5007 & 1405 & 130 & 1153 & 51 & 1274 & 82 & 1560 & 143 & \\
\hline He I & 5876 & 45 & 23 & & & & & & & \\
\hline$[\mathrm{N} \mathrm{II}]$ & 6548 & & & & & & & 54 & 8 & \\
\hline $\mathrm{H} \alpha$ & 6562 & 282 & 26 & 281 & 14 & 282 & 20 & 281 & 29 & \\
\hline$[\mathrm{N} \mathrm{II}]$ & 6583 & 18 & 7 & 43 & 6 & 25 & 6 & 255 & 27 & \\
\hline $\mathrm{He} \mathrm{I}$ & 6678 & & & & & & & & & \\
\hline [S II] & 6716 & & & & & & & & & \\
\hline [S II] & 6730 & & & & & & & 21 & 13 & \\
\hline [Ar III] & 7133 & & & & & & & & & \\
\hline [O II] & 7325 & 48 & 22 & & & & & & & \\
\hline $\mathrm{c}$ & & 0.18 & 0.13 & 0.42 & 0.07 & 0.73 & 0.10 & 0.68 & 0.15 & \\
\hline $\log F(\mathrm{H} \beta)$ & & -16.81 & 0.05 & -16.22 & 0.03 & -16.10 & 0.04 & -16.36 & 0.04 & \\
\hline$m_{5007 \mathrm{~A}}$ & & 25.41 & 0.10 & 24.15 & 0.05 & 23.73 & 0.07 & 24.17 & 0.10 & \\
\hline
\end{tabular}

